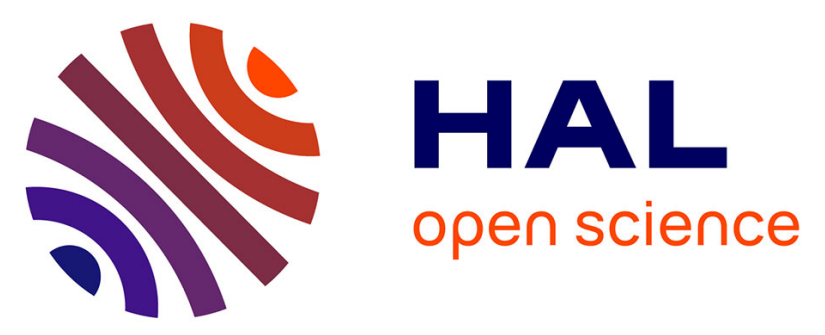

\title{
A unified variational framework for the space discontinuous Galerkin method for elastic wave propagation in anisotropic and piecewise homogeneous media
}

\author{
B. Tie, A.-S. Mouronval, D. Nguyen, L. Series, D. Aubry
}

\section{To cite this version:}

B. Tie, A.-S. Mouronval, D. Nguyen, L. Series, D. Aubry. A unified variational framework for the space discontinuous Galerkin method for elastic wave propagation in anisotropic and piecewise homogeneous media. Computer Methods in Applied Mechanics and Engineering, 2018, 338, pp.299 - 332. 10.1016/j.cma.2018.04.018 . hal-01832541

\section{HAL Id: hal-01832541 \\ https://hal.science/hal-01832541}

Submitted on 5 Oct 2020

HAL is a multi-disciplinary open access archive for the deposit and dissemination of scientific research documents, whether they are published or not. The documents may come from teaching and research institutions in France or abroad, or from public or private research centers.
L'archive ouverte pluridisciplinaire HAL, est destinée au dépôt et à la diffusion de documents scientifiques de niveau recherche, publiés ou non, émanant des établissements d'enseignement et de recherche français ou étrangers, des laboratoires publics ou privés. 


\title{
A unified variational framework for the space discontinuous Galerkin method for elastic wave propagation in anisotropic and piecewise homogeneous media
}

\author{
B. Tie ${ }^{\mathrm{a}, *}$, A.-S. Mouronval ${ }^{\mathrm{a}}$, V.-D. Nguyen ${ }^{\mathrm{a}}$, L. Series ${ }^{\mathrm{b}}$, D. Aubry ${ }^{\mathrm{a}}$ \\ ${ }^{a}$ Laboratory MSSMat (UMR8579-CNRS), CentraleSupélec, Université Paris-Saclay, 8-10 \\ rue Joliot-Curie, 91190 Gif-sur-Yvette, France \\ ${ }^{b}$ Laboratory MICS, CentraleSupélec, Université Paris-Saclay, 8-10 rue Joliot-Curie, 91190 \\ Gif-sur-Yvette, France
}

\begin{abstract}
We present a unified multidimensional variational framework for the space discontinuous Galerkin method for elastic wave propagation in anisotropic and piecewise homogeneous media. Based on an elastic wave oriented formulation and using a tensorial formalism, the proposed framework allows a better understanding of the physical meaning of the terms involved in the discontinuous Galerkin method. The unified variational framework is written for first-order velocity-stress wave equations. An uncoupled upwind numerical flux and two coupled upwind numerical fluxes using respectively the Voigt and the Reuss averages of elastic moduli are defined. Two numerical fluxes that are exact solutions of the Riemann problem on physical interfaces are also developed and analyzed in the $1 \mathrm{D}$ case. The implemented solvers are then applied to different elastic media, especially to polycrystalline materials that present a particular case of piecewise homogeneous media. The use of the three upwind numerical fluxes, which only solve approximately the Riemann problem at element interfaces, is investigated.
\end{abstract}

Keywords: Space discontinuous Galerkin method, elastic wave propagation, anisotropy, piecewise homogeneous medium, polycrystalline materials

\section{Introduction}

Numerical modeling of elastic wave propagation is a classical problem and can be performed reliably and efficiently in a large number of cases today. How-

\footnotetext{
* Corresponding author

Email addresses: bing.tie@centralesupelec.fr (B. Tie), anne-sophie.mouronval@centralesupelec.fr (A.-S. Mouronval), dang.1032170@gmail.com (V.-D. Nguyen), laurent.series@centralesupelec.fr (L. Series), denis.aubry@centralesupelec.fr (D. Aubry)
}

Preprint submitted to Comput. Methods Appl. Mech. Engrg. 
ever, the accurate and efficient simulation of elastic waves propagation in large 5 heterogeneous media still remains a challenging task, especially when high frequency ranges are involved and/or when the geometries of the whole domain or of the interior physical interfaces of heterogeneities are complicated. Therefore, in the past two decades, many research works have been carried on the space discontinuous Galerkin $(\mathrm{dG})$ methods that combine the advantages of finite el-

10 ement methods in the space with the possibilities of developing high-order time integration schemes and massively parallel solvers [1 8 .

When space dG methods are applied the first-order elastic wave equations, the Jacobian matrices of the hyperbolic system, if expressed in matrix form, are very complicated due to the fourth-order Hooke elastic tensor even in the 15 isotropic case and are different in $2 \mathrm{D}$ and $3 \mathrm{D}$ cases 2,3 . As a consequence, the physical meaning of the terms involved in the variational dG formulations, more particularly those concerned by numerical fluxes, are hidden and it becomes difficult to develop and analyze numerical fluxes in more complex cases of anisotropic and piecewise homogeneous media. The aim of the present work

20 is to address these concerns by defining a unified and elastic wave oriented variational framework for the first-order velocity-stress wave equations [2, 5, 9] in the multidimensional and general - anisotropic and piecewise homogeneous - case. An elastic wave oriented eigen analysis using a tensorial formalism is presented and allows a simple, compact and intrinsic expression of the Jaco25 bian operator of the first-order hyperbolic system in terms of its eigenvalues and eigenmodes. Hence definition of a unified variational framework is possible whatever the space dimension of propagation media and the characteristics of the elastic tensor. An equivalent eigen analysis is developed in [5], however, it is formulated within matrix forms and is quite complicated.

30 Three upwind numerical fluxes are considered in the present work. Firstly, the upwind numerical flux proposed by Käser et al. 2], which uses only material properties from the interior of elements and from one side in the case with discontinuous material properties, is merely modified in order to use material properties from elements on both sides of physical interfaces. This flux is called 35 "uncoupled" upwind flux herein. Secondly, to reinforce the coupling between the discontinuous material properties on both sides of a physical interface, a natural choice is to use their averages to define numerical fluxes. Both arithmetic and harmonic averages are considered and lead to two other numerical fluxes, called "coupled" upwind fluxes herein. Without discontinuous heterogeneities, 40 all these numerical fluxes are identical and exactly solve the Riemann problem on element interfaces, which is no longer true otherwise. In the general case with discontinuous heterogeneities, the use of all the three simplified fluxes needs to be investigated for verification.

Even though space dG methods can be combined successfully with high order 45 time integration schemes, such as the ADER (arbitrary high order derivatives) approach [2, 10], only a fourth-order Runge-Kutta method is used in the present work as the time domain solving is not the object of the present study. The obtained dG solver is explicit with a global mass matrix composed by completely uncoupled elementary mass matrices. Consequently, its parallelization based on 
MPI is straightforward.

The implemented solvers are firstly applied to both 1D and 2D media with only one physical interface. Accuracy of the three numerical fluxes to describe wave reflection, transmission and conversion phenomena is considered. Furthermore, to analyze the observed characteristics of both coupled upwind fluxes,

55 especially why in some cases the harmonic average of the Hooke tensor does not behave as good as the arithmetic one, unlike observations made in other applications (e.g. [11]), two numerical fluxes that are exact solutions of the Riemann problem defined on physical interfaces are developed and analyzed in the $1 \mathrm{D}$ case.

60 As numerical applications, we are interested in numerical modeling of ultrasound propagation in polycrystalline materials within the context of nondestructive testing. All the developed solvers are finally applied to single-phase and untextured polycrystalline materials composed of a large number of elliptic grains. Limitations of all the three simplified upwind numerical fluxes are investigated.

The paper is organized as follows. In Section 2, the first-order velocitystress hyperbolic system of elastic wave equations is introduced and an elastic wave oriented eigen analysis is given. Section 3 presents a unified variational framework within which the space dG method is introduced. The different nu70 merical fluxes considered in the present work are defined. The element-wise and global semi discretized formulations are given and the time domain integration using the algorithm of the fourth-stage fourth-order Runge-Kutta scheme is introduced. Section 4 presents numerical results obtained successively in 1D bimaterial, 2D bimaterial and 2D polycrystalline cases. Concluding remarks are given in Section 5 .

\section{First-order elastic wave governing equations}

The governing equations of elastic wave propagation are given. They are formulated as a first-order system with the velocity and stress fields as primary unknowns and keep using the tensorial formalism for the fourth-order elastic

80 Hooke tensor. Compared to the usual generic form of the first-order hyperbolic system in which the Hooke tensor is expressed in matrix forms (e.g. [3, 6, 7, 9, ), we show that the proposed formulation allows defining the Jacobian matrices and analyzing the eigen characteristic of the hyperbolic system in a unified and elastic wave oriented way.

We consider the wave propagation in an elastic medium $\Omega \subset \mathbb{R}^{d}$ of space dimension $d(d=1,2,3)$ and in a time interval $[0, T]$. It is governed by the following first-order equations in terms of the velocity field $\boldsymbol{v}(\boldsymbol{x}, t)=\partial_{t} \boldsymbol{u}(\boldsymbol{x}, t)$, $\boldsymbol{u}(\boldsymbol{x}, t)$ being the displacement field, and the second-order Cauchy stress tensor $\boldsymbol{\sigma}(\boldsymbol{x}, t): \forall(\boldsymbol{x}, t) \in \Omega \times] 0, T[$

$$
\begin{aligned}
& \partial_{t} \boldsymbol{v}-\rho^{-1} \mathbf{D i v}_{x} \boldsymbol{\sigma}-\rho^{-1} \boldsymbol{f}=\mathbf{0} \\
& \partial_{t} \boldsymbol{\sigma}-\boldsymbol{C}: \boldsymbol{\varepsilon}(\boldsymbol{v})=\mathbf{0}
\end{aligned}
$$


with $\rho$ the density, $\boldsymbol{f}$ the body force and $\boldsymbol{C}$ the fourth-order elasticity Hooke tensor. Herein, all the vectors and tensors are denoted using bold letters. In the following the body force $f$ will be omitted without loss of generality of the purpose of the present work.

In (1), the first equation expresses the elastodynamic equilibrium and the second equation is the time derivative of the Hooke's law of elasticity:

$$
\boldsymbol{\sigma}=\boldsymbol{C}: \varepsilon(\boldsymbol{u})
$$

where":" denotes the usual double dot product between two tensors defined as $(\boldsymbol{C}: \boldsymbol{\varepsilon})_{i j}=C_{i j k l} \varepsilon_{k l}$. We note that herein the Einstein summation convention is systematically used. According to the definition of the second-order infinitesimal strain tensor $\varepsilon$, we have:

$$
\boldsymbol{\varepsilon}(\boldsymbol{v})=\frac{1}{2}\left(\mathbf{D}_{x} \boldsymbol{v}+\mathbf{D}_{x}^{T} \boldsymbol{v}\right)
$$

where $\boldsymbol{A}^{T}$ denotes the transpose of $\boldsymbol{A}$. It is useful to recall the usual space gradient and divergence operators in the orthonormal basis $\left(\boldsymbol{e}_{i}\right)_{i=1, \ldots, d}$ :

$$
\begin{aligned}
\mathbf{D}_{x} \boldsymbol{v} & =\frac{\partial \boldsymbol{v}}{\partial x_{i}} \otimes \boldsymbol{e}_{i} \\
\operatorname{Div}_{x} \boldsymbol{\sigma} & =\frac{\partial \boldsymbol{\sigma}}{\partial x_{i}} \cdot \boldsymbol{e}_{i}
\end{aligned}
$$

where " $\otimes$ " denotes the usual tensor product between two vectors: $(\boldsymbol{a} \otimes \boldsymbol{b})_{i j}=$ $a_{i} b_{j}$ and "." the usual dot product between a tensor and a vector: $(\boldsymbol{A} \cdot \boldsymbol{a})_{i}=$ $A_{i j} a_{j}$. In the following, the symmetrized tensor product denoted by " $\otimes_{s}$ " is also used: $\boldsymbol{a} \otimes_{s} \boldsymbol{b}=(\boldsymbol{a} \otimes \boldsymbol{b}+\boldsymbol{b} \otimes \boldsymbol{a}) / 2$.

\subsection{A tensorial compact form for the first-order wave equations}

Now, we propose to rewrite the first-order velocity-stress system (1) into a tensorial compact form. To do that we define a generalized unknown $\boldsymbol{U}(\boldsymbol{x}, t)=$ $(\boldsymbol{v}(\boldsymbol{x}, t) \boldsymbol{\sigma}(\boldsymbol{x}, t))^{T}$, which is a field in $\mathbb{R}^{d} \times \mathbb{R}^{d \times d_{s y m}}$ and defined over the open set $\Omega \times] 0, T\left[\mathbb{R}^{d \times d_{s y m}}\right.$ indicates that $\boldsymbol{\sigma}(\boldsymbol{x}, t)$ is a $d \times d$ symmetric second-order tensor field. By defining the following space derivation operator $\boldsymbol{A}^{\partial_{x}}$ :

$$
\boldsymbol{A}^{\partial_{x}}\left(\begin{array}{c}
\boldsymbol{v} \\
\boldsymbol{\sigma}
\end{array}\right)=\left(\begin{array}{c}
-\rho^{-1} \operatorname{Div}_{x} \boldsymbol{\sigma} \\
-\boldsymbol{C}: \varepsilon(\boldsymbol{v})
\end{array}\right)
$$

the first-order velocity-stress system (1) finally gets the following compact form:

$$
\partial_{t} \boldsymbol{U}+\boldsymbol{A}^{\partial_{x}}(\boldsymbol{U})=\mathbf{0}
$$

The dot product in the vectorial space $\mathbb{R}^{d} \times \mathbb{R}^{d \times d_{\text {sym }}}$ is defined in the fol115 lowing way: $\forall \boldsymbol{W}_{1}=\left(\boldsymbol{w}_{1} \boldsymbol{\tau}_{1}\right)^{T}$ and $\boldsymbol{W}_{2}=\left(\boldsymbol{w}_{2} \boldsymbol{\tau}_{2}\right)^{T} \in \mathbb{R}^{d} \times \mathbb{R}^{d \times d_{s y m}}$

$$
\boldsymbol{W}_{1} \cdot \boldsymbol{W}_{2}=\boldsymbol{w}_{1} \cdot \boldsymbol{w}_{2}+\boldsymbol{\tau}_{1}: \boldsymbol{\tau}_{2}
$$


According to (4), it is easy to show that the Jacobian operator $\boldsymbol{A}_{\boldsymbol{e}}$ in any spatial direction $\boldsymbol{e}$ of the first-order system (6) is defined as: $\forall \boldsymbol{W}=(\boldsymbol{w} \boldsymbol{\tau})^{T}$

$$
\boldsymbol{A}_{\boldsymbol{e}}(\boldsymbol{W})=\left(\begin{array}{c}
-\rho^{-1} \boldsymbol{\tau} \cdot \boldsymbol{e} \\
-\boldsymbol{C}:\left(\boldsymbol{e} \otimes_{s} \boldsymbol{w}\right)
\end{array}\right)
$$

Herein, the subscript index " $\boldsymbol{e}$ " indicates the dependency of $\boldsymbol{A}_{\boldsymbol{e}}$ on $\boldsymbol{e}$.

We remark that the first-order elastic wave formulation (6) can be written in the following way:

$$
\partial_{t} \boldsymbol{U}+\boldsymbol{A}_{\boldsymbol{e}_{i}}\left(\partial_{x_{i}} \boldsymbol{U}\right)=\mathbf{0}
$$

When the operators $\left(\boldsymbol{A}_{\boldsymbol{e}_{i}}\right)_{i=1, \ldots, d}$ are written in the matrix form in (9), the usual generic form of the first-order hyperbolic system used in the related literature (e.g. [3, 6, 7, 9]) can be recovered.

On the boundary of $\partial \Omega$ with outward unit normal vector $\boldsymbol{n}=n_{i} \boldsymbol{e}_{i}$, it can be shown (see also Section 3) that the flux operator $\boldsymbol{F}_{\boldsymbol{n}}$ associated with the first-order system (6) is in fact equal to $\boldsymbol{A}_{\boldsymbol{n}}=n_{i} \boldsymbol{A}_{\boldsymbol{e}_{i}}$ the Jacobian operator in the $\boldsymbol{n}$ direction:

$$
\boldsymbol{F}_{\boldsymbol{n}}(\boldsymbol{W})=\boldsymbol{A}_{\boldsymbol{n}}(\boldsymbol{W})=\left(\begin{array}{c}
\boldsymbol{F}_{\boldsymbol{n}, \boldsymbol{w}}(\boldsymbol{W}) \\
\boldsymbol{F}_{\boldsymbol{n}, \boldsymbol{\tau}}(\boldsymbol{W})
\end{array}\right)=\left(\begin{array}{c}
-\rho^{-1} \boldsymbol{\tau} \cdot \boldsymbol{n} \\
-\boldsymbol{C}:\left(\boldsymbol{n} \otimes_{s} \boldsymbol{w}\right)
\end{array}\right)
$$

In the following, the local orthonormal basis defined on $\partial \Omega$ will be denoted by $\left(\boldsymbol{n},\left\{\boldsymbol{t}_{\alpha}\right\}_{\alpha=1, \ldots, d-1}\right)$.

${ }_{130}$ To complete the definition of the first-order velocity-stress elastic wave problem, the following boundary and initial conditions are prescribed:

- Boundary conditions

$$
\begin{aligned}
& \left.\boldsymbol{F}_{\boldsymbol{n}, \boldsymbol{w}}(\boldsymbol{U})=-\rho^{-1} \boldsymbol{g} \quad, \quad \text { on } \partial \Omega_{N} \times\right] 0, T[ \\
& \left.\boldsymbol{F}_{\boldsymbol{n}, \boldsymbol{\tau}}(\boldsymbol{U})=-\boldsymbol{C}:\left(\boldsymbol{n} \otimes_{s} \partial_{t} \boldsymbol{u}_{D}\right) \quad, \quad \text { on } \partial \Omega_{D} \times\right] 0, T[
\end{aligned}
$$

where the first equation in 11 expresses the Neumann boundary conditions with imposed surface loadings $\boldsymbol{g}$ and the second one expresses the Dirichlet boundary conditions with prescribed displacements $\boldsymbol{u}_{D}$. We recall that the conditions $\partial \Omega_{N} \cup \partial \Omega_{D}=\partial \Omega$ and $\partial \Omega_{N} \cap \partial \Omega_{D}=\varnothing$ should be verified.

- Initial conditions

$$
\begin{aligned}
& \boldsymbol{\sigma}(\boldsymbol{x}, 0)=\boldsymbol{C}: \varepsilon\left(\boldsymbol{u}_{0}(\boldsymbol{x})\right) \quad, \quad \forall \boldsymbol{x} \in \Omega \\
& \boldsymbol{v}(\boldsymbol{x}, 0)=\boldsymbol{v}_{0}(\boldsymbol{x}) \quad, \forall \boldsymbol{x} \in \Omega
\end{aligned}
$$

\subsection{Elastic wave oriented eigen analysis}

Before considering the variational formulation of the first-order velocitystress system (6), it is worth analyzing its characteristic structure by performing the eigen analysis of its normal Jacobian operator $\boldsymbol{A}_{\boldsymbol{n}}$ defined in 10 . 
To find the $m=d+d(d+1) / 2$ eigenvalues $\left\{\lambda_{\boldsymbol{n}, k}\right\}_{k=1, \ldots, m}$ of $\boldsymbol{A}_{\boldsymbol{n}}$ and the corresponding right eigenvectors $\left\{\boldsymbol{R}_{\boldsymbol{n}, k}\right\}_{k=1, \ldots, m}=\left(\boldsymbol{w}_{\boldsymbol{n}, k}^{r} \boldsymbol{\tau}_{\boldsymbol{n}, k}^{r}\right)^{T}$, the following eigen problem is solved:

$$
\boldsymbol{A}_{\boldsymbol{n}}\left(\boldsymbol{R}_{\boldsymbol{n}, k}\right)=\lambda_{\boldsymbol{n}, k} \boldsymbol{R}_{\boldsymbol{n}, k} \text { or } \quad \begin{aligned}
-\rho^{-1} \boldsymbol{\tau}_{\boldsymbol{n}, k}^{r} \cdot \boldsymbol{n} & =\lambda_{\boldsymbol{n}, k} \boldsymbol{w}_{\boldsymbol{n}, k}^{r} \\
-\boldsymbol{C}:\left(\boldsymbol{n} \otimes_{s} \boldsymbol{w}_{\boldsymbol{n}, k}^{r}\right) & =\lambda_{\boldsymbol{n}, k} \boldsymbol{\tau}_{\boldsymbol{n}, k}^{r}
\end{aligned}
$$

It can be shown that the non-zero eigenvalues $\lambda_{n, k}$ of 13 satisfy the following eigen system of the Christoffel tensor $\boldsymbol{\Gamma}_{\boldsymbol{n}}$ :

$$
\boldsymbol{\Gamma}_{\boldsymbol{n}} \cdot \gamma_{\boldsymbol{n}, k}=\lambda_{\boldsymbol{n}, k}^{2} \gamma_{\boldsymbol{n}, k}
$$

with the definition of the Christoffel tensor $\boldsymbol{\Gamma}_{\boldsymbol{n}}$ recalled in the following:

$$
\begin{aligned}
\boldsymbol{\Gamma}_{\boldsymbol{n}} \cdot \boldsymbol{w} & =\rho^{-1}\left(\boldsymbol{C}:\left(\boldsymbol{n} \otimes_{s} \boldsymbol{w}\right)\right) \cdot \boldsymbol{n}, \forall \boldsymbol{w} \\
\left(\boldsymbol{\Gamma}_{\boldsymbol{n}}\right)_{i j} & =\rho^{-1} n_{k} C_{i k j l} n_{l}
\end{aligned}
$$

The eigenvalues and eigenvectors of $\boldsymbol{\Gamma}_{\boldsymbol{n}}$ are in fact the wave velocities and the corresponding waves modes in $\Omega$ characterized by $(\rho, \boldsymbol{C})$. Herein, the $d$ positive eigenvalues of $\boldsymbol{\Gamma}_{\boldsymbol{n}}$ are denoted by $c_{\boldsymbol{n}, q L}^{2}$ and $\left\{c_{\boldsymbol{n}, q T_{\alpha}}^{2}\right\}_{\alpha=1, \ldots, d-1},\left(c_{\boldsymbol{n}, q L}, c_{\boldsymbol{n}, q T_{\alpha}}\right)$ being respectively the velocity of quasi-longitudinal and quasi-transverse wave modes propagating in the $\boldsymbol{n}$ direction. The corresponding wave modes are denoted by the unit eigenvectors $\gamma_{\boldsymbol{n}, q L}$ and $\left\{\boldsymbol{\gamma}_{\boldsymbol{n}, q T_{\alpha}}\right\}_{\alpha=1, \ldots, d-1}$. For example, quasi-longitudinal wave mode and transverse wave modes. The subscript indices " $q L$ " and " $q T$ " respectively refer to quasi-longitudinal and quasi-transverse waves. We recall that in the general anisotropic case all the wave velocities and the waves modes depend on the space direction $\boldsymbol{n}$. The word "quasi" means that in the general anisotropic case we have neither pure longitudinal wave mode verifying $\gamma_{\boldsymbol{n}, q L} \| \boldsymbol{n}$ nor pure transverse waves modes verifying $\gamma_{\boldsymbol{n}, q T} \perp \boldsymbol{n}$, in contrast to the isotropic case.

To find the corresponding left eigenvectors $\left\{\boldsymbol{L}_{\boldsymbol{n}, k}\right\}_{k=1, \ldots, m}=\left(\boldsymbol{w}_{\boldsymbol{n}, k}^{l} \boldsymbol{\tau}_{\boldsymbol{n}, k}^{l}\right)^{T}$ of $\boldsymbol{A}_{\boldsymbol{n}}$, we consider its transpose $\boldsymbol{A}_{\boldsymbol{n}}^{T}$ defined by: $\forall \boldsymbol{W}=(\boldsymbol{w} \boldsymbol{\tau})^{T}$

$$
\boldsymbol{A}_{\boldsymbol{n}}^{T}(\boldsymbol{W})=\left(\begin{array}{c}
-(\boldsymbol{C}: \boldsymbol{\tau}) \cdot \boldsymbol{n} \\
-\rho^{-1} \boldsymbol{n} \otimes_{s} \boldsymbol{w}
\end{array}\right)
$$

It can be shown that the eigenvalues and the corresponding eigenvectors $\boldsymbol{w}_{\boldsymbol{n}, k}^{l}$ of (16) satisfy also the eigen equation of the Christoffel tensor (14), i.e. $\boldsymbol{w}_{\boldsymbol{n}, k}^{r}=$ $\boldsymbol{w}_{n, k}^{l}$, and both will be denoted by $\boldsymbol{w}_{\boldsymbol{n}, k}$ hereafter.

In summary, the $m$ eigenvalues of the normal Jacobian operator $\boldsymbol{A}_{\boldsymbol{n}}$ sorted from the lowest to the highest values are:

$$
\begin{aligned}
& \left\{\lambda_{\boldsymbol{n}, k}\right\}_{k=1, \ldots, m} \\
& =\{-c_{\boldsymbol{n}, q L},\left\{-c_{\boldsymbol{n}, q T_{\alpha}}\right\}_{\alpha=1, \ldots, d-1}, \underbrace{0, \ldots, 0}_{m-2 d \text { times }},\left\{c_{\boldsymbol{n}, q T_{\alpha}}\right\}_{\alpha=1, \ldots, d-1}, c_{\boldsymbol{n}, q L}\}
\end{aligned}
$$

The right and left eigenmodes corresponding to the non-zero eigenvalues, i.e. $170 \quad k=1, \ldots, d$ and $k=m-d+1, \ldots, m$, are:

$$
\boldsymbol{R}_{\boldsymbol{n}, k}=\left(\begin{array}{c}
\boldsymbol{w}_{\boldsymbol{n}, k} \\
-\lambda_{\boldsymbol{n}, k}^{-1} \boldsymbol{C}:\left(\boldsymbol{n} \otimes_{s} \boldsymbol{w}_{\boldsymbol{n}, k}\right)
\end{array}\right), \boldsymbol{L}_{\boldsymbol{n}, k}=\left(\begin{array}{c}
\boldsymbol{w}_{\boldsymbol{n}, k} \\
-\rho^{-1} \lambda_{\boldsymbol{n}, k}^{-1} \boldsymbol{n} \otimes_{s} \boldsymbol{w}_{\boldsymbol{n}, k}
\end{array}\right)
$$


with:

$$
\left\{\boldsymbol{w}_{\boldsymbol{n}, k}\right\}=\frac{1}{\sqrt{2}}\left\{\boldsymbol{\gamma}_{\boldsymbol{n}, q L},\left\{\boldsymbol{\gamma}_{\boldsymbol{n}, q T_{\alpha}}\right\}_{\alpha=1, \ldots, d-1},\left\{\boldsymbol{\gamma}_{\boldsymbol{n}, q T_{\alpha}}\right\}_{\alpha=1, \ldots, d-1}, \boldsymbol{\gamma}_{\boldsymbol{n}, q L}\right\}
$$

The right and left eigenmodes corresponding to the zero eigenvalues, i.e. $k=$ $d+1, \ldots, m-d$, are:

$$
\boldsymbol{R}_{\boldsymbol{n}, k}=\left(\begin{array}{c}
\mathbf{0} \\
\boldsymbol{\tau}_{\boldsymbol{n}, k}^{\perp \boldsymbol{n}}
\end{array}\right), \boldsymbol{L}_{\boldsymbol{n}, k}=\left(\begin{array}{c}
\mathbf{0} \\
\boldsymbol{\tau}_{\boldsymbol{n}, k}^{C, \perp \boldsymbol{n}}
\end{array}\right)
$$

with:

$$
\boldsymbol{\tau}_{\boldsymbol{n}, k}^{\boldsymbol{C}, \perp \boldsymbol{n}}=\frac{\boldsymbol{C}^{-1}: \boldsymbol{\tau}_{\boldsymbol{n}, k}^{\perp \boldsymbol{n}}}{\boldsymbol{\tau}_{\boldsymbol{n}, k}^{\perp \boldsymbol{n}}: \boldsymbol{C}^{-1}: \boldsymbol{\tau}_{\boldsymbol{n}, k}^{\perp \boldsymbol{n}}}
$$

175

where the superscript index " $\perp \boldsymbol{n}$ " of $\boldsymbol{\tau}^{\perp \boldsymbol{n}}$ refers to a "perpendicular to $\boldsymbol{n}$ " stress state, i.e. $\boldsymbol{\tau} \cdot \boldsymbol{n}=\mathbf{0}$ and the superscript index " $\boldsymbol{C}, \perp \boldsymbol{n}$ " of $\boldsymbol{\tau} \boldsymbol{C , \perp \boldsymbol { n }}$ refers to a strain state giving rise to a " $\perp \boldsymbol{n}$ " stress state, i.e. $\left(\boldsymbol{C}: \boldsymbol{\tau}^{\boldsymbol{C}, \perp \boldsymbol{n}}\right) \cdot \boldsymbol{n}=\mathbf{0}$. For example in the $3 \mathrm{D}$ case, $\boldsymbol{\tau}^{\perp n}$ is a plane stress state in the plane perpendicular to $\boldsymbol{n}$ and we can choose: $\boldsymbol{\tau}_{\boldsymbol{n}, 4}^{\perp \boldsymbol{n}}=\boldsymbol{t}_{1} \otimes \boldsymbol{t}_{1}, \boldsymbol{\tau}_{\boldsymbol{n}, 5}^{\perp \boldsymbol{n}}=\boldsymbol{t}_{1} \otimes_{s} \boldsymbol{t}_{2}$ and $\boldsymbol{\tau}_{\boldsymbol{n}, 6}^{\perp \boldsymbol{n}}=\boldsymbol{t}_{2} \otimes \boldsymbol{t}_{2}$.

It can be shown that the following orthonormality relation holds between the two right and left eigen bases:

$$
\boldsymbol{R}_{\boldsymbol{n}, i} \cdot \boldsymbol{L}_{\boldsymbol{n}, j}=\delta_{i j}, \forall i, j=1, \cdots, m
$$

where $\delta_{i j}$ denotes the usual Kronecker delta. Hence, the normal Jacobian operator $\boldsymbol{A}_{n}$ is decomposed using those two eigen bases as follows:

$$
\boldsymbol{A}_{\boldsymbol{n}}=\lambda_{\boldsymbol{n}, k}^{-} \boldsymbol{R}_{\boldsymbol{n}, k}^{-} \otimes \boldsymbol{L}_{\boldsymbol{n}, k}^{-}+\lambda_{\boldsymbol{n}, k}^{+} \boldsymbol{R}_{\boldsymbol{n}, k}^{+} \otimes \boldsymbol{L}_{\boldsymbol{n}, k}^{+}
$$

where $\left(\lambda_{\boldsymbol{n}, k}^{-}, \boldsymbol{R}_{\boldsymbol{n}, k}^{-}, \boldsymbol{L}_{\boldsymbol{n}, k}^{-}\right)_{k=q L,\left\{q T_{\alpha}\right\}}$ are the strictly negative eigenvalues and the corresponding right and left eigenvectors, and $\left(\lambda_{\boldsymbol{n}, k}^{+}, \boldsymbol{R}_{\boldsymbol{n}, k}^{+}, \boldsymbol{L}_{\boldsymbol{n}, k}^{+}\right)_{k=q L,\left\{q T_{\alpha}\right\}}$ the strictly positive ones.

We note that the decomposition (23) of the normal Jacobian operator $\boldsymbol{A}_{n}$ is exactly equivalent to the one presented in the related literature, e.g. 3. Indeed, even if all the eigenvalues including the zero are present in the decomposition developed in [3], by putting zeros in the diagonal of the diagonal matrix $\Gamma_{p s}$ of the eigenvalues, the final result is that all the eigenvectors corresponding to the zero eigenvalues are not included in the decomposition (see Eq.(15) in [3]). The decomposition (23) is more interesting as it is written in a completely intrinsic way independent from the definition of any global and local bases.

In summary, this elastic wave oriented eigen analysis allows expressing $\boldsymbol{A}_{n}$ in terms of wave modes and highlighting their role played in flux exchanges on the interface of two adjacent subdomains. For example, equations (20|21) show that the eigen wave modes corresponding to the zero eigenvalues are in fact the membrane wave modes in the plane perpendicular to $\boldsymbol{n}$, which means that both their propagation and polarization directions lay in that plane. The corresponding zero eigenvalues indicate that they cannot propagate in the $\boldsymbol{n}$ 
direction. In other words, as such a stress state results in a zero stress vector $\boldsymbol{\tau} \cdot \boldsymbol{n}=\mathbf{0}$, it makes no contribution to the equilibrium of the interface between two adjacent subdomains. This is coherent with the $\mathrm{dG}$ formulation presented in the next section, in which the wave modes 20 corresponding to zero eigenvalues are not considered in the flux terms exchanged between two finite elements having a $\boldsymbol{n}$-perpendicular interface.

Finally, it is interesting to give the example of the 3D isotropic case, for which the wave velocities are independent from $\boldsymbol{n}: c_{L}=\sqrt{(\lambda+2 \mu) / \rho}$ and $c_{T_{1}}=$ ${ }_{210} \quad c_{T_{2}}=\sqrt{\mu / \rho}$ with $(\lambda, \mu)$ the Lamé constants. The eigenvectors of the Christoffel tensor $\boldsymbol{\Gamma}_{\boldsymbol{n}}$ represent respectively a pure longitudinal wave mode $\boldsymbol{\gamma}_{\boldsymbol{n}, L}=\boldsymbol{n}$ and two pure transverse wave modes $\left\{\gamma_{\boldsymbol{n}, T_{\alpha}}=\boldsymbol{t}_{\alpha}\right\}_{\alpha=1,2}$.

\section{Unified variational framework for space $d G$ methods}

The basic idea of space dG methods is twofold. Firstly, we search for an approximated solution $\boldsymbol{U}_{h}(\boldsymbol{x}, t)=\left(\boldsymbol{v}_{h}(\boldsymbol{x}, t) \boldsymbol{\sigma}_{h}(\boldsymbol{x}, t)\right)^{T}$ of the generalized unknown $\boldsymbol{U}(\boldsymbol{x}, t)=(\boldsymbol{v}(\boldsymbol{x}, t) \boldsymbol{\sigma}(\boldsymbol{x}, t))^{T}$. In contrast to continuous finite element methods, this approximated solution is discontinuous from one element to another; hence the variational formulation can be developed independently in each element. As the fluxes coming from two adjacent elements are discontinuous on their inter-

face, the second idea is to replace them by a unique numerical flux, which in some way "glues" adjacent elements together.

Let's consider a finite element mesh $\mathscr{M}_{h}=\left\{\Omega_{k}\right\}_{k}$ of the domain $\Omega$. In the following, any element $\Omega_{k}$ of the mesh $\mathscr{M}_{h}$ will be denoted by $E$ and any of its neighboring elements by $E^{\prime}$. The discontinuous solutions in $E$ and $E^{\prime}$ are respectively denoted by $\boldsymbol{U}_{h}$ and $\boldsymbol{U}_{h}^{\prime}$.

The variational formulation of the elastic wave model problem (1 or 6) for any element $E$ is obtained as follows: $\forall \boldsymbol{W}_{h}(\boldsymbol{x})=\left(\boldsymbol{w}_{h}(\boldsymbol{x}) \boldsymbol{\tau}_{h}(\boldsymbol{x})\right)^{T}$

$$
\begin{aligned}
& \left(\boldsymbol{w}_{h}, \partial_{t} \boldsymbol{v}_{h}\right)_{E}-\left(\boldsymbol{w}_{h}, \rho^{-1} \boldsymbol{D i v}_{x} \boldsymbol{\sigma}_{h}\right)_{E}=0 \\
& \left(\boldsymbol{\tau}_{h}, \partial_{t} \boldsymbol{\sigma}_{h}\right)_{E}-\left(\boldsymbol{\tau}_{h}, \boldsymbol{C}: \boldsymbol{\varepsilon}\left(\boldsymbol{v}_{h}\right)\right)_{E}=0
\end{aligned}
$$

or,

$$
\left(\boldsymbol{W}_{h}, \partial_{t} \boldsymbol{U}_{h}\right)_{E}+\left(\boldsymbol{W}_{h}, \boldsymbol{A}^{\partial_{x}}\left(\boldsymbol{U}_{h}\right)\right)_{E}=0
$$

where the inner products are defined as follows by recalling the definition of the 230 dot product given in (7):

$$
\begin{aligned}
& \left(\boldsymbol{w}_{1}, \boldsymbol{w}_{2}\right)_{E}=\int_{E} \boldsymbol{w}_{1} \cdot \boldsymbol{w}_{2} \mathrm{~d} V,\left(\boldsymbol{\tau}_{1}, \boldsymbol{\tau}_{2}\right)_{E}=\int_{E} \boldsymbol{\tau}_{1}: \boldsymbol{\tau}_{2} \mathrm{~d} V \\
& \left(\boldsymbol{W}_{1}, \boldsymbol{W}_{2}\right)_{E}=\int_{E} \boldsymbol{W}_{1} \cdot \boldsymbol{W}_{2} \mathrm{~d} V=\left(\boldsymbol{w}_{1}, \boldsymbol{w}_{2}\right)_{E}+\left(\boldsymbol{\tau}_{1}, \boldsymbol{\tau}_{2}\right)_{E}
\end{aligned}
$$

Then, integration by parts of 24 or 25 yields:

$$
\begin{aligned}
& \left.\left(\boldsymbol{w}_{h}, \partial_{t} \boldsymbol{v}_{h}\right)_{E}+\left(\rho^{-1} \boldsymbol{\varepsilon}\left(\boldsymbol{w}_{h}\right), \boldsymbol{\sigma}_{h}\right)_{E}-<\boldsymbol{w}_{h}, \rho^{-1} \boldsymbol{\sigma}_{h} \cdot \boldsymbol{n}\right)>_{\partial E}=0 \\
& \left(\boldsymbol{\tau}_{h}, \partial_{t} \boldsymbol{\sigma}_{h}\right)_{E}+\left(\operatorname{Div}_{x}\left(\boldsymbol{C}: \boldsymbol{\tau}_{h}\right), \boldsymbol{v}_{h}\right)_{E}-<\boldsymbol{\tau}_{h}, \boldsymbol{C}:\left(\boldsymbol{n} \otimes_{s} \boldsymbol{v}_{h}\right)>_{\partial E}=0
\end{aligned}
$$

or,

$$
\left(\boldsymbol{W}_{h}, \partial_{t} \boldsymbol{U}_{h}\right)_{E}-\left(\boldsymbol{A}^{\partial_{x}, T}\left(\boldsymbol{W}_{h}\right), \boldsymbol{U}_{h}\right)_{E}+<\boldsymbol{W}_{h}, \boldsymbol{F}_{\boldsymbol{n}}\left(\boldsymbol{U}_{h}\right)>_{\partial E}=0
$$


where $<\cdot>_{\partial E}$ denotes the surface integration on the element boundary $\partial E$, and $\boldsymbol{A}^{\partial_{x}, T}$ is the transpose of $\boldsymbol{A}^{\partial_{x}}$ defined as: $\forall \boldsymbol{W}=(\boldsymbol{w} \boldsymbol{\tau})^{T}$

$$
\boldsymbol{A}^{\partial_{x}, T}\left(\begin{array}{c}
\boldsymbol{w} \\
\boldsymbol{\tau}
\end{array}\right)=\left(\begin{array}{c}
-\operatorname{Div}_{x}(\boldsymbol{C}: \tau) \\
-\rho^{-1} \varepsilon(\boldsymbol{w})
\end{array}\right)
$$

We notice that the flux operator $\boldsymbol{F}_{\boldsymbol{n}}$ already defined in $(10)$ is brought out in (27, 28).

As an interesting result, the well-known rotational invariance property of the isotropic elastic wave problem (6) or 9) can be easily proved within the presented tensorial variational framework. Indeed, by comparing the two definitions of $\boldsymbol{A}_{\boldsymbol{e}}$ (8) and of $\boldsymbol{A}_{\boldsymbol{n}}$ (10), we notice that, in the isotropic case, their matrix forms $\left[A_{\boldsymbol{e}_{1}}\right]$ expressed in the global basis $\left(\boldsymbol{e}_{1}, \boldsymbol{e}_{2}, \boldsymbol{e}_{3}\right)$ and $\left[A_{\boldsymbol{n}}\right]$ expressed in the local basis $\left(\boldsymbol{n}, \boldsymbol{t}_{1}, \boldsymbol{t}_{2}\right)$ - are identical (see Appendix A1).

\subsection{Upwind numerical flux on interior element interfaces}

Now, to complete the development of the $\mathrm{dG}$ variational formulation, it is necessary to replace the discontinuous flux $\boldsymbol{F}_{\boldsymbol{n}}\left(\boldsymbol{U}_{h}\right)$ on the interior element boundary $\partial E_{i n t}=\partial E \backslash(\partial E \cap \partial \Omega)$ by a numerical flux $\hat{\boldsymbol{F}}_{\boldsymbol{n}}\left(\boldsymbol{U}_{h}, \boldsymbol{U}_{h}^{\prime}\right)$, which should also depend on the solution $\boldsymbol{U}_{h}^{\prime}$ in the neighboring elements $E^{\prime}$ of $E$. As for the exterior element boundary $\partial E_{\text {ext }}=\partial E \cap \partial \Omega$, the definition of the numerical flux $\hat{\boldsymbol{F}}_{\boldsymbol{n}}\left(\boldsymbol{U}_{h}\right)$ should take into account the boundary conditions and will be considered in the next subsection.

In the elliptic case, the natural choice of numerical flux is the mean flux $\frac{1}{2}\left(\boldsymbol{F}_{\boldsymbol{n}}\left(\boldsymbol{U}_{h}\right)-\boldsymbol{F}_{\boldsymbol{n}^{\prime}}^{\prime}\left(\boldsymbol{U}^{\prime}{ }_{h}\right)\right)$, as there is no privileged characteristic direction inherent to the system of PDEs. We note that the two outward unit normal vectors of $E$ and $E^{\prime}$ on their interface verify $\boldsymbol{n}+\boldsymbol{n}^{\prime}=\mathbf{0}$.

As the elastic wave problem (6) is first-order hyperbolic, the principal idea of defining an appropriate numerical flux is the well-known Godunov flux by solving the Riemann problem defined on the element interfaces and leads to the use of Upwind numerical fluxes [4, 9, 12. In the present work, three upwind numerical fluxes are proposed and studied within the particular context of piecewise homogeneous media.

\subsubsection{Uncoupled upwind numerical flux}

The first numerical flux considered is derived from the one proposed by Käser et al. 2, which is expressed only in terms of material properties from the interior of the element $E$ from one side of element interface, i.e.,

$$
\hat{\boldsymbol{F}}_{\boldsymbol{n}}\left(\boldsymbol{U}_{h}, \boldsymbol{U}_{h}^{\prime}\right)=\left(\lambda_{\boldsymbol{n}, k}^{+} \boldsymbol{R}_{\boldsymbol{n}, k}^{+} \otimes \boldsymbol{L}_{\boldsymbol{n}, k}^{+}\right) \cdot \boldsymbol{U}_{h}+\left(\lambda_{\boldsymbol{n}, k}^{-} \boldsymbol{R}_{\boldsymbol{n}, k}^{-} \otimes \boldsymbol{L}_{\boldsymbol{n}, k}^{-}\right) \cdot \boldsymbol{U}_{h}^{\prime}
$$

265 By upwinding, the velocity and stress states $\boldsymbol{U}_{h}$ on the side of the element $E$ are only combined with the wave eigenmodes corresponding to the strictly positive eigenvalues of the flux operator, while the velocity and stress states $\boldsymbol{U}_{h}^{\prime}$ on the side of the neighboring element $E^{\prime}$ are combined with the wave eigenmodes corresponding to the strictly negative eigenvalues. On a physical interface, it is 

interface [13, as $\hat{\boldsymbol{F}}_{\boldsymbol{n}^{\prime}}\left(\boldsymbol{U}_{h}^{\prime}, \boldsymbol{U}_{h}\right) \neq-\hat{\boldsymbol{F}}_{\boldsymbol{n}}\left(\boldsymbol{U}_{h}, \boldsymbol{U}_{h}^{\prime}\right)$.

Herein, this numerical flux is modified in order to use independently material properties coming from elements on both sides of physical interfaces:

$$
\hat{\boldsymbol{F}}_{\boldsymbol{n}}\left(\boldsymbol{U}_{h}, \boldsymbol{U}_{h}^{\prime}\right)=\left(\lambda_{\boldsymbol{n}, k}^{+} \boldsymbol{R}_{\boldsymbol{n}, k}^{+} \otimes \boldsymbol{L}_{\boldsymbol{n}, k}^{+}\right) \cdot \boldsymbol{U}_{h}-\left(\lambda_{\boldsymbol{n}^{\prime}, k}^{\prime+} \boldsymbol{R}_{\boldsymbol{n}^{\prime}, k}^{+} \otimes \boldsymbol{L}_{\boldsymbol{n}^{\prime}, k}^{\prime+}\right) \cdot \boldsymbol{U}_{h}^{\prime}
$$

The above numerical flux is called "uncoupled" upwind flux in the present work 275 as it does not imply further coupling between $(\boldsymbol{C}, \rho, \boldsymbol{U})$ and $\left(\boldsymbol{C}^{\prime}, \rho^{\prime}, \boldsymbol{U}^{\prime}\right)$ of two adjacent elements $E$ and $E^{\prime}$.

It is worth indicating that, when there are no discontinuities of material properties between $E$ and $E^{\prime},(31)$ is identical to (30), because we have in this case $\lambda_{\boldsymbol{n}, k}^{-}=-\lambda_{\boldsymbol{n}^{\prime}, k}^{\prime+}, \boldsymbol{R}_{\boldsymbol{n}, k}^{-}=\boldsymbol{R}_{\boldsymbol{n}^{\prime}, k}^{\prime+1}$ and $\boldsymbol{L}_{\boldsymbol{n}, k}^{-}=\boldsymbol{L}_{\boldsymbol{n}^{\prime}, k}^{\prime+}$.

By replacing the flux terms in (28) by the numerical flux (31), we get the following $\mathrm{dG}$ variational formulation:

$$
\begin{aligned}
& 0=\left(\boldsymbol{W}_{h}, \partial_{t} \boldsymbol{U}_{h}\right)_{E}-\left(\boldsymbol{A}^{\partial_{x}, T}\left(\boldsymbol{W}_{h}\right), \boldsymbol{U}_{h}\right)_{E}+<\boldsymbol{W}_{h}, \hat{\boldsymbol{F}}_{h}\left(\boldsymbol{U}_{h}\right)>_{\partial E_{e x t}} \\
& +<\lambda_{\boldsymbol{n}, k}^{+} \boldsymbol{R}_{\boldsymbol{n}, k}^{+} \cdot \boldsymbol{W}_{h}, \boldsymbol{L}_{\boldsymbol{n}, k}^{+} \cdot \boldsymbol{U}_{h}>_{\partial E_{i n t}}-<\lambda_{\boldsymbol{n}^{\prime}, k}^{\prime+} \boldsymbol{R}_{\boldsymbol{n}^{\prime}, k}^{\prime+} \cdot \boldsymbol{W}_{h}, \boldsymbol{L}_{\boldsymbol{n}^{\prime}, k}^{\prime+} \cdot \boldsymbol{U}_{h}^{\prime}>_{\partial E_{i n t}}
\end{aligned}
$$

We can also obtain another dG variational formulation equivalent to $\sqrt{32}$ by applying once more the integration by parts to the latter and by using 23):

$$
\begin{aligned}
& 0=\left(\boldsymbol{W}_{h}, \partial_{t} \boldsymbol{U}_{h}\right)_{E}+\left(\boldsymbol{W}_{h}, \boldsymbol{A}^{\partial_{x}}\left(\boldsymbol{U}_{h}\right)\right)_{E}+<\boldsymbol{W}_{h}, \hat{\boldsymbol{F}}_{n}\left(\boldsymbol{U}_{h}\right)-\boldsymbol{F}_{n}\left(\boldsymbol{U}_{h}\right)>_{\partial E_{e x t}} \\
& -<\lambda_{\boldsymbol{n}, k}^{-} \boldsymbol{R}_{\boldsymbol{n}, k}^{-} \cdot \boldsymbol{W}_{h}, \boldsymbol{L}_{\boldsymbol{n}, k}^{-} \cdot \boldsymbol{U}_{h}>_{\partial E_{i n t}}-<\lambda_{\boldsymbol{n}^{\prime}, k}^{\prime+} \boldsymbol{R}_{\boldsymbol{n}^{\prime}, k}^{\prime+} \cdot \boldsymbol{W}_{h}, \boldsymbol{L}_{\boldsymbol{n}^{\prime}, k}^{\prime+} \cdot \boldsymbol{U}_{h}^{\prime}>_{\partial E_{i n t}}
\end{aligned}
$$

This second variational dG formulation is also called "strong form" by some authors, as it explicitly contains the differential operator $\boldsymbol{A}^{\partial_{x}}$ involved in the strong formulation of the elastic wave problem (6), contrary to the formulation (32) invoking the operator $\boldsymbol{A}^{\partial_{x}, T}$. Both formulations (32) and (33) are equivalent and we have chosen to implement the latter one, i.e. the "strong form", because the terms involved in the differential operator $\boldsymbol{A}^{\partial_{x}}$ are slightly more

\subsubsection{Coupled upwind numerical fluxes}

Two other numerical fluxes considered in the present work are defined by writing the formulation (33) using averaged materials properties on element interfaces:

$$
\begin{aligned}
& 0=\left(\boldsymbol{W}_{h}, \partial_{t} \boldsymbol{U}_{h}\right)_{E}+\left(\boldsymbol{W}_{h}, \boldsymbol{A}^{\partial_{x}}\left(\boldsymbol{U}_{h}\right)\right)_{E}+<\boldsymbol{W}_{h}, \hat{\boldsymbol{F}}_{n}\left(\boldsymbol{U}_{h}\right)-\boldsymbol{F}_{n}\left(\boldsymbol{U}_{h}\right)>_{\partial E_{e x t}} \\
& -<\bar{\lambda}_{\boldsymbol{n}, k}^{-} \overline{\boldsymbol{R}}_{\boldsymbol{n}, k}^{-} \cdot \boldsymbol{W}_{h}, \overline{\boldsymbol{L}}_{\boldsymbol{n}, k}^{-} \cdot \boldsymbol{U}_{h}>_{\partial E_{i n t}}-<\bar{\lambda}_{\boldsymbol{n}^{\prime}, k}^{+} \overline{\boldsymbol{R}}_{\boldsymbol{n}^{\prime}, k}^{+} \cdot \boldsymbol{W}_{h}, \overline{\boldsymbol{L}}_{\boldsymbol{n}^{\prime}, k}^{+} \cdot \boldsymbol{U}_{h}^{\prime}>_{\partial E_{i n t}}
\end{aligned}
$$

where $\left(\bar{\lambda}_{\boldsymbol{n}, k}^{-}, \overline{\boldsymbol{R}}_{\boldsymbol{n}, k}^{-}, \overline{\boldsymbol{L}}_{\boldsymbol{n}, k}^{-}\right)$are the eigenvalues and eigenvectors defined by the averaged material properties $(\overline{\boldsymbol{C}}, \bar{\rho})$ on element interfaces. Two methods to calculate averaged material properties are considered in this work:

(1) The first method calculates the arithmetic average for the Hooke tensors and the harmonic average for the densities: $\bar{C}=\boldsymbol{C}^{\text {Voigt }}=\left(\boldsymbol{C}+\boldsymbol{C}^{\prime}\right) / 2$ 
and $\left(\bar{\rho}^{\text {Reuss }}\right)^{-1}=\left(\rho^{-1}+\rho^{-1}\right) / 2$. The so-obtained averaged elastic tensor $C^{\text {Voigt }}$ is usually called the Voigt average. The corresponding numerical flux is named "cV_Flux" hereafter.

(2) The second method calculates the harmonic average for the Hooke tensors and the arithmetic average for the densities: $\overline{\boldsymbol{C}}^{-1}=\left(\boldsymbol{C}^{\text {Reuss }}\right)^{-1}=\left(\boldsymbol{C}^{-1}+\right.$ $\left.\boldsymbol{C}^{\prime-1}\right) / 2$ and $\bar{\rho}^{\text {Voigt }}=\left(\rho+\rho^{\prime}\right) / 2$. The so-obtained averaged elastic tensor $C^{\text {Reuss }}$ is usually called Reuss average. The corresponding numerical flux is named "cR_Flux" hereafter.

Both fluxes are called "coupled" upwind fluxes by contrast with the previously proposed "uncoupled" numerical flux.

When the material properties are continuous on element interfaces, it can be shown that the three numerical fluxes and the one proposed by Käser et al. are identical and are the exact solution of the Riemann problem in both isotropic and anisotropic cases. But this is no longer true in more general cases where adjacent elements have different material properties. In such cases, they only solve approximately the Riemann problem at those element interfaces and lead to different $\mathrm{dG}$ formulations. This is why it should be necessary to check if they keep working well. To do this, they are numerically investigated in the next section in several situations: 1D and 2D cases with only one interior physical interface and 2D polycrystalline materials in which a large number of physical interfaces exist as elastic properties are discontinuous from one crystallite to another. Otherwise, to get further investigations, two upwind numerical fluxes that are exact solutions of the Riemann problem are developed in the 1D case and are compared to the two coupled numerical fluxes.

\subsubsection{Upwind fluxes that are exact solutions of the Riemann problem in the $1 D$ case}

Two upwind fluxes that are exact solutions of the Riemann problem at element interfaces are introduced and considered in the 1D case. They are obtained from two equivalent strong forms of elastic wave equations. As one of the main results of the present work, we will show that solving exactly the Riemann problem at element interfaces is not a sufficient condition to get physically sound numerical solutions. Indeed, the local (that is, pointwise) wave equation can be written in different equivalent strong forms. However, when these strong forms are put into a weak form and integrated by part to get flux terms, they give rise to different interface conditions in terms of velocity and stress fields, which are 335 not all physically coherent.

For the first upwind flux, the Riemann problem is defined by directly considering the first-order velocity-stress system (6), while for the second one, it is modified by taking into account the classical mechanical interface conditions in terms of velocity and stress vector fields. The first flux is named "G_Flux" with "G" standing for "Godunov", while the second flux is named "MG_Flux" with "MG" standing for "mechanically based Godunov". 
I. G_Flux: Upwind flux directly derived from the first-order $\boldsymbol{v}-\boldsymbol{\sigma}$ system (6)

When the interface of two adjacent elements $E$ and $E^{\prime}$ having respectively $\left(\rho, \boldsymbol{C}, \boldsymbol{U}_{h}\right)$ and $\left(\rho^{\prime}, \boldsymbol{C}^{\prime}, \boldsymbol{U}_{h}^{\prime}\right)$ as densities, elastic moduli and initial states is considered (Figure 1), the Riemann problem defines the states that are results of the propagation of the discontinuity $\boldsymbol{U}_{h}-\boldsymbol{U}^{\prime}{ }_{h}$. As in the $1 \mathrm{D}$ case, there are only one positive eigenvalue and only one negative eigenvalue, the subscript " $k$ " in $\left(\lambda_{\boldsymbol{n}, k}^{ \pm}, \boldsymbol{R}_{\boldsymbol{n}, k}^{ \pm}, \boldsymbol{L}_{\boldsymbol{n}, k}^{ \pm}\right)$is omitted. Then the following Rankine-Hugoniot jump conditions hold for the first-order velocity-stress system (6) [7, 9, 12]:

$$
\begin{aligned}
\boldsymbol{A}_{\boldsymbol{n}}\left(\boldsymbol{U}_{h}-\boldsymbol{U}_{h}^{a}\right) & =\lambda_{\boldsymbol{n}}^{-}\left(\boldsymbol{U}_{h}-\boldsymbol{U}_{h}^{a}\right) \\
\boldsymbol{A}_{\boldsymbol{n}}\left(\boldsymbol{U}_{h}^{a}\right)+\boldsymbol{A}_{\boldsymbol{n}^{\prime}}^{\prime}\left(\boldsymbol{U}_{h}^{a^{\prime}}\right) & =\mathbf{0} \\
\boldsymbol{A}_{\boldsymbol{n}^{\prime}}^{\prime}\left(\boldsymbol{U}_{h}^{\prime}-\boldsymbol{U}_{h}^{a^{\prime}}\right) & =\lambda_{\boldsymbol{n}^{\prime}}^{-\prime}\left(\boldsymbol{U}_{h}^{\prime}-\boldsymbol{U}_{h}^{a^{\prime}}\right)
\end{aligned}
$$

According to the definition of the eigen problem of $\left.\boldsymbol{A}_{\boldsymbol{n}} \sqrt{13}\right)$, we can write:

$$
\boldsymbol{U}_{h}-\boldsymbol{U}_{h}^{a}=\alpha \boldsymbol{R}_{\boldsymbol{n}}^{-}, \boldsymbol{U}_{h}^{\prime}-\boldsymbol{U}_{h}^{a^{\prime}}=\alpha^{\prime} \boldsymbol{R}_{\boldsymbol{n}^{\prime}}^{{ }^{\prime}}
$$

the Rankine-Hugoniot jump conditions (35) becomes:

$$
\begin{aligned}
\boldsymbol{A}_{\boldsymbol{n}}\left(\boldsymbol{U}_{h}\right)-\boldsymbol{A}_{\boldsymbol{n}}\left(\boldsymbol{U}_{h}^{a}\right) & =\alpha \lambda_{\boldsymbol{n}}^{-} \boldsymbol{R}_{\boldsymbol{n}}^{-} \\
\boldsymbol{A}_{\boldsymbol{n}}\left(\boldsymbol{U}_{h}^{a}\right)+\boldsymbol{A}_{\boldsymbol{n}^{\prime}}^{\prime}\left(\boldsymbol{U}_{h}^{a^{\prime}}\right) & =\mathbf{0} \\
\boldsymbol{A}_{\boldsymbol{n}^{\prime}}^{\prime}\left(\boldsymbol{U}_{h}^{\prime}\right)-\boldsymbol{A}_{\boldsymbol{n}^{\prime}}^{\prime}\left(\boldsymbol{U}_{h}^{a^{\prime}}\right) & =\alpha^{\prime} \lambda_{\boldsymbol{n}^{\prime}}^{-\boldsymbol{R}_{\boldsymbol{n}^{\prime}}^{\prime}}
\end{aligned}
$$

Solving the Riemann problem (37) leads to the determination of the two unknown states $\left(\boldsymbol{U}^{a}, \boldsymbol{U}^{a^{\prime}}\right)$, i.e. the two characteristic coefficients $\left(\alpha, \alpha^{\prime}\right)$. Then the upwind numerical fluxes defined as $\hat{\boldsymbol{F}}_{\boldsymbol{n}}\left(\boldsymbol{U}_{h}, \boldsymbol{U}_{h}^{\prime}\right)=\boldsymbol{A}_{\boldsymbol{n}}\left(\boldsymbol{U}_{h}^{a}\right)=\frac{1}{2}\left(\boldsymbol{A}_{\boldsymbol{n}}\left(\boldsymbol{U}^{a}\right)-\right.$ $\left.\boldsymbol{A}_{\boldsymbol{n}^{\prime}}^{\prime}\left(\boldsymbol{U}_{h}^{a^{\prime}}\right)\right)$ can be calculated using the following two equivalent forms:

$$
\begin{aligned}
\hat{\boldsymbol{F}}_{\boldsymbol{n}}\left(\boldsymbol{U}_{h}, \boldsymbol{U}_{h}^{\prime}\right) & =\frac{1}{2}\left(\boldsymbol{A}_{\boldsymbol{n}}\left(\boldsymbol{U}_{h}\right)-\boldsymbol{A}_{\boldsymbol{n}^{\prime}}^{\prime}\left(\boldsymbol{U}_{h}^{\prime}\right)-\alpha \lambda_{\boldsymbol{n}}^{-} \boldsymbol{R}_{\boldsymbol{n}}^{-}+\alpha^{\prime} \lambda_{\boldsymbol{n}}^{-^{\prime}} \boldsymbol{R}_{\boldsymbol{n}}^{-^{\prime}}\right) \\
& =\boldsymbol{A}_{\boldsymbol{n}}\left(\boldsymbol{U}_{h}\right)-\alpha \lambda_{\boldsymbol{n}}^{-} \boldsymbol{R}_{\boldsymbol{n}}^{-}
\end{aligned}
$$

Considering (35), it is obvious that the flux is conservative, i.e. from the point 345 of view of the neighboring element $E^{\prime}$, we have $\hat{\boldsymbol{F}}_{n^{\prime}}\left(\boldsymbol{U}_{h}^{\prime}, \boldsymbol{U}_{h}\right)=-\hat{\boldsymbol{F}}_{\boldsymbol{n}}\left(\boldsymbol{U}_{h}, \boldsymbol{U}_{h}^{\prime}\right)$.

In the $1 \mathrm{D}$ case, $\left(\alpha, \alpha^{\prime}\right)$ can be easily obtained (see Appendix A2):

$$
\alpha={ }^{*} \boldsymbol{L}_{\boldsymbol{n}}^{-} \cdot \boldsymbol{U}_{h}-{ }^{* *} \boldsymbol{L}_{\boldsymbol{n}}^{-} \cdot \boldsymbol{U}_{h}^{\prime}, \alpha^{\prime}={ }^{*} \boldsymbol{L}_{\boldsymbol{n}^{\prime}}^{-^{\prime}} \cdot \boldsymbol{U}_{h}^{\prime}-{ }^{* *} \boldsymbol{L}_{\boldsymbol{n}^{\prime}}^{{ }^{\prime}} \cdot \boldsymbol{U}_{h}
$$

In (38), $\left({ }^{*} \boldsymbol{L}_{\boldsymbol{n}}^{-},{ }^{* *} \boldsymbol{L}_{\boldsymbol{n}}^{-}\right)$and $\left({ }^{*} \boldsymbol{L}_{\boldsymbol{n}^{\prime}}^{-},{ }^{* *} \boldsymbol{L}_{\boldsymbol{n}^{\prime}}^{-\prime}\right)$ are respectively the modified left eigenmodes of $\boldsymbol{A}_{\boldsymbol{n}}$ and $\boldsymbol{A}_{\boldsymbol{n}^{\prime}}^{\prime}$, which are calculated in the following way by using the 
material properties of the adjacent element:

$$
\begin{gathered}
{ }^{*} \boldsymbol{L}_{\boldsymbol{n}}^{-}=\left(\begin{array}{c}
C_{z}^{\prime} \wp_{\text {vect }}\left(\boldsymbol{L}_{\boldsymbol{n}}^{-}\right) \\
C_{z} \wp_{\text {tens }}\left(\boldsymbol{L}_{\boldsymbol{n}}^{-}\right)
\end{array}\right),{ }^{* *} \boldsymbol{L}_{\boldsymbol{n}}^{-}=\left(\begin{array}{c}
\frac{\rho^{\prime}}{\rho} \frac{\boldsymbol{\Gamma}_{\boldsymbol{n}^{\prime}}^{\prime} \cdot \wp_{\text {vect }}\left({ }^{*} \boldsymbol{L}_{\boldsymbol{n}}^{-}\right)}{\lambda_{\boldsymbol{n}}^{-2}} \\
\frac{\rho}{\rho^{\prime}} \wp_{\text {tens }}\left({ }^{*} \boldsymbol{L}_{\boldsymbol{n}}^{-}\right)
\end{array}\right) \\
{ }^{*} \boldsymbol{L}_{\boldsymbol{n}^{\prime}}^{-^{\prime}}=\left(\begin{array}{c}
C_{z} \wp_{\text {vect }}\left(\boldsymbol{L}_{\boldsymbol{n}^{\prime}}^{-^{\prime}}\right) \\
C_{z}^{\prime} \wp_{\text {tens }}\left(\boldsymbol{L}_{\boldsymbol{n}^{\prime}}^{-^{\prime}}\right)
\end{array}\right),{ }^{* *} \boldsymbol{L}_{\boldsymbol{n}^{\prime}}^{-^{\prime}}=\left(\begin{array}{c}
\frac{\rho}{\rho^{\prime}} \frac{\left.\boldsymbol{\Gamma}_{\boldsymbol{n}} \cdot \wp_{\text {vect }}{ }^{*} \boldsymbol{L}_{\boldsymbol{n}^{\prime}}^{-^{\prime}}\right)}{\lambda_{\boldsymbol{n}^{\prime}}^{\prime^{\prime}}} \\
\frac{\rho^{\prime}}{\rho} \wp_{\text {tens }}\left({ }^{*} \boldsymbol{L}_{\boldsymbol{n}^{\prime}}^{-^{\prime}}\right)
\end{array}\right)
\end{gathered}
$$

with:

$$
C_{z}=\frac{{\overline{\rho \lambda_{n}^{-}}}^{\text {Reuss }}}{\rho \lambda_{n}^{-}}=\frac{\rho^{\prime} \lambda_{\boldsymbol{n}}^{-^{\prime}}}{\overline{\rho \lambda_{n}^{-}}}, C_{z}^{\prime}=\frac{{\overline{\rho \lambda_{n}^{-}}}^{\text {Reuss }}}{\rho^{\prime} \lambda_{\boldsymbol{n}^{\prime}}^{-\prime}}=\frac{\rho \lambda_{\boldsymbol{n}}^{-}}{{\overline{\rho \lambda_{\boldsymbol{n}}^{-}}}^{\text {Voigt }}}
$$

where ${\overline{\rho \lambda_{n}^{-}}}^{\text {Reuss }}$ and ${\overline{\rho \lambda_{n}^{-}}}^{\text {Voigt }}$ denote respectively the harmonic and arithmetic averages of acoustic impedances $\rho \lambda_{\boldsymbol{n}, k}^{-}$and $\rho^{\prime} \lambda_{\boldsymbol{n}^{\prime}, k}^{-^{\prime}}$, and the two operators $\wp_{\text {vect }}$ and $\wp_{\text {tens }}$ are defined as follows: $\forall \boldsymbol{W}=(\boldsymbol{w} \boldsymbol{\tau})^{T}$,

$$
\wp_{\text {vect }}(\boldsymbol{W})=\boldsymbol{w}, \wp_{\text {tens }}(\boldsymbol{W})=\boldsymbol{\tau}
$$

It is interesting to point out that the coupling terms highlighted in 40 are complex and do not correspond neither to the method with arithmetic average of $\boldsymbol{C} /$ harmonic average of $\rho$ nor to the one with harmonic average of $\boldsymbol{C} /$ arithmetic average of $\rho$. One important parameter to take into account is the acoustic impedance of the eigenmodes.

Finally, the most important remark concerning the numerical flux (38) is that, the equation (35b) of the Rankine-Hugoniot jump conditions (35), which is directly derived from the first-order velocity-stress system (6), leads to the following interface conditions:

$$
\frac{\boldsymbol{\sigma}_{h}^{a} \cdot \boldsymbol{n}}{\rho}+\frac{\boldsymbol{\sigma}_{h}^{a^{\prime}} \cdot \boldsymbol{n}^{\prime}}{\rho^{\prime}}=\mathbf{0}, \rho \boldsymbol{\Gamma}_{\boldsymbol{n}} \cdot \boldsymbol{v}_{h}^{a}-\rho^{\prime} \boldsymbol{\Gamma}_{\boldsymbol{n}^{\prime}}^{\prime} \cdot \boldsymbol{v}_{h}^{a^{\prime}}=\mathbf{0}
$$

It is obvious that, on a physical interface, i.e. $\rho \neq \rho^{\prime}$ or/and $\boldsymbol{\Gamma}_{\boldsymbol{n}} \neq \boldsymbol{\Gamma}_{\boldsymbol{n}^{\prime}}^{\prime}$, the conditions 43 are not equivalent to the classical interface conditions of continuous velocity and stress vector fields, the resulting flux $(38)$ is therefore not physically sound. Indeed, in Section 4.1 it will be shown that this numerical flux cannot give rise to physically sound wave propagation solutions.

II. MG_Flux: Upwind flux based on the mechanical interface conditions

In order to remove the physical inconsistency of the interface conditions 43, 
we propose to modify them so that the following classical interface conditions are recovered:

$$
\boldsymbol{\sigma}_{h}^{a} \cdot \boldsymbol{n}+\boldsymbol{\sigma}_{h}^{a^{\prime}} \cdot \boldsymbol{n}^{\prime}=\mathbf{0}, \boldsymbol{n} \otimes_{s} \boldsymbol{v}_{h}^{a}+\boldsymbol{n}^{\prime} \otimes_{s} \boldsymbol{v}_{h}^{a^{\prime}}=\mathbf{0}
$$

It is easy to show that the interface conditions (43) and (44) differ only when the elastic moduli or/and the density of the propagating medium are discontinuous.

The interface conditions (44) can be in fact considered as consequence of the following Rankine-Hugoniot jump conditions:

$$
\begin{aligned}
\tilde{\boldsymbol{A}}_{\boldsymbol{n}}\left(\boldsymbol{U}_{h}\right)-\tilde{\boldsymbol{A}}_{\boldsymbol{n}}\left(\boldsymbol{U}_{h}^{a}\right) & =\tilde{\alpha} \lambda_{\boldsymbol{n}}^{-} \boldsymbol{M}\left(\boldsymbol{R}_{\boldsymbol{n}}^{-}\right) \\
\tilde{\boldsymbol{A}}_{\boldsymbol{n}}\left(\boldsymbol{U}_{h}^{a}\right)+\tilde{\boldsymbol{A}}_{\boldsymbol{n}^{\prime}}^{\prime}\left(\boldsymbol{U}_{h}^{a^{\prime}}\right) & =\mathbf{0} \\
\tilde{\boldsymbol{A}}_{\boldsymbol{n}^{\prime}}^{\prime}\left(\boldsymbol{U}_{h}^{\prime}\right)-\tilde{\boldsymbol{A}}_{\boldsymbol{n}^{\prime}}^{\prime}\left(\boldsymbol{U}_{h}^{a^{\prime}}\right) & =\tilde{\alpha}^{\prime} \lambda_{\boldsymbol{n}^{\prime}}^{-^{\prime}} \boldsymbol{M}^{\prime}\left(\boldsymbol{R}_{\boldsymbol{n}^{\prime}}^{-^{\prime}}\right)
\end{aligned}
$$

which correspond to another equivalent form of the studied first-order velocitystress system (6):

$$
\boldsymbol{M}\left(\partial_{t} \boldsymbol{U}\right)+\tilde{\boldsymbol{A}}^{\partial_{x}}(\boldsymbol{U})=\mathbf{0} \text { or } \begin{aligned}
\rho \partial_{t} \boldsymbol{v}-\mathbf{D i v}_{x} \boldsymbol{\sigma} & =\mathbf{0} \\
\boldsymbol{C}^{-1}: \partial_{t} \boldsymbol{\sigma}-\boldsymbol{\varepsilon}(\boldsymbol{v}) & =\mathbf{0}
\end{aligned}
$$

To complete the previously introduced equations, the following definitions of operators are necessary:

$$
\begin{gathered}
\boldsymbol{M}\left(\begin{array}{c}
\boldsymbol{w} \\
\boldsymbol{\tau}
\end{array}\right)=\left(\begin{array}{c}
\rho \boldsymbol{w} \\
\boldsymbol{C}^{-1}: \boldsymbol{\tau}
\end{array}\right), \boldsymbol{M}^{-1}\left(\begin{array}{c}
\boldsymbol{w} \\
\boldsymbol{\tau}
\end{array}\right)=\left(\begin{array}{c}
\rho^{-1} \boldsymbol{w} \\
\boldsymbol{C}: \boldsymbol{\tau}
\end{array}\right) \\
\tilde{\boldsymbol{A}}^{\partial_{x}}\left(\begin{array}{c}
\boldsymbol{w} \\
\boldsymbol{\tau}
\end{array}\right)=\left(\begin{array}{c}
-\operatorname{Div}_{x} \boldsymbol{\tau} \\
-\boldsymbol{\varepsilon}(\boldsymbol{w})
\end{array}\right), \tilde{\boldsymbol{A}}_{\boldsymbol{n}}\left(\begin{array}{c}
\boldsymbol{w} \\
\boldsymbol{\tau}
\end{array}\right)=\left(\begin{array}{c}
-\boldsymbol{\tau} \cdot \boldsymbol{n} \\
-\boldsymbol{n} \otimes_{s} \boldsymbol{w}
\end{array}\right)
\end{gathered}
$$

The following equations are straightforward:

$$
\boldsymbol{A}_{\boldsymbol{n}}=\boldsymbol{M}^{-1} \cdot \tilde{\boldsymbol{A}}_{\boldsymbol{n}}, \boldsymbol{M}\left(\boldsymbol{R}_{\boldsymbol{n}, k}^{ \pm}\right)=\rho \boldsymbol{L}_{\boldsymbol{n}, k}^{ \pm}
$$

As in the case of "G_Flux" and using (48), when the characteristic coefficients $\left(\tilde{\alpha}, \tilde{\alpha}^{\prime}\right)$ are calculated, the upwind numerical fluxes are defined as follows:

$$
\begin{aligned}
\hat{\boldsymbol{F}}_{\boldsymbol{n}}\left(\boldsymbol{U}_{h}, \boldsymbol{U}_{h}^{\prime}\right)=\boldsymbol{M}^{-1} \cdot \hat{\tilde{\boldsymbol{F}}}_{\boldsymbol{n}}\left(\boldsymbol{U}_{h}, \boldsymbol{U}_{h}^{\prime}\right) & =\boldsymbol{M}^{-1} \cdot\left(\tilde{\boldsymbol{A}}_{\boldsymbol{n}}\left(\boldsymbol{U}_{h}\right)-\tilde{\alpha} \lambda_{\boldsymbol{n}}^{-} \boldsymbol{M}\left(\boldsymbol{R}_{\boldsymbol{n}}^{-}\right)\right) \\
& =\boldsymbol{A}_{\boldsymbol{n}}\left(\boldsymbol{U}_{h}\right)-\tilde{\alpha} \lambda_{\boldsymbol{n}}^{-} \boldsymbol{R}_{\boldsymbol{n}}^{-}
\end{aligned}
$$

It is worth noticing that $\hat{\boldsymbol{F}}_{\boldsymbol{n}^{\prime}}\left(\boldsymbol{U}_{h}^{\prime}, \boldsymbol{U}_{h}\right) \neq-\hat{\boldsymbol{F}}_{\boldsymbol{n}}\left(\boldsymbol{U}_{h}, \boldsymbol{U}_{h}^{\prime}\right)$, even if $\hat{\tilde{\boldsymbol{F}}}_{\boldsymbol{n}^{\prime}}\left(\boldsymbol{U}_{h}^{\prime}, \boldsymbol{U}_{h}\right)=$ ${ }_{380}-\hat{\tilde{\boldsymbol{F}}}_{\boldsymbol{n}}\left(\boldsymbol{U}_{h}, \boldsymbol{U}_{h}^{\prime}\right)$.

As for the case of "G_Flux", $\left(\tilde{\alpha}, \tilde{\alpha}^{\prime}\right)$ can be easily obtained (see Appendix A3):

$$
\tilde{\alpha}={ }^{\sim} \boldsymbol{L}_{\boldsymbol{n}}^{-} \cdot\left(\boldsymbol{U}_{h}-\boldsymbol{U}_{h}^{\prime}\right), \tilde{\alpha}^{\prime}={ }^{\sim} \boldsymbol{L}_{\boldsymbol{n}^{\prime}}^{-^{\prime}} \cdot\left(\boldsymbol{U}_{h}^{\prime}-\boldsymbol{U}_{h}\right)
$$

where ${ }^{\sim} \boldsymbol{L}_{\boldsymbol{n}}^{-}$and ${ }^{\sim} \boldsymbol{L}_{\boldsymbol{n}^{\prime}}^{-{ }^{\prime}}$ are respectively the modified left eigenmodes of $\boldsymbol{A}_{\boldsymbol{n}}$ and $\boldsymbol{A}_{\boldsymbol{n}^{\prime}}^{\prime}$, which are calculated in the following way by using the material properties 
of the adjacent element:

$$
{ }^{\boldsymbol{L}_{\boldsymbol{n}}^{-}}=\left(\begin{array}{c}
C_{z} \wp_{\text {vect }}\left(\boldsymbol{L}_{\boldsymbol{n}}^{-}\right) \\
C_{z \wp_{\text {tens }}^{\prime}}^{\prime}\left(\boldsymbol{L}_{\boldsymbol{n}}^{-}\right)
\end{array}\right), \boldsymbol{L}_{\boldsymbol{n}^{\prime}}^{-^{\prime}}=\left(\begin{array}{c}
C_{z}^{\prime} \wp_{\text {vect }}\left(\boldsymbol{L}_{\boldsymbol{n}^{\prime}}^{-^{\prime}}\right) \\
C_{z \wp_{\text {tens }}}\left(\boldsymbol{L}_{\boldsymbol{n}^{\prime}}^{-^{\prime}}\right.
\end{array}\right)
$$

Two important remarks can be made. Firstly, as previously mentioned, two mathematically equivalent systems can lead to different flux definitions, it is important to check their physical meaning and their relevance. Secondly, the first-order velocity-strain wave equations used in [7] provides another way to get the Rankine-Hugoniot jump conditions that are coherent with the mechanical interface conditions.

\subsection{Boundary conditions}

As for the classical finite element method, the Neumann boundary conditions are weakly imposed through the variational framework. Indeed, by decomposing the numerical flux as $\hat{\boldsymbol{F}}_{\boldsymbol{n}}\left(\boldsymbol{U}_{h}\right)=\left(\hat{\boldsymbol{F}}_{\boldsymbol{n}, v}\left(\boldsymbol{U}_{h}\right) \hat{\boldsymbol{F}}_{\boldsymbol{n}, \sigma}\left(\boldsymbol{U}_{h}\right)\right)^{T}$ and according to the boundary conditions $\sqrt{11})$, it is natural to impose the part $\hat{\boldsymbol{F}}_{\boldsymbol{n}, v}\left(\boldsymbol{U}_{h}\right)$ equal to the Neumann boundary conditions. While the other part $\hat{\boldsymbol{F}}_{\boldsymbol{n}, \sigma}\left(\boldsymbol{U}_{h}\right)$ should be completely determined by the element $E$, as there is no neighboring element. We get:

$$
\hat{\boldsymbol{F}}_{n}\left(\boldsymbol{U}_{h}\right)=\left(\begin{array}{c}
-\rho^{-1} \boldsymbol{g}(\boldsymbol{x}, t) \\
\boldsymbol{F}_{\boldsymbol{n}, \sigma}\left(\boldsymbol{U}_{h}\right)
\end{array}\right)
$$

Concerning the Dirichlet boundary conditions, they are strongly imposed using a well-known strategy in the following way: $\boldsymbol{U}_{h}$ in $(33)$ is replaced by the sum of a solution with homogeneous Dirichlet boundary conditions $\boldsymbol{U}_{h}^{0}$ and of $\tilde{\boldsymbol{U}}_{D}=\left(\tilde{\boldsymbol{u}}_{D} \mathbf{0}\right)^{T}$, with $\tilde{\boldsymbol{u}}_{D}$ a lifting to $\Omega$ of $\boldsymbol{u}_{D}$ defined on $\partial \Omega_{D}$. The prescribed degrees of freedom of velocity are eliminated from $(33)$, which is solved with the terms containing $\tilde{\boldsymbol{U}}_{D}$ put on the right-hand side and treated as external loading terms.

\subsection{Element-wise $d G$ variational formulation}

By taking into account the Neumann and Dirichlet boundary conditions in (33), we get the following element-wise variational formulation of space dG method with, for example, the uncoupled upwind numerical flux:

$$
\begin{aligned}
& \left(\boldsymbol{W}_{h}, \partial_{t} \boldsymbol{U}_{h}\right)_{E}+\left(\boldsymbol{W}_{h}, \boldsymbol{A}^{\partial_{x}}\left(\boldsymbol{U}_{h}\right)\right)_{E} \\
-\quad & <\lambda_{\boldsymbol{n}, k}^{-\boldsymbol{R}_{\boldsymbol{n}, k}^{-}} \cdot \boldsymbol{W}_{h}, \boldsymbol{L}_{\boldsymbol{n}, k}^{-} \cdot \boldsymbol{U}_{h}>_{\partial E_{i n t}}-<\lambda_{\boldsymbol{n}^{\prime}, k}^{\prime+} \boldsymbol{R}_{\boldsymbol{n}^{\prime}, k}^{\prime+} \cdot \boldsymbol{W}_{h}, \boldsymbol{L}_{\boldsymbol{n}^{\prime}, k}^{\prime+} \cdot \boldsymbol{U}_{h}^{\prime}>_{\partial E_{i n t}} \\
+\quad & <\boldsymbol{w}_{h}, \rho^{-1} \boldsymbol{\sigma}_{h} \cdot \boldsymbol{n}>_{\partial E \cap \partial \Omega_{N}} \\
= & <\boldsymbol{w}_{h}, \rho^{-1} \boldsymbol{g}>_{\partial E \cap \partial \Omega_{N}}-<\boldsymbol{\tau}_{h}, \boldsymbol{C}:\left(\boldsymbol{n} \otimes_{s} \partial_{t} \tilde{\boldsymbol{u}}_{D}\right)>_{\partial E \cap \partial \Omega_{D}}
\end{aligned}
$$

In (53) the upwind numerical flux terms can be easily calculated by respectively projecting the test field $\boldsymbol{W}_{h}$ and the solution field $\boldsymbol{U}_{h}$ on the right and left eigen bases $\left\{\boldsymbol{R}_{\boldsymbol{n}, k}\right\}$ and $\left\{\boldsymbol{L}_{\boldsymbol{n}, k}\right\}$ of the normal Jacobian operator $\boldsymbol{A}_{\boldsymbol{n}}$ of the element $E$ and also on those ones of its neighboring elements $E^{\prime}$. Each element computes 

and its "inward propagating" fluxes for itself.

The variational formulation (53) is developed for a general Hooke tensor $C$ and works for the general anisotropic case. Indeed, the anisotropy is automatically taken into account by the eigenvalues $\left\{\lambda_{\boldsymbol{n}, k}\right\}$ and the eigenvectors

${ }_{420}\left\{\boldsymbol{R}_{\boldsymbol{n}, k}, \boldsymbol{L}_{\boldsymbol{n}, k}\right\}$, which depend on the $\boldsymbol{n}$ direction indicating in fact how the element interface is oriented regarding the principal directions of anisotropy. Further, 533 works also automatically for heterogeneous cases with discontinuous material properties. Indeed, $\left\{\lambda_{\boldsymbol{n}, k}, \boldsymbol{R}_{\boldsymbol{n}, k}, \boldsymbol{L}_{\boldsymbol{n}, k}\right\}$ are computed with the material properties $(\rho, \boldsymbol{C})$ in $E$, while $\left(\lambda_{\boldsymbol{n}^{\prime}, k}^{\prime}, \boldsymbol{R}_{\boldsymbol{n}^{\prime}, k}^{\prime}, \boldsymbol{L}_{\boldsymbol{n}^{\prime}, k}^{\prime}\right)_{k}$ with the ones $\left(\rho^{\prime}, \boldsymbol{C}^{\prime}\right)$ defined 425 in $E^{\prime}$.

Concerning the two coupled upwind numerical fluxes, the only change in the element-wise dG variational formulation $(53)$ is to calculate the right and left eigenvector bases with the averaged material properties $(\bar{\rho}, \overline{\boldsymbol{C}})$ of $E$ and $E^{\prime}$.

Concerning the two upwind fluxes "G_Flux" and "MG_Flux" developed in the $1 \mathrm{D}$ case, the element-wise variational formulation becomes, for exemple in the case of "MG_Flux":

$$
\begin{aligned}
& \left(\boldsymbol{W}_{h}, \partial_{t} \boldsymbol{U}_{h}\right)_{E}+\left(\boldsymbol{W}_{h}, \boldsymbol{A}^{\partial_{x}}\left(\boldsymbol{U}_{h}\right)\right)_{E} \\
-\quad & <\lambda_{\boldsymbol{n}, k}^{-} \boldsymbol{R}_{\boldsymbol{n}, k}^{-} \cdot \boldsymbol{W}_{h}, \boldsymbol{L}_{\boldsymbol{n}, k}^{-} \cdot \boldsymbol{U}_{h}>_{\partial E_{i n t}}+<\lambda_{\boldsymbol{n}, k}^{-} \boldsymbol{R}_{\boldsymbol{n}, k}^{-} \cdot \boldsymbol{W}_{h}, \boldsymbol{L}_{\boldsymbol{n}, k}^{-} \cdot \boldsymbol{U}_{h}^{\prime}>_{\partial E_{i n t}} \\
+\quad & <\boldsymbol{w}_{h}, \rho^{-1} \boldsymbol{\sigma}_{h} \cdot \boldsymbol{n}>_{\partial E \cap \partial \Omega_{N}} \\
= & <\boldsymbol{w}_{h}, \rho^{-1} \boldsymbol{g}>_{\partial E \cap \partial \Omega_{N}}-<\boldsymbol{\tau}_{h}, \boldsymbol{C}:\left(\boldsymbol{n} \otimes_{s} \partial_{t} \tilde{\boldsymbol{u}}_{D}\right)>_{\partial E \cap \partial \Omega_{D}}
\end{aligned}
$$

The modified left eigenmodes $\sim\left\{\boldsymbol{L}_{\boldsymbol{n}, k}\right\}$ should be calculated taking into account the material properties of the neighbouring elements $E^{\prime}$.

\subsection{Space $d G$ semi discretized formulation}

In the present work, the same finite element basis functions $\psi_{I}^{E}(\boldsymbol{x})$ are used in each element $E$ for the velocity and the stress unknowns and we obtain the following expansions:

$$
\left.\boldsymbol{v}_{h}(\boldsymbol{x}, t)\right|_{E}=\sum_{I, i} V_{I i}^{E}(t) \psi_{I}^{E}(\boldsymbol{x}) \boldsymbol{e}_{i},\left.\boldsymbol{\sigma}_{h}(\boldsymbol{x}, t)\right|_{E}=\sum_{I, i j} S_{I i j}^{E}(t) \psi_{I}^{E}(\boldsymbol{x}) \boldsymbol{e}_{i} \otimes \boldsymbol{e}_{j}
$$

with $i, j=1, \cdots, d$ and $I=1, \cdots, N^{E}, N^{E}$ being the total number of FE basis functions for $E$. If we denote the $\mathrm{FE}$ basis functions of the generalized unknowns ${ }_{440}$ by $\boldsymbol{\Psi}_{p}^{E}$ for $p=I i$ or $I i j$, with $\boldsymbol{\Psi}_{I i}^{E}=\left(\psi_{I}^{E} \boldsymbol{e}_{i} \mathbf{0}\right)^{T}$ and $\boldsymbol{\Psi}_{I i j}^{E}=\left(\mathbf{0} \psi_{I}^{E} \boldsymbol{e}_{i} \otimes \boldsymbol{e}_{j}\right)^{T}$, the expansion of the generalized unknowns in each $E$ reads as:

$$
\left.\boldsymbol{U}_{h}(\boldsymbol{x}, t)\right|_{E}=Y_{p}^{E}(t) \boldsymbol{\Psi}_{p}^{E}(\boldsymbol{x})
$$

with $Y_{I i}=V_{I i}$ and $Y_{I i j}=S_{I i j}$. At last, using the FE expansion (56), we get the following element-wise semi discretized $\mathrm{dG}$ formulation from (53):

$$
[M]^{E E} \cdot\left\{\partial_{t} Y\right\}^{E}+\left([K]^{E E}+\left[K^{f}\right]^{E E}\right) \cdot\{Y\}^{E}+\left[K^{f}\right]^{E E^{\prime}} \cdot\{Y\}^{E^{\prime}}=\{G\}^{E}
$$

with $[M]^{E E}$ the elementary mass matrix, $[K]^{E E}$ the elementary stiffness matrix ${ }_{445}$ (by abuse of terminology), $\left[K^{f}\right]^{E E}$ the elementary flux stiffness matrix contributed by the element $E$ itself, $\left[K^{f}\right]^{E E^{\prime}}$ the elementary flux stiffness matrix 
contributed by the neighboring elements $E^{\prime}$ and $\{G\}^{E}$ the elementary loading vector. The detailed definition of those matrices and vectors are given in Appendix A4. We remark that only the elementary mass matrix $[M]^{E E}$ is symmetric and it is also positive-definite.

By assembling all the elementary contributions, the space dG semi discretized formulation can be written in the following matrix form:

$$
[M] \cdot\left\{\partial_{t} Y\right\}+\left([K]+\left[K^{f}\right]\right) \cdot\{Y\}=\{G\}
$$

where $[M],[K]$ and $\left[K^{f}\right]$ are respectively the global mass, stiffness and flux stiffness matrices, they all have block structure and the sub block matrices are already defined in the element-wise semi discretized formulation (57). Further, both $[M]$ and $[K]$ are block diagonal matrices.

\subsection{Time discretization using the standard four-stage fourth-order $R K$}

Now, we consider the time domain solving of the semi discretized dG formulation (58). The time interval $\mathscr{T}$ is partitioned into $N$ subintervals: $\mathscr{T}=$ $\cup\left[t^{n}, t^{n+1}\right], t^{n+1}=t^{n}+\Delta t$. The FE solution $\{Y\}^{n+1}$ at the time $t^{n+1}$ is computed from the already calculated $\{Y\}^{n}$ at time $t^{n}$. To do that, we use the standard four-stage fourth-order Runge-Kutta iterative method, which calculates four extra stages $\{Z\}^{(k)}(k=1, \cdots, 4)$ to advance from $\{Y\}^{n}$ to $\{Y\}^{n+1}$. More precisely, when it is applied to (58), the following algorithm is obtained:

$$
\begin{aligned}
\{Z\}^{(1)} & =[M]^{-1} \mathscr{L}\left(\{Y\}^{n}\right) \\
\{Z\}^{(2)} & =[M]^{-1} \mathscr{L}\left(\{Y\}^{n}+\frac{1}{2} \Delta t\{Z\}^{(1)}\right) \\
\{Z\}^{(3)} & =[M]^{-1} \mathscr{L}\left(\{Y\}^{n}+\frac{1}{2} \Delta t\{Z\}^{(2)}\right) \\
\{Z\}^{(4)} & =[M]^{-1} \mathscr{L}\left(\{Y\}^{n}+\frac{1}{2} \Delta t\{Z\}^{(3)}\right) \\
\{Y\}^{n+1} & =\{Y\}^{n}+\frac{1}{6} \Delta t\left(\{Z\}^{(1)}+\{Z\}^{(2)}+\{Z\}^{(3)}+\{Z\}^{(4)}\right)
\end{aligned}
$$

465

with $\mathscr{L}(\{Y\})=\{G\}-\left([K]+\left[K^{f}\right]\right) \cdot\{Y\}$.

It is useful to note that in practice the explicit Runge-Kutta algorithm (59) is applied to the element-wise semi discretized formulation (57) since there is no need to assemble the system.

The fourth-order explicit RK scheme is only conditionally stable. According to [14, the time step should satisfy the following stability condition written in the general context of anisotropic and piecewise homogeneous media:

$$
\Delta t \leq \frac{C F L_{s D G}}{2 N_{p}+1} \min _{E}\left\{\frac{h^{E}}{\max _{\boldsymbol{n}}\left\{c_{\boldsymbol{n}, L}^{E}\right\}}\right\}
$$

with $N_{p}$ the order of the FE basis function, $h^{E}$ the size of the element $E$ and $C F L_{s D G}$ the Courant-Friedrichs-Levy number. 
The definition of the element size $h^{E}$ depends on the shortest wavelength of interest, which is defined by the highest frequency of interest. It depends also on the order of $\mathrm{FE}$ basis functions. In the present work, linear elements are used for 1D problems and square bilinear elements for 2D problems. In both cases, $h^{E}$ is chosen so that there are at least about 20 elements in the shortest wavelength of longitudinal waves. The definition of highest frequency of interest will be presented in the next section.

The constant $C F L_{s D G}$ depends on the order of the $\mathrm{FE}$ basis function, on the space dimension $d(d=1,2,3)$ as well as on the shape of the elements. In the present work, $C F L_{s D G}$ is taken equal to about 0.9 in the $1 \mathrm{D}$ case and to about 0.6 in the $2 \mathrm{D}$ case.

\section{Numerical investigations of the numerical fluxes}

As previously indicated, when the elastic moduli and the density of a propagating medium are continuous, all the numerical fluxes considered in the present work are identical, are the exact solution of the Riemann problem defined at element interfaces and give rise to physically coherent interface conditions. This 490 is no longer true when physical interfaces exist. The first goal of this section is to investigate the behavior of the upwind numerical fluxes with respect to the degree of inhomogeneity in elastic moduli in simpler cases of $1 \mathrm{D}$ or $2 \mathrm{D}$ domains composed of two different materials. In a second time, the behaviors of the two coupled fluxes in the case of piecewise homogeneous media including a large number of physical interfaces, such as 2D polycrystalline media, are studied.

Each considered numerical flux gives rise to a space dG solver. They are named hereafter "k_sDG", "u_sDG", "cV_sDG", "cR_sDG", "G_sDG" and "MG_sDG" corresponding respectively to the flux proposed by Käser et al., the uncoupled flux "u_Flux", the coupled flux "cV_Flux" with the Voigt average $C^{\text {Voigt }}$, the coupled flux "cR_Flux" with the Reuss average $C^{\text {Reuss }}$, and the two upwind fluxes "G_Flux" and "MG_sDG" developed in the 1D case. Some analytical solutions, such as reflection and transmission coefficients or wave phase velocities, are used when available to analyze the quality of the numerical fluxes.

To obtain reference numerical solutions used for comparison, two dynamic solvers using continuous finite elements in the space have been used: the implicit Newmark method with $(\gamma, \beta)=\left(\frac{1}{2}, \frac{1}{4}\right)$ and a time discontinuous space-time Galerkin method. As the two solvers give substantially identical solutions, only the solutions calculated by the time discontinuous space-time Galerkin method, named hereafter "tDG", are presented.

We recall that the basic idea of the time discontinuous space-time Galerkin method is to subdivide the studied space-time domain $\Omega \times] 0, T$ [ into a series of space-time slabs $\Omega \times] t_{n}, t_{n+1}$ [ and to write in each space-time slab a variational formulation for the displacement and velocity fields simultaneously in space and in time. Within each space-time slab, continuous finite elements are used, but between two successive slabs, both displacement and velocity fields are discontinuous [15 18. When space-time $\mathrm{FE}$ basis functions are chosen equal to the product of $\mathrm{FE}$ basis functions in space and $\mathrm{FE}$ basis functions in time, 
it can be shown that time $\mathrm{dG}$ methods often lead to unconditionally stable implicit time-stepping schemes. The numerical damping inherent to the time $\mathrm{dG}$ methods, due to energies dissipated in time jumps between two subsequent space-time slabs, increases with the frequency and allows filtering numerical spurious noises. The time $\mathrm{dG}$ solver used in this work discretizes the space with a finite element mesh combined with one linear element in time for each space-time slab. It has been validated by our previous studies [18 20] and also ${ }_{525}$ by other authors [17, 21].

Regarding the choice of the element size in space, the strategy defined in Section 3.5 is adopted for all the solvers. As for the definition of the time step for the time dG solver "tDG", it is necessary to take a sufficiently small time step to prevent higher frequency modes of interest from numerical damping, even if the solver "tDG" is unconditionally stable and does not suffer from instability concern. Consequently, the following condition is applied with the constant $C_{t D G-\text { damping }}$ taken equal to about 1.0 according to our numerical experiences:

$$
\Delta t \leq C_{t D G-\text { damping }} \min _{E}\left\{\frac{h^{E}}{\max _{\boldsymbol{n}}\left\{c_{\boldsymbol{n}, L}^{E}\right\}}\right\}
$$

Generally, the time step for the solver "tDG" can be taken larger than the one for the solvers "sDG" for a same element size.

For all the numerical examples presented hereafter, the dependency of the external loadings upon the time $t$ is always chosen to be a ricker signal, i.e.:

$$
\boldsymbol{g}(\boldsymbol{x}, t)=\boldsymbol{a}_{g}(\boldsymbol{x})\left(1-2\left(\frac{2 \pi\left(t-T_{r} / 2\right)}{T_{r}}\right)^{2}\right) e^{-\left(\frac{2 \pi\left(t-T_{r} / 2\right)}{T_{r}}\right)^{2}}
$$

with $T_{r}$ the period of the ricker signal. This choice is motivated by the fact that ricker signals provide a perfectly controlled frequency content, whose cen540 tered frequency is $f_{\max }=2 T_{r}^{-1}$ and whose cutoff frequency can be reasonably considered as $f_{c}=2.5 f_{\max }$. Figure 2 shows a normalized ricker signal and its normalized frequency content. As indicated in the previous section, for all the numerical simulations presented in the present section, the highest frequency of interest is chosen equal to $f_{c}$ and the associated shortest wavelength is used to define the element size $h^{E}$.

In the following, the orthonormal bases of $1 \mathrm{D}$ and $2 \mathrm{D}$ spaces are respectively denoted by $\left(\boldsymbol{e}_{x}\right)$ and $\left(\boldsymbol{e}_{x}, \boldsymbol{e}_{y}\right)$.

\subsection{Reflection and transmission behaviors in the $1 D$ case}

A $1 D$ elastic rod $\Omega=\Omega_{1} \cup \Omega_{2}$ composed of two materials is studied and two types of discontinuities are considered (Figure 3):

- Case 1 (Figure 3(a)). For this case, the density $\rho=2500 \mathrm{~kg} \cdot \mathrm{m}^{-3}$ is uniform in the whole rod while the Young's modulus in the two subdomains is respectively $E_{1}=22.5 \mathrm{GPa}$ and $E_{2}=a^{2} E_{1}$. The phase velocity in the two subdomains is respectively equal to $c_{L 1}=3000 \mathrm{~m} \cdot \mathrm{s}^{-1}$ and $c_{L 2}=a c_{L 1}$. The total length of the rod is $L=L_{1}+L_{2}=(1+a) L_{1}$, with $L_{1}=900 \mathrm{~m}$. 
- Case 2 (Figure 3(b)). For this case, the density and the Young's modulus in the two subdomains are respectively $\left(\rho=2500 \mathrm{~kg} \cdot \mathrm{m}^{-3}, E_{1}=22.5 \mathrm{GPa}\right)$ and $\left(\rho_{2}, E_{2}\right)=\left(a \rho_{1}, a E_{1}\right)$. The phase velocity in the two subdomains is the same $c_{L 1}=c_{L 2}=3000 \mathrm{~m} \cdot \mathrm{s}^{-1}$. The total length of the rod is $L=L_{1}+L_{2}$, with $L_{1}=L_{2}=900 \mathrm{~m}$.

For both cases, the impedance contrast between the two subdomains is $\sqrt{E_{2} \rho_{2}} /$ $\sqrt{E_{1} \rho_{1}}=a$.

A pressure loading $g(t)$ is applied on the left end and the free boundary condition is prescribed on the right end. $g(t)$ is a ricker signal with period $T_{r}=$ $0.04 \mathrm{~s}$ and cutoff frequency $f_{c}=125 \mathrm{~Hz}$. The corresponding shortest wavelength to consider is $\ell_{L}\left(f_{c}\right)=24 \mathrm{~m}$. The time that waves need to propagate from the left end to the physical interface is equal to $0.2 \mathrm{~s}$, so is the time from the physical interface to the right end. According to the previously presented strategy, we have the following element sizes in the two subdomains: $h_{1}^{E}=0.6 \mathrm{~m}$ and $h_{2}^{E}=$ ${ }_{570} a h_{1}^{E}$ for the case $1 ; h_{1}^{E}=h_{2}^{E}=0.6 \mathrm{~m}$ for the case 2 . This choice results in 40 elements in the shortest longitudinal wavelength of interest. The time step $\Delta t=62.5 \mu \mathrm{s}$ is chosen according to (60) and is used also for the solver "tDG", which leads to $C F L_{s D G}=0.94$ and $C_{t D G-\text { damping }}=0.31$.

We recall that the reflection and transmission coefficients $U_{R}$ and $U_{T}$ are analytically known in the $1 \mathrm{D}$ case:

$$
U_{R}=\frac{1-a}{1+a}, U_{T}=\frac{2}{1+a}
$$

The parameter $a$ measures the degree of inhomogeneity of the physical interface. Different values of $a \in(0.9,0.8,0.5,0.25,0.2,0.15,0.1,0.05)$ are considered to study the quality of the six numerical fluxes as a function of the degree of inhomogeneity.

The Case 1 (Figure 3(a)), where only the Young's modulus is discontinuous, is mainly studied. The first result is that both uncoupled flux "u_Flux" and the flux "G_Flux" resulting in mechanically incoherent interface conditions fail to properly represent the wave reflection and transmission at the physical interface. Indeed, Figure 4 compares the velocity field $v(x)$ at four instants ${ }_{585} t=0.1,0.2,0.3$ and 0.4 s solved by the solvers "u_sDG", "G_sDG" and "tDG" in the case of $a=0.5$. The results obtained by the other solvers "k_sDG", "cV_sDG", "cR_sDG" and "MG_sDG" are not presented as they give nearly the same results than those obtained by the solver "tDG". We recall that the wave front arrives at the physical interface at $t=0.2 \mathrm{~s}$. It can be noticed that, after the waves have passed through the physical interface, the results obtained by both solvers " $\mathrm{u}_{-} \mathrm{sDG}$ " and "G_sDG" completely differ from those obtained by the solver "tDG": the reflected wave front has a negative amplitude while it should be positive and the amplitude of the transmitted wave front is largely overestimated. We notice that this behavior remains unchanged even if the 595 degree of inhomogeneity is low, that is when $a \rightarrow 1$. Therefore, both solvers "u_sDG" and "G_sDG" are not considered in the following studies.

As another important result, all the space dG solvers using a numerical flux that does not exactly solve the Riemann problem encounter the instability 
problem when the degree of inhomogeneity increases, whilst for both solvers "G_sDG" and "MG_sDG" no instability problem is observed and the same stability condition applies regardless of the degree of inhomogeneity. For the two solvers using the coupled fluxes "cV_sDG" and "cR_sDG", the time step should be divided by 2 for $a=0.15,0.1$ and by 4 for $a=0.05$. For the two solvers "k_sDG" and "u_sDG", the instability phenomenon appears with a higher degree of inhomogeneity $a=0.01$ and the time step should be divided by 4 .

To assess the quality of each solver to represent the physical interface, relative errors $e_{R}$ and $e_{T}$ for the reflection and transmission coefficients calculated in the following way are considered:

$$
e_{R}=\frac{\left|U_{R, h}-U_{R}\right|}{\left|U_{R}\right|}, e_{T}=\frac{\left|U_{T, h}-U_{T}\right|}{\left|U_{T}\right|}
$$

where $U_{R}$ and $U_{T}$ are the analytical coefficients given by $(63)$ and $U_{R, h}$ and $U_{T, h}$ are their numerically calculated values.

It is worth noticing that the relative errors calculated in this way include also discretization errors (including the treatment of boundary conditions), which depend on the element size and type and on the time integration procedure. To quantify the contribution of sources of errors other than the treatment of physi615 cal interfaces, the case with $a=1.0$ is also computed, which corresponds to the homogeneous case without physical interfaces. In this way, the accumulation of error with propagation distance in the homogeneous part of the studied domain is quantified. We note that for $a=1.0$ only the relative error of the transmission coefficient can be obtained, as $U_{R}=0$.

The relative errors (64) are firstly calculated after only one crossing through the interface, $U_{R, h}$ and $U_{T, h}$ being calculated as the ratio of maximum amplitudes between the reflected and transmitted wave fronts recorded at $t=0.3$ and the initial wave front recorded at $t=0.1 \mathrm{~s}$. Between those two instants, the distance waves have propagated is equal to 24 times the shortest wavelength of interest. Figure 5(a) shows the evolution of the relative errors for all solvers as a function of the parameter $a$. It can be noticed that the errors of the solver "tDG" do not depend on the parameter $a$ and there is no difference in the errors in the reflection and transmission coefficients. When the Riemann problem on physical interfaces is defined in a relevant way and exactly resolved, the so${ }_{630}$ defined upwind flux leads to a solver that behaves in the same way. Indeed, the errors obtained by the solver "MG_sDG" do not depend on the parameter $a$ and both reflection and transmission errors are equal and are furthermore slightly smaller than those obtained by the solver "tDG". On the other hand, the errors obtained by the space dG solvers "k_sDG", "cV_sDG" and "cR_sDG" increase when $a$ decreases. The error in the transmission coefficient are larger than the one in the reflected coefficient and it increases rapidly for the solver "cR_sDG" when $a \rightarrow 0$. For $a \geq 0.5$, all the errors remain inferior to $0.15 \%$. Furthermore, for $a \rightarrow 1$, all measured errors decrease and reach a plateau, the value of the plateau is the one obtained in the homogeneous domain (with $a=1$ ), which 640 should be controlled by the discretization parameters in space and in time. 
When we consider their long-term behavior, the relative errors are calculated for eight successive instants from $t=0.3 \mathrm{~s}$ to $t=5.9 \mathrm{~s}$. The instant $t=5.9 \mathrm{~s}$ corresponds to a propagation distance equal to about 730 times the shortest wavelength of interest. Figures 6(a) and (b) show that the relative errors of the space dG solvers "k_sDG", "cV_sDG" and "MG_sDG" increase linearly with $N_{\text {interface }}$ the number of interface crossings. Comparing with the curves obtained with $a=1.0$, we can conclude that in the accumulation of error for both solvers "k_sDG" and "cV_sDG", there is a part that is due to the inaccuracy of the numerical flux to represent the physical interface. This part increases with ${ }_{650}$ the increase of the degree of inhomogeneity. Once more, it is found that the accuracy of the solver "MG_sDG" is independent on the degree of inhomogeneity. Indeed, a unique curve is observed for both reflection and transmission errors regardless of the parameter $a$ (Figures 6(b)). It can be concluded that there is only the accumulation of discretization errors. The same conclusion is made for the reference solver "tDG". However, the discretization errors of the solver "tDG" increase more rapidly than those of the solver "MG_sDG".

Reflected wave fronts calculated at different instants by the two solvers "cV_sDG" and "tDG" are plotted in Figure 7 in the case of $a=0.25$. They are much better captured by the solver "cV_sDG" than the solver "tDG". The numerical results of the solvers "k_sDG", "cR_sDG" and "MG_sDG" are not presented as they are nearly identical to those obtained by "cV_sDG".

The Case 2 (Figure 3(b)) is considered as the type of discontinuity, discontinuities only in $E$ or in both $E$ and $\rho$, has effects on the arithmetic and harmonic averages used to define the coupled fluxes. For this case, the same results are obtained for the solver "MG_sDG". Namely, there is no worsening of the stability condition when the degree of inhomogeneity increases, the errors in both reflection and transmission coefficients are the same and do not depend neither on the degree of inhomogeneity nor on the type of discontinuities (Figure 5(b)). As for the solver "k_sDG", the same errors are obtained as in the Case 1. It can 670 be concluded that the behavior of the solver "k_sDG" does not depend on the type of discontinuity. Concerning the two solvers with the coupled numerical fluxes "cV_sDG" and "cR_sDG", unlike in the Case 1 of discontinuous Young's modulus, the errors in the transmission coefficient are similar, while the error in the reflection coefficient of "cV_sDG" increases more rapidly with the degree of inhomogeneity than "cR_sDG". For $a \geq 0.05$, all relative errors for the three solvers "k_sDG", "cV_sDG" and "cR_sDG" remain smaller than $0.33 \%$.

In summary, we get the following conclusions in the $1 \mathrm{D}$ case:

- For the space dG solvers, neither "u_sDG" with the uncoupled numerical flux nor "G_sDG" with the upwind flux that are exact solutions of the Riemann problem with the mechanically incoherent interface conditions $35 \mathrm{~b}$ ) (see also (43p) works. The solver "MG_sDG" with the upwind flux that are exact solutions of the Riemann problem with the classical mechanical interface conditions 45b (see also (44)) works perfectly. Its accuracy, its long-term behavior and its stability condition are independent of the degree of inhomogeneity and of the type of discontinuities. 
- All the space dG solvers using a numerical flux that does not exactly solve the Riemann problem, "k_sDG", "u_sDG", "cV_sDG" and "cR_sDG", can encounter instability problems. Indeed, the stability condition is more and more worsened when the degree of inhomogeneity increases, that is $a \rightarrow 0$. The precise mechanism of this worsening has not yet been well understood. However, as for the fluxes "G_sDG" and "MG_sDG" the stability condition is not altered regardless the degree of inhomogeneity, we believe that the fact that the Riemann problem is not exactly solved by the simplified numerical fluxes should be the main cause, if not the only.

- For $a \geq 0.25$, both coupled numerical fluxes give similar results than the one proposed by Käser et al. When $a \rightarrow 0$, errors obtained by the Reuss average coupled numerical flux "cR_sDG" increase rapidly in the case of discontinuous Young's modulus. For $a \geq 0.5$, both coupled fluxes and the one proposed by Käser et al. are interesting as they give rise to an error in the reflection coefficient smaller than the one obtained by the flux "MG_sDG", while the errors in the transmission coefficient obtained by all the four fluxes are close.

- The long-term behavior of the space dG solvers "MG_sDG" (for all $a$ ), "k_sDG" and "cV_sDG" (for $a \geq 0.25$ ) is much better than the reference solver "tDG", even when there is no physical interface. We believe that is because the space discontinuous elements with appropriate numerical fluxes are more accurate than the continuous one to capture waves, all other things being equal. The $H^{1}$-classe finite element methods seem more appropriate to deal with the wave propagation problems, as they provide continuous displacement and velocity solutions. However, as they use piecewise polynomial basis functions for the displacement field that is the primary unknown field, the corresponding stress vector fields are discontinuous between elements and no treatment specific to the hyperbolic characters of the wave propagation problem is involved within the space continuous weak formulation, which leads to numerical dispersion errors. With the use of numerical fluxes that directly take into account wave propagation velocities and modes, the space discontinuous finite element methods give a better control to such errors, even if both velocity and stress solution fields are discontinuous.

\subsection{Reflection and transmission behaviors in the $2 D$ bimaterial case}

The first 2D example considers a $2 \mathrm{D}$ rectangular elastic domain $\Omega=\Omega_{1} \cup \Omega_{2}$ with two vertical interfaces. Both materials are isotropic and have the same density $\rho=4428 \mathrm{~kg} \cdot \mathrm{m}^{-3}$. The dimensions and the material properties are indi725 cated in Figure 8. The width of the whole domain is $L_{x}=20.32 \mathrm{~mm}$ and the Hooke tensor $\boldsymbol{C}_{1}$ of the softer material at the center is the same as the reference Voigt average Hooke tensor $\boldsymbol{C}^{r e f-V o i g t}$ of the polycrystal considered in the next section, their values are presented in Table I. The corresponding Young's moduli of both materials are $E_{1}=73.95 \mathrm{GPa}$ and $E_{2}=(1.5)^{2} E_{1}$ and they have 

and the transverse waves are respectively $c_{L 1}=5879 \mathrm{~m} \cdot \mathrm{s}^{-1}, c_{T 1}=2446 \mathrm{~m} . \mathrm{s}^{-1}$, $c_{L 2}=1.5 c_{L 1}=8819 \mathrm{~m} . \mathrm{s}^{-1}, c_{T 2}=1.5 c_{T 1}=3670 \mathrm{~m} . \mathrm{s}^{-1}$. In terms of degree of inhomogeneity, measured by the parameter $a$ defined for the previous 1D example, we have $a=1 / 1.5=0.67$ for the present example. extremely short segment $L_{e}$ of length $50 \mu \mathrm{m}$ at the center of the external top boundary of $\Omega$, while free surface boundary conditions are prescribed on the remaining parts. The pressure $g(x, t) \boldsymbol{e}_{y}$ is uniformly distributed in space and its period of ricker signal in time is $T_{r}=0.64 \mu \mathrm{s}$, resulting in $f_{\max }=3.1 \mathrm{MHz}$ and $f_{c}=7.8 \mathrm{MHz}$. According to the setting of the numerical model, the $O y$ axis is in fact a line of symmetry of the problem.

The space dG solvers using different numerical fluxes "k_sDG", "u_sDG", "cV_sDG" and "cR_sDG" are applied to the 2D example and are compared to the reference solver "tDG". Concerning the numerical flux "MG_sDG", which solves exactly the Riemann problem, its implementation is currently verified in both 2D and 3D cases and is therefore not considered hereafter.

Bilinear square finite elements of size $h=25 \mu \mathrm{m}$ are used to discretize the whole domain. This choice corresponds to respectively 30 and 45 elements in the shortest longitudinal wavelengths of interest $\ell_{L 1}\left(f_{c}\right)=0.75 \mathrm{~mm}$ in $\Omega_{1}$ and ${ }_{750} \ell_{L 2}\left(f_{c}\right)=1.1 \mathrm{~mm} \Omega_{2}$. The time step used for the space $\mathrm{dG}$ solvers $\Delta t_{s D G}$ is calculated using the maximum longitudinal wave $c_{L 2}$ and is equal to $0.533 \mathrm{~ns}$, resulting in $C F L_{s D G}=0.56$ (see 600 ). As in the case of the $1 \mathrm{D}$ example, this time step is also used for the implicit solver "tDG". Concerning the total analysis time, it is equal to $4 \mu$ s so that main wave fronts reflected by the bottom boundary of the rectangular domain have time to arrive at the top boundary.

The present $2 \mathrm{D}$ problem is slightly different to the two quarter-spaces problem presented in 22 due to its additional symmetric setting and free boundary conditions, which reflect wave back into the domain. However, the main phenomena analyzed in 22] remain unchanged. As suggested in 22, seismograms 760 recorded along the external top horizontal boundary and the internal veritical physical interface on the right are displayed respectively in Figures 9 and 10 Only the seismograms obtained by the solver "cV_sDG" are presented as those obtained by the other solvers "k_sDG", "cR_sDG" and "tDG" are identical. The main phenomena of reflection, transmission and conversion of the longitudinal 765 and Rayleigh waves are shown in Figure 9. We remark that the amplitudes of reflected $\mathrm{L}$ waves are too small to be noticeable in the seismograms. Figure 10 presents another main phenomenon, that is an interface wave generated from the incident Rayleigh and propagating downwards along the vertical physical interface. It will be shown by the following quantitative analyses that the interface wave propagates with a velocity equal approximately to $c_{I}=3440 \mathrm{~m} . \mathrm{s}^{-1}$.

Concerning the space dG solver using the uncoupled numerical flux "u_sDG", it gives rise to important differences in velocity components when compared to the other solvers, as shown in Figure 11. This confirms the conclusion made in the $1 \mathrm{D}$ case. Nevertheless, the phenomenon of wavefront inversion, i.e. positive 775 amplitudes become negative, is not observed as in the case 1D, which means 
that only a qualitative comparison of wavefronts would not be capable to reveal the problem. As for the space dG solvers "k_sDG", "cV_sDG" and "cR_sDG", it has been verified using the seismograms that they allow correct captures of all the wave phenomena triggered at the physical interface. However, it is necessary to perform quantitative comparison with the reference solver "tDG".

The different wave fronts captured by the solvers "tDG", "k_sDG", "cV_sDG" and "cR_sDG" are compared at first. The wave fronts are identified by searching local maximum and minimum amplitude using an automatic procedure. Markers indicating the local maximum and minimum points are displayed in Figures 9 and 10. They are gathered and compared in Figure 12. The three space dG solvers "k_sDG", "cV_sDG" and "cR_sDG" give identical results, which is coherent with the previous $1 \mathrm{D}$ analyses as the degree of inhomogeneity chosen for the $2 \mathrm{D}$ example is low corresponding to $a=0.67$. Compared to the results obtained by "tDG", differences are nearly negligible.

Then, more quantitative analyses are presented in Figures 13 and 14 . For the sake of clarity, the curves obtained by the solver "cR_sDG" are not presented. Figure 13 compares maximum and minimum amplitudes of different wave fronts during their propagation calculated by the different solvers and shows that the curves calculated by the different solvers are very close. Figure 14 considers the 795 accuracy of the phase velocity estimated by the different solvers. The comparison is made in terms of a relative error, which is defined in the following way:

$$
e_{r, j}=\frac{c_{n u m, j}-c_{j}}{c_{j}}
$$

where $j \in\{L, R$, reflR, $I\}$ for different types of wave, reflR indicating the reflected $\mathrm{R}$ wave for which we define $c_{\text {reflR }}=-c_{R}$. Numerically estimated phase velocities $c_{n u m, j}$ are obtained by calculating the slopes of the numerically identified wave fronts. We recall that in an isotropic elastic material, the Rayleigh wave velocity can be expressed as a function of the Poisson's ratio $\nu$ and the transverse wave velocity $c_{T}$ as follows:

$$
c_{R}=\frac{0.862+1.14 \nu}{1+\nu} c_{T}
$$

We have, for the present numerical example, $\left(c_{R 1}, c_{R 2}\right)=0.94\left(c_{T 1}, c_{T 2}\right)$. As there is no analytical solution of the velocity of the interface wave, an approximately estimated value $c_{I}=1.125\left(c_{T 1}+c_{T 2}\right) / 2=3440 \mathrm{~m} \cdot \mathrm{s}^{-1}$ is used. We notice that, far from boundaries, interfaces or meeting points of two waves, where the estimation of phase velocities is not easy, the relative errors presented in Figure 14 are small. The worst result is obtained for the interface wave but the ${ }_{810}$ errors still remain smaller than $10 \%$. The best result are obtained for the incident and transmitted Rayleigh waves, which are the waves propagating the most energy in the studied problem.

In summary, the conclusions made in the 1D case are confirmed by the study of the $2 \mathrm{D}$ bimaterial problem. The uncoupled numerical flux fails to correctly 815 represent wave propagation phenomena at a physical interface, even in the case 
of a relatively low degree of inhomogeneity in terms of wave velocities $(a=0.67)$. Both coupled numerical fluxes work well in this case as they give quantitatively good comparisons with the reference solvers.

\subsection{Upwind numerical fluxes applied to polycrystalline materials}

The considered space dG solvers using the proposed coupled fluxes, "cV_sDG" and "cR_sDG", are applied to single-phase and untextured polycrystalline materials. A single-phased polycrystal is an assembly of small single crystals bonded together. Each crystallite (grain) is anisotropic and they all have the same elastic Hooke tensor but with differently and randomly oriented basis of anisotropy.

225 Such a polycrystalline material is a heterogeneous medium with piecewise homogeneous elastic moduli, which are discontinuous at grain interfaces, elastic waves propagating in it are scattered and attenuated due to interactions between waves and grain interfaces.

In a single-phase polycrystalline material, the degree of inhomogeneity in elastic properties is completely determined by the degree of anisotropy of the crystallite's Hooke tensor: the stronger the anisotropy, the higher the degree of inhomogeneity. In the related literature, the degree of inhomogeneity of such a polycrystal is usually expressed by measuring its departure from its Voigt average homogeneous equivalent medium in the following way [23]:

$$
\xi_{i j k l}^{2}=\frac{1}{4} \frac{<\left(C_{i j k l}(\theta)-C_{i j k l}^{\text {Voigt }}\right)^{2}>_{\theta}}{\left(C_{i j k l}^{r e f-\text { Voigt }}\right)^{2}}
$$

where the subscript "ijkl" has the proper value for either longitudinal or transverse waves and $<\cdot>_{\theta}$ stands for the average over all orientations of the local material anisotropic axes denoted by $\theta$. Furthermore, in order to remain consistent with the previously presented analyses, we also keep measuring the dispersion in the phase velocity of quasi-longitudinal waves with the parameter $a$ defined as follows:

$$
a=\frac{\min _{\theta}\left\{c_{q L}\right\}}{\max _{\theta}\left\{c_{q L}\right\}}
$$

A 2D finite element model for rectangular polycrystals composed of 2106 elliptic grains of size $480 \mu m \times 240 \mu \mathrm{m}$ is defined (Figure 15(a)). The dimensions of the rectangular domain and the boundary conditions are exactly the same as those of the 2D example of the previous section, except that the emitter segment ${ }_{845} L_{e}$ of pressure loading is longer with a length equal to $2.8 \mathrm{~mm}$. Concerning the external pressure loadings, a Gaussian distribution along the emitter segment is chosen for the amplitude of the pressure loading. This is to reduce as much as possible the generation of transverse waves, which would be triggered when discontinuities in boundary conditions occur at the ends of the emitter segment. The period of ricker signal in time of the pressure loadings is $T_{r}=0.4 \mu \mathrm{s}$, resulting in $f_{\max }=5 \mathrm{MHz}$ and $f_{c}=12.5 \mathrm{MHz}$.

Two polycrystals with different degrees of inhomogeneity are considered: 
(1) Reference material denoted by "ref": it is a titanium alloy with crystallites of cubic symmetry. It is orthotropic and its Hooke tensor is denoted by $C^{\text {ref }}$

(2) Material denoted by "strong": to define an orthotropic material with a stronger degree of anisotropy, the Hooke tensor $\boldsymbol{C}^{\text {strong }}$ of a fiber-reinforced material is used and results in very high degree of anisotropy.

For both materials, their homogenized equivalent media are isotropic and the corresponding Voigt average Hooke tensors are respectively denoted by $\boldsymbol{C}^{\text {ref-Voigt }}$ and $\boldsymbol{C}^{\text {strong-Voigt }}$. The elastic moduli in the local material anisotropic basis $\left(\boldsymbol{a}_{1}, \boldsymbol{a}_{2}, \boldsymbol{a}_{3}\right)$ of both polycrystals are given in Table I. as well as the parameters $\xi_{L}$ and $a$ measuring the degree of inhomogeneity of each material. Their densities are $\rho^{\text {ref }}=4428 \mathrm{~kg} \cdot \mathrm{m}^{-3}$ and $\rho^{\text {strong }}=2710 \mathrm{~kg} \cdot \mathrm{m}^{-3}$. Wave velocities in the local material anisotropic plane $\left(\boldsymbol{a}_{1}, \boldsymbol{a}_{2}\right)$ of quasi-longitudinal and quasitransverse waves are presented in Figure 16 and show the dependency of wave velocities on space directions. The high degree of anisotropy of the material "strong" is coherent with the values of $\xi_{L}$ and $a$ shown in Table I]

\begin{tabular}{|c|c|c|c|c|c|c|c|c|c|c|}
\hline \multirow[b]{2}{*}{$\boldsymbol{C}^{r e f}(\mathrm{GPa})$} & & $C_{i i i i}$ & $C_{i i j j, i \neq j}$ & \multicolumn{2}{|c|}{$C_{i j i j, i \neq j}$} & $\xi_{L}$ & $a$ & & & \\
\hline & \multicolumn{2}{|c|}{134.0} & 110.0 & \multicolumn{2}{|c|}{36.0} & 0.028 & 0.899 & & & \\
\hline \multicolumn{2}{|c|}{$C^{\text {ref-Voigt }}(\mathrm{GPa})$} & 153. & 100.0 & \multicolumn{2}{|c|}{26.5} & 0 & 1 & & & \\
\hline & \multicolumn{2}{|c|}{$C_{1111}$} & $C_{i i i i, i=2,3}$ & $\bar{C}_{2233}$ & \multicolumn{2}{|c|}{$C_{11 i i, i=2,3}$} & $C_{1 i 1 i, i=2,3}$ & $C_{2323}$ & $\xi_{L}$ & $a$ \\
\hline$C^{\text {strong }}(\mathrm{GPa})$ & \multicolumn{2}{|c|}{132.5} & 10.3 & 0.4 & \multicolumn{2}{|r|}{3.6} & 4.0 & 5.0 & 0.563 & 0.266 \\
\hline$C^{\text {strong-Voigt }}(\mathrm{GPa})$ & 35. & & 35.4 & 10.6 & & 10.6 & 12.4 & 12.4 & 0 & 1 \\
\hline
\end{tabular}

Table I: Elastic moduli and degrees of inhomogeneity of the studied materials "ref" and "strong", and their homogenized equivalent media "ref-Voigt" and "strong-Voigt"

With randomly distributed crystallographic orientations, wave velocities in the global plane of study $\left(\boldsymbol{e}_{x}, \boldsymbol{e}_{y}\right)$ are different from grain to grain. Ten samples with different random distributions $\left\{\Theta_{i}\right\}_{i=0, \cdots, 9}$ of crystallographic orientations are considered. To present the crystallographic texture of the studied polycristals, Figure 15 displays, in the case of the random distribution $\Theta_{0}$, dispersions $d V / V_{m}$ in the phase wave velocity $c_{q L}(\boldsymbol{k})$ with $\boldsymbol{k}=\boldsymbol{e}_{y}, V_{m}$ being the ${ }_{875}$ averaged wave velocity $\left\langle c_{q L}(\boldsymbol{k})>_{\text {grains }}\right.$ over all grains and $d V=c_{q L}(\boldsymbol{k})-V_{m}$ for each grain.

As for the choice of numerical parameters, square finite elements of size $h=$ $25 \mu \mathrm{m}$ are used for all numerical simulations of the present section and leads to have at least about 19 elements in the shortest quasi-longitudinal wavelength for 880 the cut-off frequency $f_{c}$ for all the studied materials, except the material "strongVoigt" for which the number is about 12. As the principle results considered are numerical measures of the attenuation coefficient and of the noise levels of the quasi-longitudinal waves, the finite element size is defined with respect to the shortest quasi-longitudinal wavelength, which is approximately twice the 885 shortest quasi-transverse wavelength (Figure 16). We note that the influence of the finite element size on these numerical measures has been studied in $[25$, 
27. Table II gives the time step used for each material and each solver, and the associated parameters $C F L_{s D G}$ and $C_{t D G \text {-damping. Concerning reference }}$ numerical solutions, only numerical results obtained with the time $\mathrm{dG}$ solver "tDG" are considered hereafter.

\begin{tabular}{|c|c|c|c|c|}
\hline & "ref" & "ref-Voigt" & "strong" & "strong-Voigt" \\
\hline$c_{q L, \max }\left({\mathrm{m} . \mathrm{s}^{-1}}^{-1}\right)$ & 6122.8 & 5884.2 & 6991.6 & 3612.2 \\
\hline$\ell_{q L, \min }(\mu \mathrm{m})$ & 489 & 471 & 559 & 289 \\
\hline$\ell_{q L, \min } / h^{E}$ & 20 & 19 & 22 & 12 \\
\hline$\Delta t_{s D G}(\mathrm{~ns})$ & 0.89 & 0.89 & 0.78 & 0.78 \\
\hline$C F L_{s D G}$ & 0.65 & 0.63 & 0.65 & 0.33 \\
\hline$\Delta t_{t D G}(\mathrm{~ns})$ & 4 & 4 & 4 & 4 \\
\hline$C_{t D G-\text { damping }}$ & 0.98 & 0.94 & 1.12 & 0.58 \\
\hline
\end{tabular}

Table II: Wave velocities, shortest longitudinal wavelengths, time steps and values of $C F L$ for the studied materials "ref" and "strong", and their homogenized equivalent media "ref-Voigt" and "strong-Voigt"

890

As a first result, numerical solutions show that the uncoupled numerical flux does not work in either cases. Indeed, instability phenomena are encountered by the solver "u_sDG" as shown in Figure 17(a). This confirms the previously obtained conclusion. The second important result is that the Reuss average 895 numerical flux does not work when the degree of inhomogeneity is high. Figure 17 (b) shows the same instability phenomena are encountered by the solver "cR_sDG" in the polycrystal "strong". It seems that the worsening of the stability condition encountered in the previously considered 1D examples is greatly aggravated by the large number of physical interfaces, the instability problem remains even a very small time step is used.

Now, more quantitative comparisons between the two solvers "cV_sDG" and "tDG" are presented. Numerical time-series signals are recorded at 22 probes, which are uniformly distributed on the emitter segment $L_{e}$. Numerical evaluations of the attenuation and the backscattered noise levels in polycrystals by both solvers are compared.

In a polycrystal, the attenuation measures the amplitude decay of elastic waves during their propagation and it can be caused by dissipation, geometrical spreading or scattering-induced diffusion. Since in the application of NDE by ultrasounds, the dissipation by energy transformation into heat due to dis910 locations and the damping is generally negligible, only the scattering-induced attenuation is considered herein and it is usually quantified by a scalar $\alpha$ called the attenuation coefficient, which defines an exponential decay law. Numerical calculation of $\alpha$ in the frequency domain is done in the following way 24, 25): Discrete Fourier Transform is used to decompose the time-series signal of the reflected wave fronts $v_{y}^{r}\left(\boldsymbol{x}_{j}, t\right)$ at the $j$ th probe $\boldsymbol{x}_{j}$ into the frequency domain and gives rise to the corresponding amplitude spectrum $\hat{v}_{y}^{r}\left(\boldsymbol{x}_{j}, f\right)$. Using those data, the attenuation coefficient $\alpha(f)$ as a function of the frequency $f$ is measured as: 


$$
\alpha(f)=-\frac{10}{D} \ln \left(\frac{\sum_{j=1}^{M}\left|\hat{v}_{y}^{r}\left(\boldsymbol{x}_{j}, f\right)\right|^{2}}{\sum_{j=1}^{M}\left|\hat{v}_{y}^{r, r e f}\left(\boldsymbol{x}_{j}, f\right)\right|^{2}}\right)
$$

To eliminate the attenuation due to the geometrical spreading of wavefront, the

920 recorded in the equivalent homogeneous medium instead of the incident signals. In (69) we have $M=22$, the constant 10 is for the unit conversion from Neper $(N p)$ to decibel $(d B)$ and $D$ stands for the wave propagation distance just before the arrival of reflected echoes at the probes.

The attenuation coefficient is numerically evaluated in the ten samples only in the polycrystal "ref", as it is difficult to record coherent echo signals in the polycrystal "strong" due to high levels of backscattered noises (see Figure 17(b)). Figure 18 presents comparisons between the numerical measures by two solvers "cV_sDG" and "tDG" either for one sample or for all the ten samples by plotting

measures. The numerical measures are in quite good agreement. Up to $11 \mathrm{kHz}$ the attenuation level calculated by the solver "cV_sDG" is slightly higher than the one calculated by the solver "tDG". The attenuation coefficient calculated by the solver "cR_sDG" is also plotted in Figure 18 (a) and shows poor agreement with the other two solvers.

On the other hand, backscattered noise levels are numerically measured in the time domain for a given frequency $f_{0}$ by calculating normalized root-meansquare (rms) noise levels $N_{r m s}\left(f_{0} ; t\right)$ in the following way [25]27]:

$$
N_{r m s}\left(f_{0} ; t\right)=\frac{\sqrt{\frac{1}{10 \times M} \sum_{j=1}^{10 \times M}\left(v_{y}^{b}\left(f_{0} ; \boldsymbol{x}_{j}, t\right)-b\left(f_{0} ; t\right)\right)^{2}}}{E_{\max }\left(f_{0}\right)}
$$

with

$$
b\left(f_{0} ; t\right)=\frac{1}{10 \times M} \sum_{j=1}^{10 \times M} v_{y}^{b}\left(f_{0} ; \boldsymbol{x}_{j}, t\right)
$$

where $10 \times M$ means the average is taken over all probes and over all the ten samples. For a given frequency $f_{0}$, Equation $(70)$ defines in the time domain the rms positional average of the difference between the noise signal $v_{y}^{b}\left(f_{0} ; \boldsymbol{x}_{j}, t\right)$ and the mean noise level $b\left(f_{0} ; t\right)$. The noise signal $v_{y}^{b}\left(f_{0} ; \boldsymbol{x}_{j}, t\right)$ is calculated by applying a frequency filtering to the noise signal $v_{y}^{b}\left(\boldsymbol{x}_{j}, t\right)=v_{y}\left(\boldsymbol{x}_{j}, t\right)-v_{y}^{r e f}\left(\boldsymbol{x}_{j}, t\right)$ recorded at the $j$ th receiver $\boldsymbol{x}_{j}$. The mean noise level $b\left(f_{0} ; t\right)$ would be zero if the total number of measures $10 \times M$, equal to 220 in the present work, was sufficiently large. In this case, $N_{r m s}\left(f_{0} ; t\right)$ is identical to the standard deviation of the noise level, normalized by $E_{\max }\left(f_{0}\right)$ in order to eliminate the dependence of the noise level on the incident power. $E_{\max }\left(f_{0}\right)$ is taken equal to one half 950 of the peak-to-peak amplitude of the incident signals for the frequency $f_{0}$, as proposed in [26].

A comparison between different solvers of the normalized rms noise levels $N_{r m s}\left(f_{0} ; t\right)$ in the time domain for the ten samples of the polycrystal "ref" is 
presented in Figure 19 for two frequencies $f_{0}=5$ and $10 \mathrm{MHz}$. Backscattered noise levels evaluated by the three solvers "cV_sDG", "cR_sDG" and "tDG" are in quite good agreement. However, it can be noticed that, for $f_{0}=5 \mathrm{MHz}$, both space dG solvers underestimate noise levels with respect to the levels calculated by the solver "tDG". On the other hand, Figure 19 (b) shows that, for $f_{0}=$ $10 \mathrm{MHz}$, the noise level calculated by the solver "cR_sDG" begins to exceed the

960 levels obtained by the other two solvers, which probably indicates the beginning of a instability problem. This observation is consistent with the previously obtained conclusions.

A comparison between the two solvers "cV_sDG" and "tDG" of the normalized rms noise levels $N_{r m s}\left(f_{0} ; t\right)$ in the time domain for the ten samples of the polycrystal "strong" is presented in Figure 20 for two frequencies $f_{0}=$ 5 and $10 \mathrm{MHz}$. The gaps between the measures obtained by the two solvers are greater and the backscattered noise levels evaluated by the solver "cV_sDG" is much lower for both frequencies.

In summary, the conclusions made in the $1 \mathrm{D}$ and $2 \mathrm{D}$ cases with only one physical interface are confirmed. The uncoupled numerical flux does not work at all whatever the degree of inhomogeneity. The space dG solver using the Reuss average coupled flux encounters instability problems when the degree of inhomogeneity is high and gives bad measure of the attenuation coefficient even in the case of low degree of inhomogeneity. The space dG solver using the Voigt 975 average coupled flux gives results in good agreement with the reference solvers in the case of low degree of inhomogeneity both for the attenuation coefficient and for the backscattered noise levels. However, it underestimates the noise levels and the underestimation becomes more important with the increase of the degree of inhomogeneity.

\section{Conclusions}

A unified variational framework for the space discontinuous Galerkin method for elastic wave propagation in anisotropic and piecewise homogeneous media was presented in multidimensional cases and for a general Hooke tensor. Its compact, wave oriented and intrinsic tensorial form allows a better understand-

985 ing of the physical meaning of the terms involved in the variational formulation, especially concerning the development of numerical fluxes.

Three upwind numerical fluxes were proposed and analyzed. As the numerical flux proposed by Käser et al., they solve only approximately the Riemann problem defined at physical interfaces in piecewise homogeneous media with discontinuous material properties. Numerical investigations were made firstly in $1 \mathrm{D}$ and $2 \mathrm{D}$ cases with only one physical interface and secondly in $2 \mathrm{D}$ cases of polycrystalline materials. As an important conclusion of the present work, it was shown that the necessary condition for a space $d G$ solver of having a stability behavior independent from the degree of inhomogeneity is that its nu-

995 merical flux solves exactly the Riemann problem. The uncoupled upwind flux does not work at all even if the degree of inhomogeneity is low. Concerning the two coupled fluxes, as an interesting result, the Reuss average has been shown 
to be less pertinent than the Voigt average in the present context. Indeed, the space dG solver using the Reuss average coupled flux encounters instability problems with the increase of the degree of inhomogeneity and gives bad evaluation of the attenuation level in polycrystalline materials. The space dG solver using the Voigt average coupled flux was shown to be interesting, as it is easy to implement and gives good evaluation in terms of both attenuation and backscattered noise levels when the degree of inhomogeneity is low. However,

1005 its underestimation of noise levels increases with the degree of inhomogeneity, it is necessary to develop more appropriate upwind numerical fluxes in the most general case of anisotropic and piecewise homogeneous media.

The proposed unified variational framework greatly facilitates this development. Indeed, in our current work several upwind numerical fluxes are derived using exact solutions of the Riemann problem. Part of this work, namely the upwind fluxes in the 1D case, were presented herein. Their comparison with the proposed simplified numerical fluxes leads to a better understanding of the characteristics of these latter. As one of the main results of the present work, we believe that our analysis of interface conditions is the key to design appropri-

1015 ate numerical fluxes. Indeed, the importance of defining a flux operator, which is coherent with the mechanical interface conditions regardless mathematically equivalent forms of the first-order hyperbolic wave equations, has been highlighted.

\section{Acknowledgments}

${ }_{1020}$ This work was granted by the ANR (Agence Nationale de la Recherche) - MAPIE project, ANR-13-MONU-002. Computations were performed using HPC resources from the computing center of CentraleSupélec supported by CentraleSupélec and CNRS (Centre National de la Recherche Scientifique).

\section{References}

1025 [1] B. Cockburn, G. Karniadakis, C.-W. Shu, (Eds.), Discontinuous Galerkin Methods. Theory, Computation and Applications, Lecture Notes in Computational Science and Engineering, volume 11, ISBN-13:978-3-64264098-8, Springer-Verlag, 2000.

[2] M. Käser, M. Dumbser, An arbitrary high-order discontinuous Galerkin method for elastic waves on unstructured meshes I: The two-dimensional isotropic case with external source terms, Geophysical Journal International 166 (2) (2006) 855-877.

[3] M. Dumbser, M. Käser, An arbitrary high-order discontinuous Galerkin method for elastic waves on unstructured meshes II: The three-dimensional isotropic case, Geophysical Journal International 167 (1) (2006) 319-336. 
[4] J. S. Hesthaven, T. Warburton, Nodal Discontinuous Galerkin Methods: Algorithms, Analysis, and Applications, 1st Edition, Springer Publishing Company, Incorporated, 2007.

[5] J. de la Puente, M. Käser, M. Dumbser, H. Igel, An arbitrary high order discontinuous Galerkin method for elastic waves on unstructured meshes IV: Anisotropy, Geophysical Journal International 169 (3) (2007) 12101228.

[6] V. Etienne, E. Chaljub, J. Virieux, N. Glinsky, An hp-adaptive discontinuous Galerkin finite-element method for 3-D elastic wave modelling, Geophysical Journal International 183 (1) (2010) 941-962.

[7] L. Wilcox, G. Stadler, C. Burstedde, O. Ghattas, A high-order discontinuous Galerkin method for wave propagation through coupled elastic-acoustic media, Journal of computational Physics 229 (2010) 9373-9396.

[8] M. Stanglmeiera, N. Nguyena, J. Perairea, B. Cockburn, An explicit hybridizable discontinuous Galerkin method for the acoustic wave equation, Computer Methods in Applied Mechanics and Engineering 300 (2010) 748769 .

[9] R. LeVeque, Finite Volume Methods for Hyperbolic Problems, Cambridge Texts in Applied Mathematics, Cambridge University Press, 2002.

[10] V. A. Titarev, E. F. Toro, Ader: Arbitrary high order Godunov approach, J. Sci. Comput. 17 (1-4) (2002) 609-618.

[11] P. Moczo, J. Kristek, V. Vavrycuk, A. R.J., L. Halada, 3D heterogeneous staggered-grid finite-difference modeling of seismic motion with volume harmonic and arithmetic averaging of elastic moduli and densities, Bulletin of the Seismological Society of America 92 (8) (2002) 3042-3066.

[12] E. Toro, Riemann Solvers and Numerical Methods for Fluid Dynamics: A Practical Introduction. 2nd Edition, Springer-Verlag Berlin Heidelberg, 1999.

[13] D. Arnold, F. Brezzi, B. Cockburn, L. Marini, Unified analysis of discontinuous galerkin methods for elliptic problems, SIAM J. Numer. Anal. 39 (5) (2002) 1749-1779.

[14] B. Cockburn, C.-W. Shu, Runge-Kutta Discontinuous Galerkin Methods for Convection-Dominated Problems, J. Sci. Comput. 16 (3) (2002) 173261.

[15] T. J. Hughes, G. Hulbert, Space-time finite element methods for elastodynamics: Formulations and error estimates, Computer Methods in Applied Mechanics and Engineering 66 (1988) 339-363. 
[16] C. Johnson, Discontinuous Galerkin finite element methods for second order hyperbolic problems, Computer Methods in Applied Mechanics and Engeneering 107 (1993) 117-129.

[17] M. Li, N. Wiberg, Implementation and adaptivity of space-time finite element method for structural dynamics, Computer Methods in Applied Mechanics and Engineering 156 (1998) 211-229.

[18] B. Tie, D. Aubry, Adaptive time discontinuous Galerkin method for numerical modeling of wave propagation in shell and 3D structures, European Journal of computational Mechanics 15 (6) (2006) 729-757.

[19] J.-M. Leclère, Parallel and adaptive FE modeling of elastic wave propagation in structures (Modélisation parallèle de la propagation d'ondes dans les structures par éléments finis adaptatifs), Ph.D. thesis, École Centrale de Paris (in French) (2001).

[20] A. Grédé, Numerical modeling of pyrotechnic shock wave propagation in Ariane5's structures (Modélisation des chocs d'origine pyrotechnique dans les structures d'Ariane5 : Développement de modèles de propagation et d'outils de modélisation), Ph.D. thesis, École Centrale de Paris (in French) (2009).

[21] T. Ekevid, M. X. Li, N. Wiberg, Adaptive FEA of wave propagation induced by high-speed trains, Computers and Structures 79 (2001) 26932704 .

[22] E. Priolo, J. Carcione, G. Seriani, Numerical simulation of interface waves by high-order spectral modeling techniques, Journal of the Acoustical Society of America 95(3) (1994) 681-693.

[23] F. E. Stanke, G. S. Kino, A unified theory for elastic wave-propagation in polycrystalline materials, Journal of the Acoustical Society of America 75(3) (1984) 665-681.

[24] B. Tie, D. Solas, J. Thébault, C. Rey, T. Baudin, A. Mouronval, Numerical modeling of ultrasound propagation in polycrystalline materials (Modélisation numérique de la propagation des ultrasons dans des milieux polycristallins), in: 10th French Congress of Acoustics (CFA2010), Lyon, France, Avril 2010, p. 6 pages (in french).

[25] X. Bai, Finite element modeling of ultrasonic wave propagation in polycrystalline materials, Ph.D. thesis, CentraleSupélec (2017). URL https://tel.archives-ouvertes .fr/tel-01483701/document

[26] F. Margetan, R. Thompson, I. Yalda-Mooshabad, Backscattered microstructural noise in ultrasonic toneburst inspections, Journal of Nondestructive Evaluation 13(3) (1994) 111-136. 
[27] X. Bai, B. Tie, J.-H. Schmitt, D. Aubry, Finite element modeling of grain size effects on the ultrasonic microstructural noise backscattering in polycrystalline materials, Ultrasonics 87 (2018) 182-202.

\section{Appendix A1}

The well-known rotational invariance property of the isotropic elastic wave problem (6) or 9) is proved hereafter in the tridimensional case.

Lemma A1.1. In the isotropic case, the two matrices, $\left[A_{\boldsymbol{e}_{1}}\right]$ expressed in the global basis $\left(\boldsymbol{e}_{1}, \boldsymbol{e}_{2}, \boldsymbol{e}_{3}\right),\left[A_{\boldsymbol{n}}\right]$ expressed in the local basis $\left(\boldsymbol{n}, \boldsymbol{t}_{1}, \boldsymbol{t}_{2}\right)$ are identical.

Proof. In the global basis $\left(\boldsymbol{e}_{1}, \boldsymbol{e}_{2}, \boldsymbol{e}_{3}\right)$, the term of the matrix $\left[A_{\boldsymbol{e}_{1}}\right]_{\gamma, \alpha \beta ; k, i j}$ can be calculated as follows:

$$
\begin{aligned}
{\left[A_{\boldsymbol{e}_{1}}\right]_{\gamma, \alpha \beta ; k, i j} } & =\left(\begin{array}{c}
\boldsymbol{e}_{\gamma} \\
\boldsymbol{e}_{\alpha} \otimes_{s} \boldsymbol{e}_{\beta}
\end{array}\right) \cdot \boldsymbol{A}_{\boldsymbol{e}_{1}}\left(\begin{array}{c}
\boldsymbol{e}_{k} \\
\boldsymbol{e}_{i} \otimes_{s} \boldsymbol{e}_{j}
\end{array}\right) \\
& =\left(\begin{array}{c}
\boldsymbol{e}_{\gamma} \\
\boldsymbol{e}_{\alpha} \otimes_{s} \boldsymbol{e}_{\beta}
\end{array}\right) \cdot\left(\begin{array}{c}
-\rho^{-1}\left(\boldsymbol{e}_{i} \otimes_{s} \boldsymbol{e}_{j}\right) \cdot \boldsymbol{e}_{1} \\
-\boldsymbol{C}:\left(\boldsymbol{e}_{1} \otimes_{s} \boldsymbol{e}_{k}\right)
\end{array}\right) \\
& =-\frac{1}{2 \rho}\left(\delta_{1 i} \delta_{\gamma j}+\delta_{1 j} \delta_{\gamma i}\right)-C_{\alpha \beta 1 k}
\end{aligned}
$$

with $C_{\alpha \beta 1 k}=\left(\boldsymbol{e}_{\alpha} \otimes_{s} \boldsymbol{e}_{\beta}\right): \boldsymbol{C}:\left(\boldsymbol{e}_{1} \otimes_{s} \boldsymbol{e}_{k}\right)$ due to the definition of $\boldsymbol{A}_{\boldsymbol{e}_{1}}$ (8) and the symmetry properties of the elastic tensor $C$.

In the local basis $\left(\boldsymbol{b}_{1}=\boldsymbol{n}, \boldsymbol{b}_{2}=\boldsymbol{t}_{1}, \boldsymbol{b}_{3}=\boldsymbol{t}_{2}\right)$, the term of the matrix $\left[A_{\boldsymbol{n}}\right]_{\gamma, \alpha \beta ; k, i j}$ can be calculated as follows:

$$
\begin{aligned}
{\left[A_{n}\right]_{\gamma, \alpha \beta ; k, i j} } & =\left(\begin{array}{c}
\boldsymbol{b}_{\gamma} \\
\boldsymbol{b}_{\alpha} \otimes_{s} \boldsymbol{b}_{\beta}
\end{array}\right) \cdot \boldsymbol{A}_{\boldsymbol{n}}\left(\begin{array}{c}
\boldsymbol{b}_{k} \\
\boldsymbol{b}_{i} \otimes_{s} \boldsymbol{b}_{j}
\end{array}\right) \\
& =\left(\begin{array}{c}
\boldsymbol{b}_{\gamma} \\
\boldsymbol{b}_{\alpha} \otimes_{s} \boldsymbol{b}_{\beta}
\end{array}\right) \cdot\left(\begin{array}{c}
-\rho^{-1}\left(\boldsymbol{b}_{i} \otimes_{s} \boldsymbol{b}_{j}\right) \cdot \boldsymbol{n} \\
-\boldsymbol{C}:\left(\boldsymbol{n} \otimes_{s} \boldsymbol{b}_{k}\right)
\end{array}\right) \\
& =-\frac{1}{2 \rho}\left(\delta_{1 i} \delta_{\gamma j}+\delta_{1 j} \delta_{\gamma i}\right)-C_{\alpha \beta 1 k}^{\text {local }}
\end{aligned}
$$

with $C_{\alpha \beta 1 k}^{\text {local }}=\left(\boldsymbol{b}_{\alpha} \otimes_{s} \boldsymbol{b}_{\beta}\right): \boldsymbol{C}:\left(\boldsymbol{n} \otimes_{s} \boldsymbol{b}_{k}\right)$. In the isotropic case, $C_{i j k l}$ is independent from the basis, for $\forall i, j, k, l$, i.e. $C_{\alpha \beta 1 k}=C_{\alpha \beta 1 k}^{l o c a l}$, which finally leads to $\left[A_{\boldsymbol{e}_{1}}\right]_{\gamma, \alpha \beta ; k, i j}=\left[A_{\boldsymbol{n}}\right]_{\gamma, \alpha \beta ; k, i j}$.

\section{Appendix A2}

The expressions (39) of the solutions $\left(\alpha, \alpha^{\prime}\right)$ of the Riemann problem (37) is proven hereafter.

By adding the three equations of (37), i.e. Eqn. $37 \mathrm{a}+$ Eqn. $37 \mathrm{~b}+$ Eqn. $37 \mathrm{c}$, we get:

$$
\boldsymbol{A}_{\boldsymbol{n}}\left(\boldsymbol{U}_{h}\right)-\alpha \lambda_{\boldsymbol{n}}^{-} \boldsymbol{R}_{\boldsymbol{n}}^{-}+\boldsymbol{A}_{\boldsymbol{n}^{\prime}}^{\prime}\left(\boldsymbol{U}_{h}^{\prime}\right)-\alpha^{\prime} \lambda_{\boldsymbol{n}^{\prime}}^{-\prime} \boldsymbol{R}_{\boldsymbol{n}^{\prime}}^{-{ }^{\prime}}=\mathbf{0}
$$


According to the definition of the Jacobian operator 10, , the eigenmodes 18 and the definition (15) and the property (14) of the Christoffel tensor $\boldsymbol{\Gamma}_{\boldsymbol{n}}$, we obtain the following equations by considering separately $\wp_{\text {vect }}$ (Eqn.(74)) and $\boldsymbol{n} \cdot \wp_{\text {tens }}($ Eqn. 74$)$ :

$$
\begin{gathered}
-\frac{\boldsymbol{\sigma}_{h} \cdot \boldsymbol{n}}{\rho}-\frac{\boldsymbol{\sigma}_{h}^{\prime} \cdot \boldsymbol{n}^{\prime}}{\rho^{\prime}}=\alpha \lambda_{\boldsymbol{n}}^{-} \frac{\gamma_{\boldsymbol{n}}}{\sqrt{2}}+\alpha^{\prime} \lambda_{\boldsymbol{n}^{\prime}}^{-\frac{\gamma_{\boldsymbol{n}^{\prime}}^{\prime}}{\sqrt{2}}} \\
-\rho \boldsymbol{\Gamma}_{\boldsymbol{n}} \cdot \boldsymbol{v}_{h}+\rho^{\prime} \boldsymbol{\Gamma}_{\boldsymbol{n}^{\prime}}^{\prime} \cdot \boldsymbol{v}_{h}^{\prime}=-\alpha \rho \lambda_{\boldsymbol{n}}^{-2} \frac{\gamma_{\boldsymbol{n}}}{\sqrt{2}}+\alpha^{\prime} \rho^{\prime} \lambda_{\boldsymbol{n}^{\prime}}^{-{ }^{\prime}} \frac{\gamma_{\boldsymbol{n}^{\prime}}^{\prime}}{\sqrt{2}}
\end{gathered}
$$

Then, to obtain the expressions for $\alpha$ and $\alpha^{\prime}$ given in 39 , the following manipulations of 75 are made:

- For $\alpha, C_{z}^{\prime} \frac{\gamma_{n}}{\sqrt{2}} \cdot\left(-\frac{\rho^{\prime} \lambda_{n^{\prime}}^{-^{\prime}}}{\rho \lambda_{n}^{-2}}\right.$ Eqn. $\left.75 \mathrm{a}\right)+\frac{1}{\rho \lambda_{n}^{-2}}$ Eqn. $\left.75 \mathrm{~b}\right)$;

- For $\alpha^{\prime}, C_{z} \frac{\gamma_{\boldsymbol{n}^{\prime}}^{\prime}}{\sqrt{2}} \cdot\left(-\frac{\rho \lambda_{n}^{-}}{\rho^{\prime} \lambda_{\boldsymbol{n}^{\prime}}^{-\prime 2}}\right.$ Eqn. $75 \mathrm{a}-\frac{1}{\rho^{\prime} \lambda_{\boldsymbol{n}^{\prime}}^{\mathbf{\prime}^{\prime 2}}}$ Eqn. $\left.75 \mathrm{~b}\right)$.

\section{Appendix A3}

The expressions $(50)$ of the solutions $\left(\tilde{\alpha}, \tilde{\alpha}^{\prime}\right)$ of the Riemann problem 45 is proven hereafter.

By adding the three equations of 45 , i.e. Eqn. $45 \mathrm{a}+$ Eqn. $45 \mathrm{~b}+$ Eqn. $45 \mathrm{c}$, we get:

$$
\tilde{\boldsymbol{A}}_{\boldsymbol{n}}\left(\boldsymbol{U}_{h}\right)-\tilde{\alpha} \lambda_{\boldsymbol{n}}^{-} \boldsymbol{M}\left(\boldsymbol{R}_{\boldsymbol{n}}^{-}\right)+\tilde{\boldsymbol{A}}_{\boldsymbol{n}^{\prime}}^{\prime}\left(\boldsymbol{U}_{h}^{\prime}\right)-\tilde{\alpha}^{\prime} \lambda_{\boldsymbol{n}^{\prime}}^{-^{\prime}} \boldsymbol{M}^{\prime}\left(\boldsymbol{R}_{\boldsymbol{n}^{\prime}}^{-{ }^{\prime}}\right)=\mathbf{0}
$$

According to the definition of $\tilde{\boldsymbol{A}}_{\boldsymbol{n}}(47)$, the eigenmodes $(18)$, the definition (15) and the property (14) of the Christoffel tensor $\boldsymbol{\Gamma}_{\boldsymbol{n}}$ and the relation (48), we obtain the following equations by applying $\wp_{v e c t}(\cdot)$ and $\wp_{\text {tens }}(\cdot)$ on Eqn. (76):

$$
\begin{gathered}
-\boldsymbol{\sigma}_{h} \cdot \boldsymbol{n}-\boldsymbol{\sigma}_{h}^{\prime} \cdot \boldsymbol{n}^{\prime}=\tilde{\alpha} \rho \lambda_{\boldsymbol{n}}^{-} \frac{\gamma_{\boldsymbol{n}}}{\sqrt{2}}+\tilde{\alpha}^{\prime} \rho^{\prime} \lambda_{\boldsymbol{n}^{\prime}}^{-{ }^{\prime}} \frac{\gamma_{\boldsymbol{n}^{\prime}}^{\prime}}{\sqrt{2}} \\
-\boldsymbol{n} \otimes_{s} \boldsymbol{v}_{h}-\boldsymbol{n}^{\prime} \otimes_{s} \boldsymbol{v}_{h}^{\prime}=-\tilde{\alpha} \boldsymbol{n} \otimes_{s} \frac{\gamma_{\boldsymbol{n}}}{\sqrt{2}}-\tilde{\alpha}^{\prime} \boldsymbol{n}^{\prime} \otimes_{s} \frac{\gamma_{\boldsymbol{n}^{\prime}}^{\prime}}{\sqrt{2}}
\end{gathered}
$$

1145 Then, to obtain the expressions for $\tilde{\alpha}$ and $\tilde{\alpha}^{\prime}$ given in $(50)$, the following manipulations of (77) are made:

- For $\tilde{\alpha}, C_{z}\left(-\frac{1}{\rho^{\prime} \lambda_{\boldsymbol{n}^{\prime}}^{-\prime}} \frac{\gamma_{\boldsymbol{n}}}{\sqrt{2}} \cdot\right.$ Eqn. $\left.77 \mathrm{a}\right)+\frac{1}{\rho \lambda_{\boldsymbol{n}}^{-2}}\left(\boldsymbol{n} \otimes_{s} \frac{\gamma_{\boldsymbol{n}}}{\sqrt{2}}\right): \boldsymbol{C}:$ Eqn. $\left.77 \mathrm{~b}\right)$;

- For $\left\{\tilde{\alpha}_{k}^{\prime}\right\}, C_{z}^{\prime}\left(-\frac{1}{\rho \lambda_{n}^{-}} \frac{\gamma_{\boldsymbol{n}^{\prime}}^{\prime}}{\sqrt{2}} \cdot\right.$ Eqn. $(77 \mathrm{a})+\frac{1}{\rho^{\prime} \lambda_{\boldsymbol{n}^{\prime}, k}^{\mathbf{\prime}^{\prime} 2}}\left(\boldsymbol{n}^{\prime} \otimes_{s} \frac{\gamma_{\boldsymbol{n}^{\prime}}^{\prime}}{\sqrt{2}}\right): \boldsymbol{C}^{\prime}$ : Eqn. $(77 \mathrm{~b})$. 


\section{Appendix A4}

${ }_{1150}$ The elementary mass, stiffness, flux stiffness matrices and the elementary loading vector are detailed as follows:

$$
\begin{aligned}
M_{p q}^{E E} & =\left(\boldsymbol{\Psi}_{p}^{E}, \boldsymbol{\Psi}_{q}^{E}\right)_{E} \\
K_{p q}^{E E} & =\left(\boldsymbol{\Psi}_{p}^{E}, \boldsymbol{A}^{\partial_{x}}\left(\boldsymbol{\Psi}_{q}^{E}\right)\right)_{E} \\
K_{p q}^{f, E E} & =-<\lambda_{\boldsymbol{n}, k}^{-} \boldsymbol{R}_{\boldsymbol{n}, k}^{-} \cdot \boldsymbol{\Psi}_{p}^{E}, \boldsymbol{L}_{\boldsymbol{n}, k}^{-} \cdot \boldsymbol{\Psi}_{q}^{E}>_{\partial E_{i n t}}+<\boldsymbol{\Pi}_{v}\left(\boldsymbol{\Psi}_{p}^{E}\right), \rho^{-1} \boldsymbol{\Pi}_{\sigma}\left(\boldsymbol{\Psi}_{q}^{E}\right) \cdot \boldsymbol{n}>_{\partial E \cap \partial \Omega_{N}} \\
K_{p q}^{f, E E^{\prime}} & =-<\lambda_{\boldsymbol{n}^{\prime}, k}^{\prime+} \boldsymbol{R}_{\boldsymbol{n}^{\prime}, k}^{+} \cdot \boldsymbol{\Psi}_{p}^{E}, \boldsymbol{L}_{\boldsymbol{n}^{\prime}, k}^{\prime+} \cdot \boldsymbol{\Psi}_{q}^{E^{\prime}}>_{\partial E_{i n t}} \\
G_{p}^{E} & =<\boldsymbol{\Pi}_{v}\left(\boldsymbol{\Psi}_{p}^{E}\right), \rho^{-1} \boldsymbol{g}>_{\partial E \cap \partial \Omega_{N}}-<\boldsymbol{\Pi}_{\sigma}\left(\boldsymbol{\Psi}_{p}^{E}\right), \boldsymbol{C}:\left(\boldsymbol{n} \otimes_{s} \partial_{t} \tilde{\boldsymbol{u}}_{D}\right)>_{\partial E \cap \partial \Omega_{D}}
\end{aligned}
$$

with

$$
\boldsymbol{\Pi}_{v}(\boldsymbol{W})=\boldsymbol{\Pi}_{v}\left(\begin{array}{c}
\boldsymbol{w} \\
\boldsymbol{\tau}
\end{array}\right)=\boldsymbol{w}, \boldsymbol{\Pi}_{\sigma}(\boldsymbol{W})=\boldsymbol{\Pi}_{\sigma}\left(\begin{array}{c}
\boldsymbol{w} \\
\boldsymbol{\tau}
\end{array}\right)=\boldsymbol{\tau}
$$




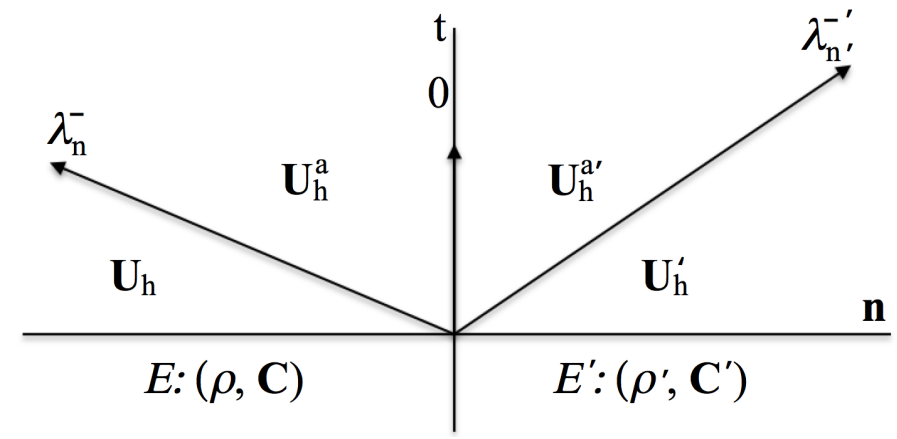

Figure 1: Sketch illustrating the Rankine-Hugoniot jump conditions in the Riemann problem in the $1 \mathrm{D}$ case

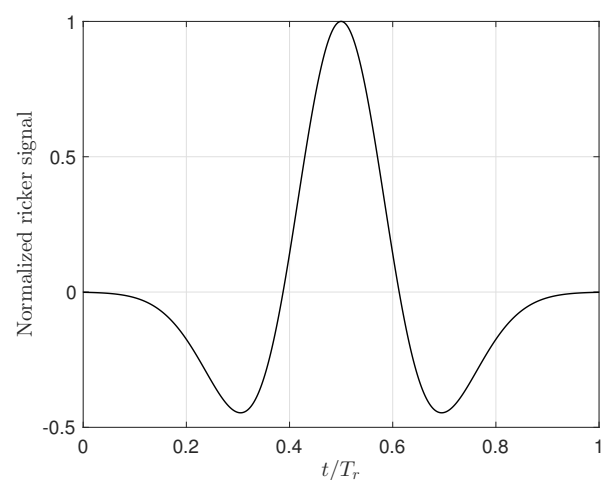

(a)

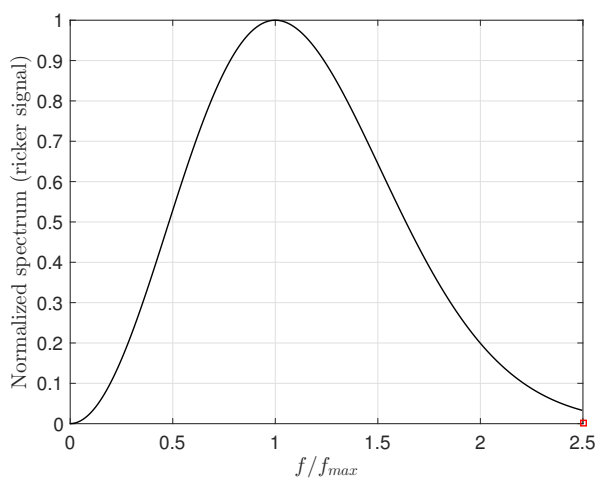

(b)

Figure 2: (a) Normalized ricker signal and (b) its normalized spectrum with $t_{d}=T_{r} / 2$

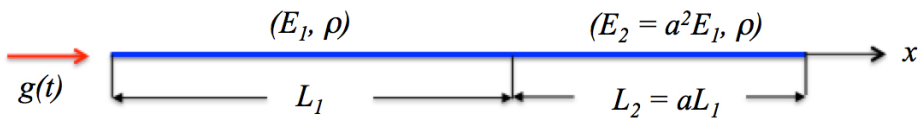

(a)

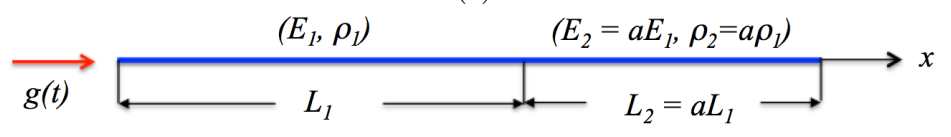

(b)

Figure 3: 1D elastic rod with one physical interface. (a) Case 1 with discontinuous Young's modulus; (b) Case 2 with discontinuous Young's modulus and density 


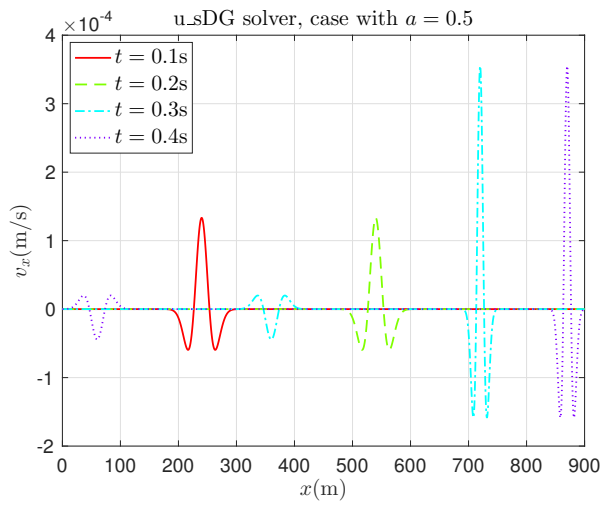

(a)

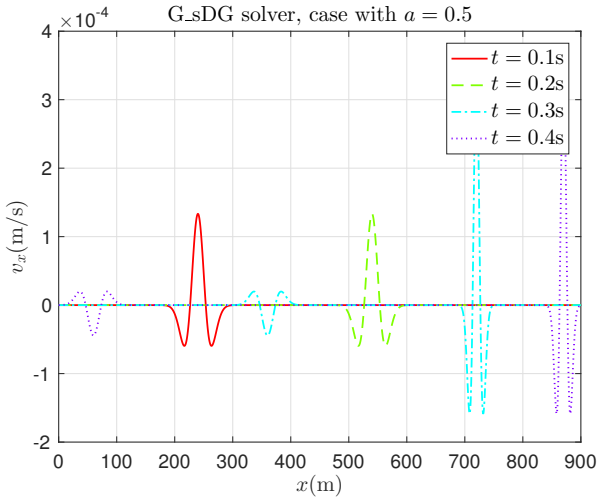

(b)

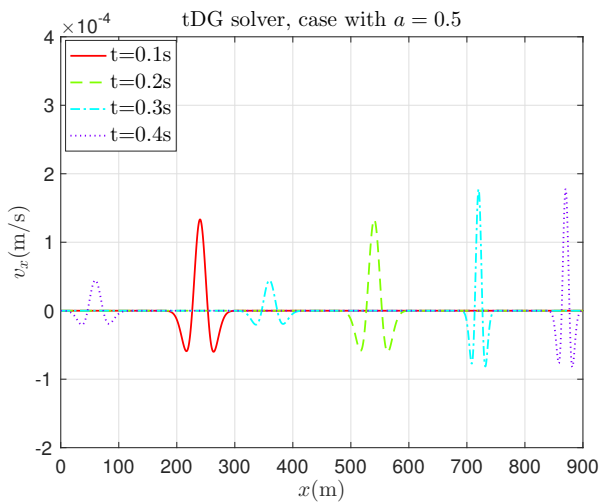

(c)

Figure 4: Longitudinal wave propagation in the studied elastic rod for Case 1 (Figure 3(a)) with $a=0.5$ calculated by the solvers (a) "u_sDG", (b) "G_sDG" and (c) "tDG" 


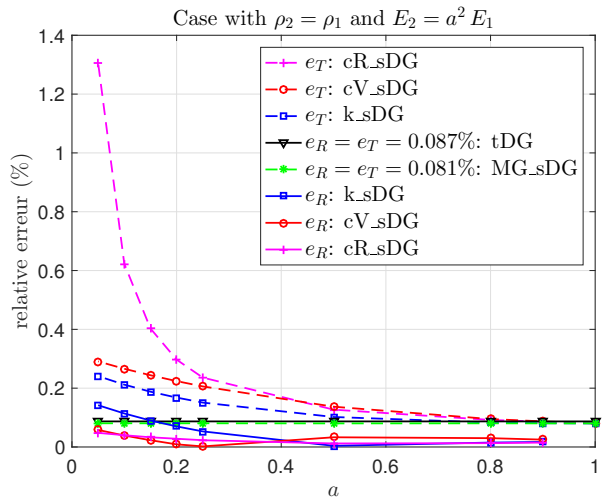

(a)

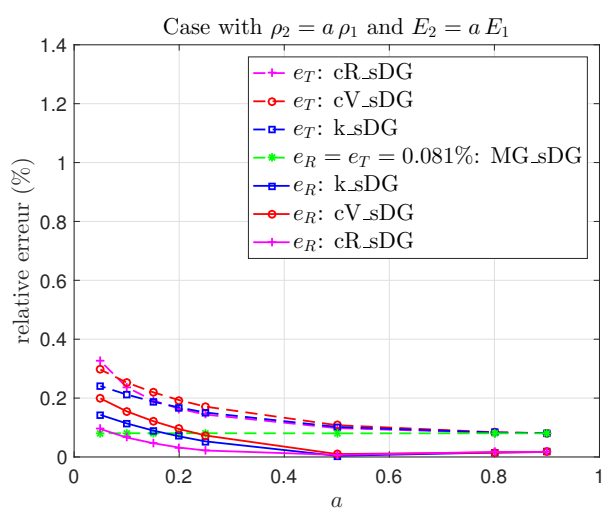

(b)

Figure 5: Evolution of relative errors in the reflection and transmission coefficients as a fonction of the degree of inhomogeneity $a$. (a) Case 1 with $\rho_{2}=\rho_{1}$ and $E_{2}=a^{2} E_{1}$; (b) Case 2 with $\rho_{2}=a \rho_{1}$ and $E_{2}=a E_{1}$ 

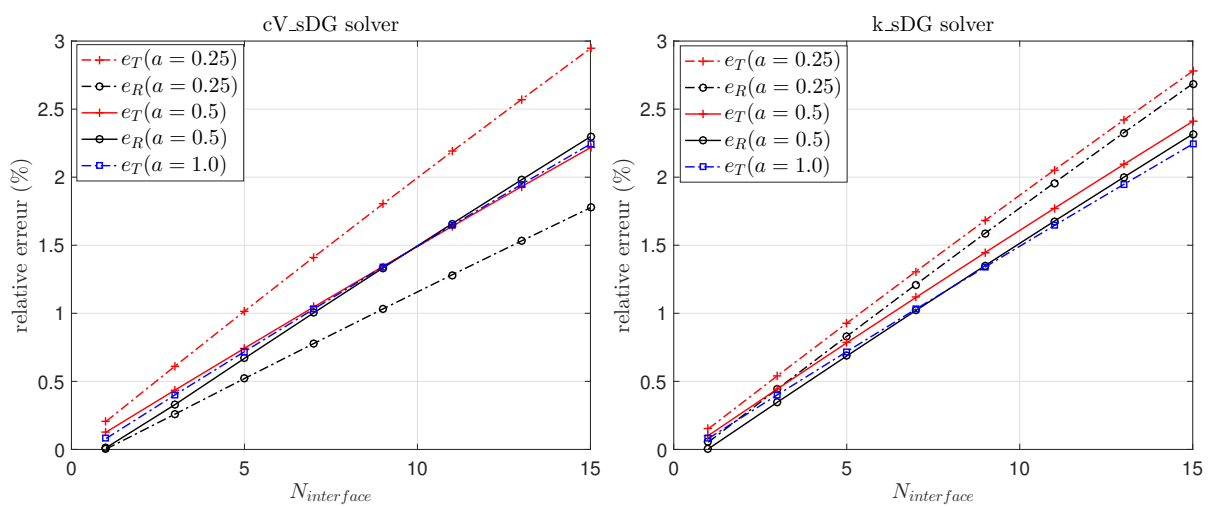

(a)
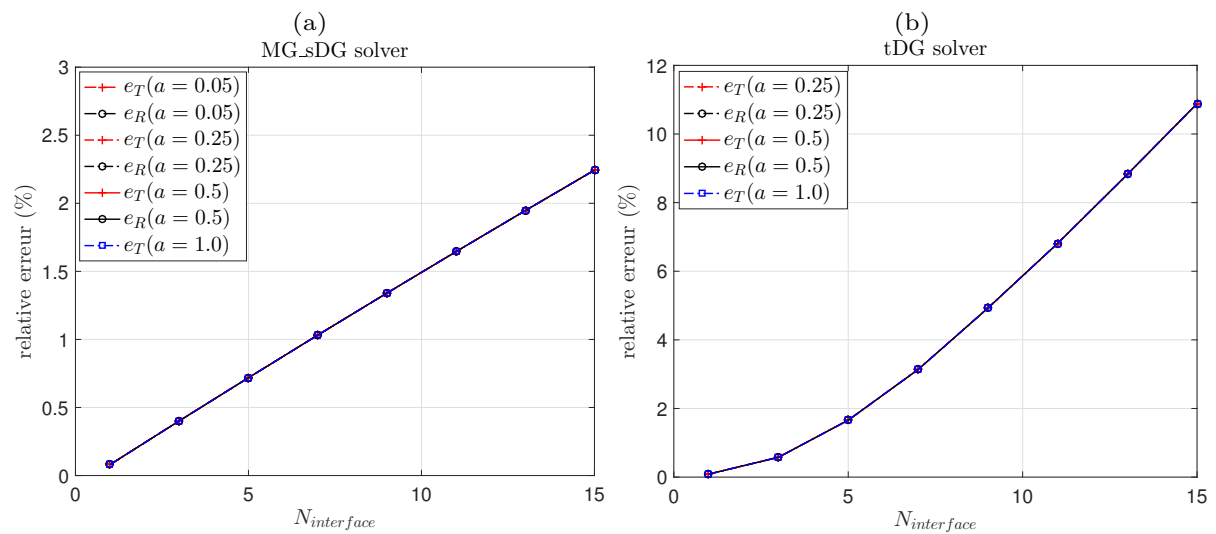

(c)

(d)

Figure 6: Evolution of relative errors in the reflection and transmission coefficients as a fonction of the number of interface crossing for the solvers (a) "cV_sDG", (b) "k_sDG", (c) "MG_sDG" and (d) "tDG"

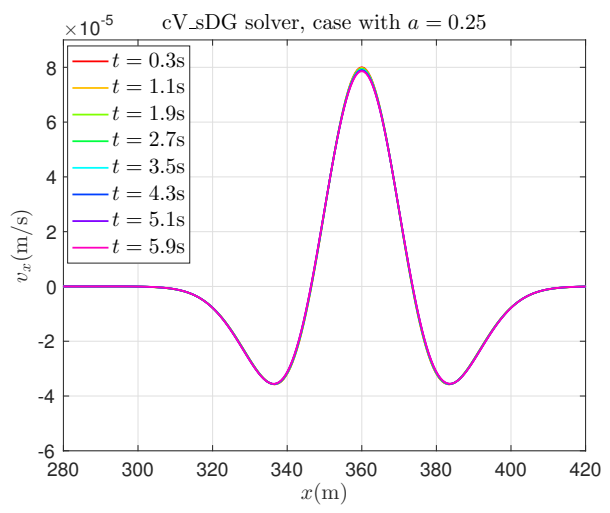

(a)

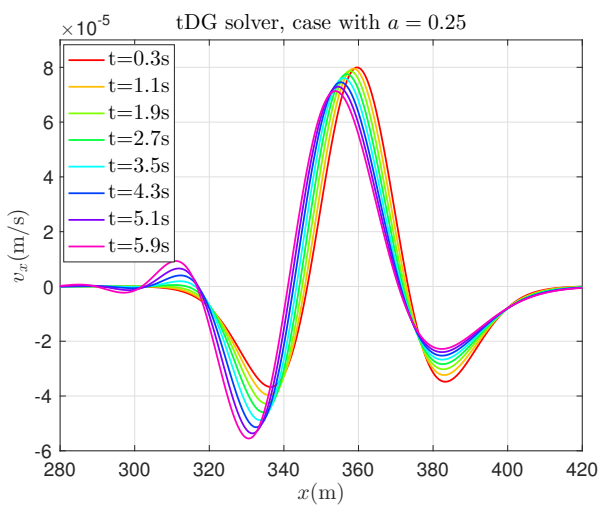

(b)

Figure 7: Reflected wave fronts calculated at different instants by the solvers (a) "cV_sDG" and (b) "tDG" in the case of $a=0.25$ 


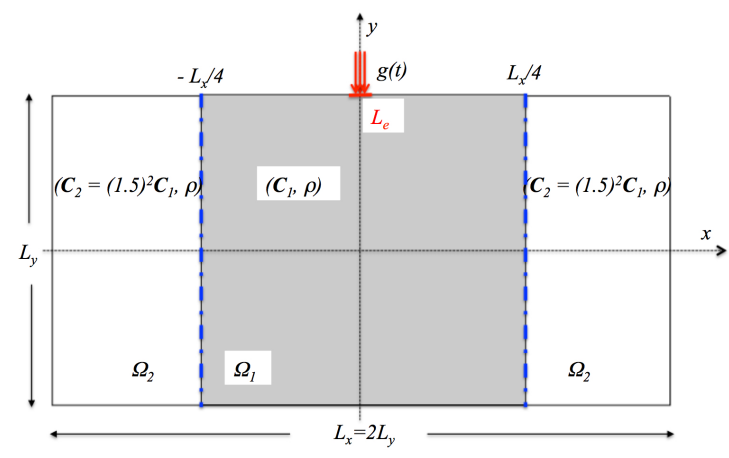

Figure 8: 2D rectangular elastic domain with two vertical physical interfaces 

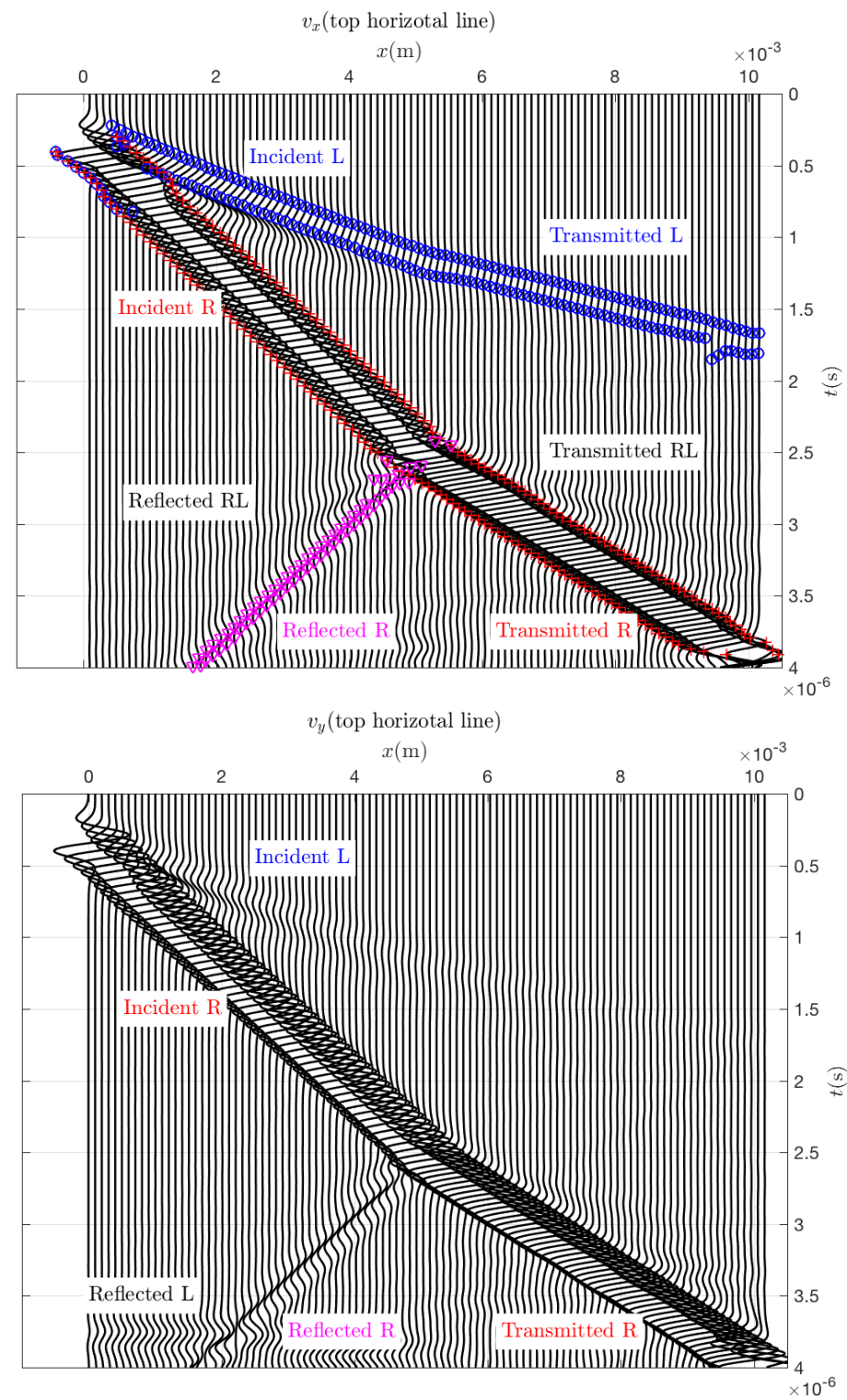

Figure 9: 2D bimaterial problem. Seismograms calculated by the solver "cV_sDG" along the top horizontal boundary of the studied domain for the velocity components $v_{x}$ and $v_{y}$. The symbols $\mathrm{L}, \mathrm{R}$ and $\mathrm{RL}$ refer to longitudinal, transverse and from $\mathrm{R}$ to $\mathrm{L}$ converted waves respectively 

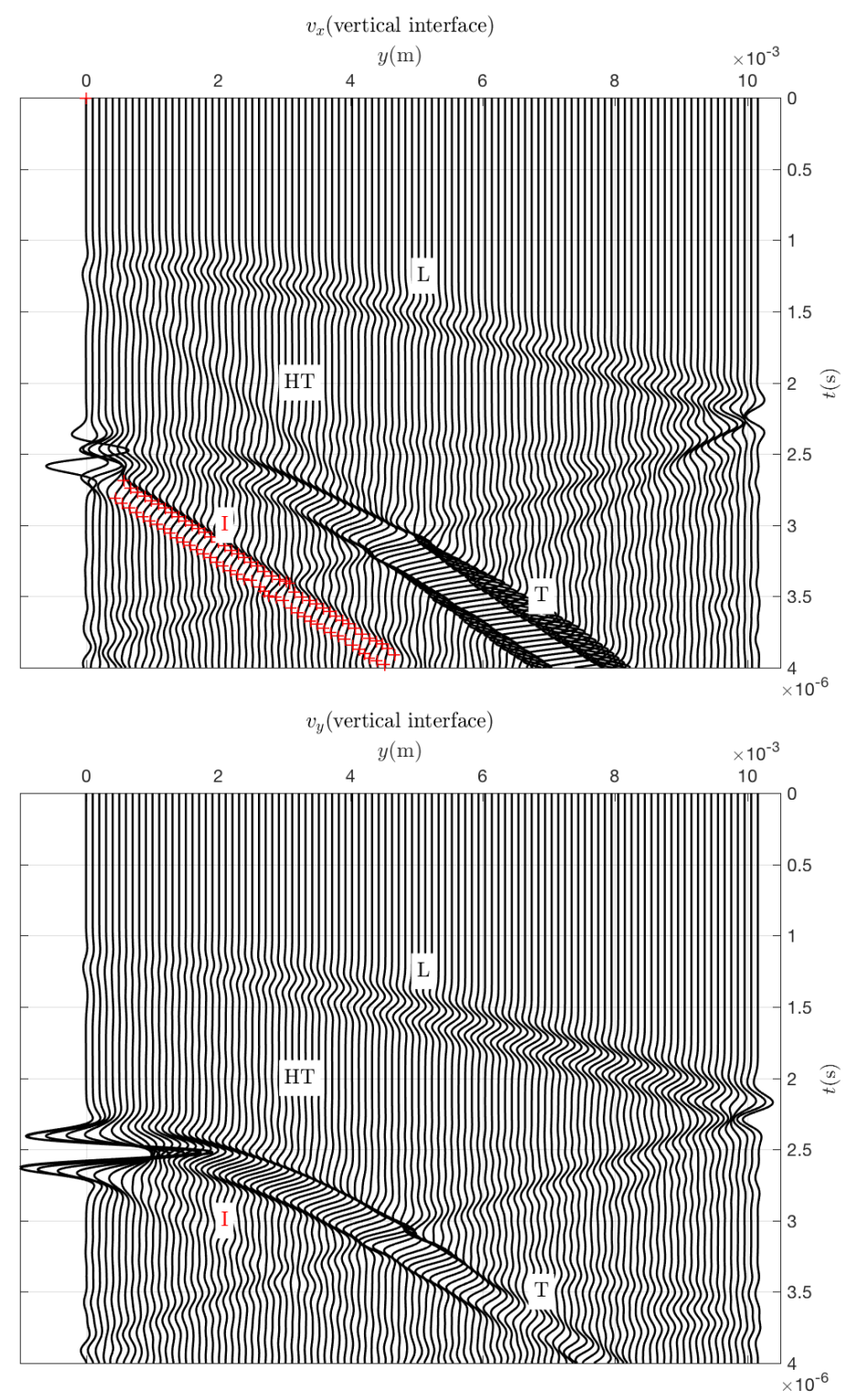

Figure 10: 2D bimaterial problem. Seismograms calculated by the solver "cV_sDG" along the vertical physical interface in the studied domain for the velocity components $v_{x}$ and $v_{y}$. The symbols L, T, HT and I to longitudinal, transverse, transverse head and interface waves, respectively. 


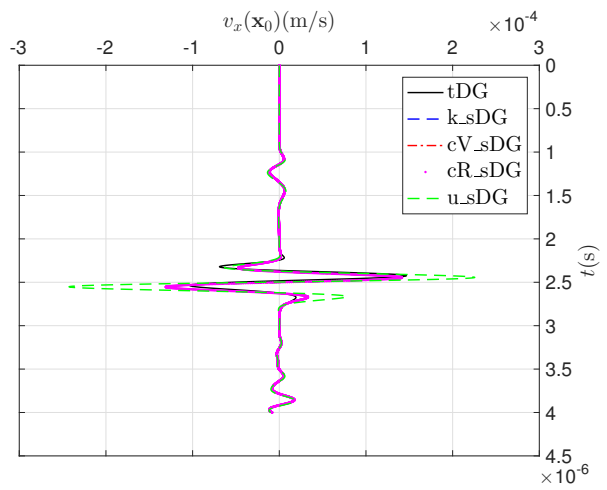

(a)

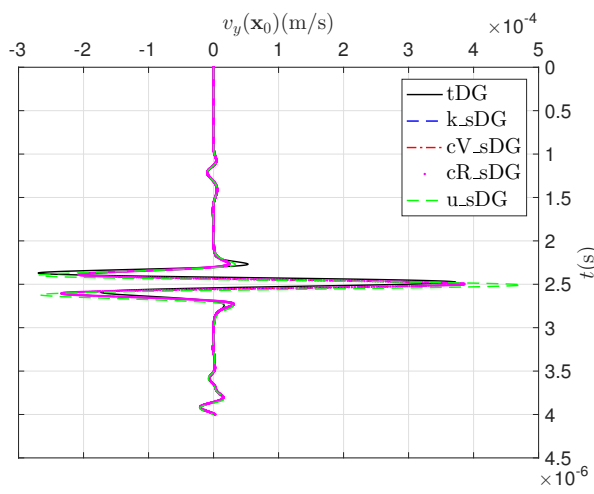

(b)

Figure 11: 2D bimaterial problem. Comparison between the solvers "k_sDG", "u_sDG", "cV_sDG" and "tDG" of the time evolution of the velocity components (a) $v_{x}$ and (b) $v_{y}$ recorded at a point near the physical interface and on the top boundary $\boldsymbol{x}_{0}=(5.0,5.08) \mathrm{mm}$

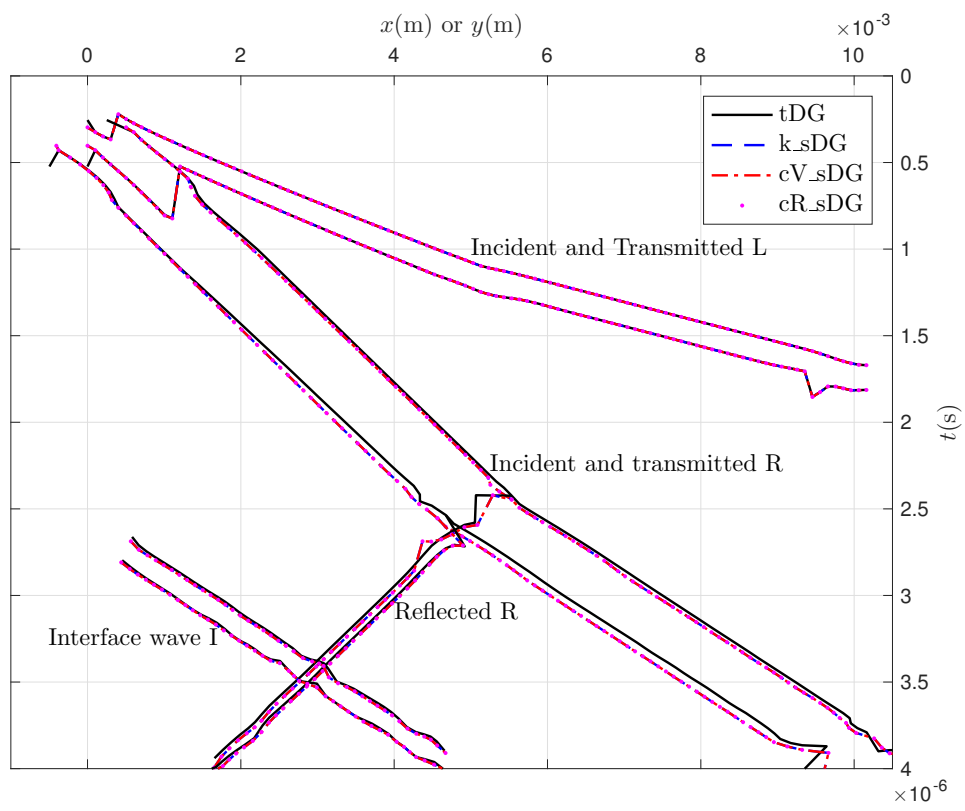

Figure 12: 2D bimaterial problem. Comparison of different waves fronts: (1) incident and transmitted $\mathrm{L}$ waves, (2) incident and transmitted $\mathrm{R}$ waves, (3) reflected $\mathrm{R}$ waves and (4) interface waves, captured by the three solvers "tDG", "k_sDG", "cV_sDG" and "cR_sDG" 


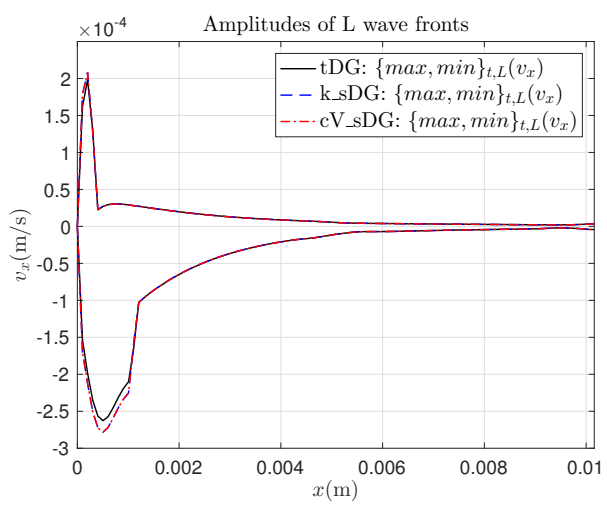

(a)

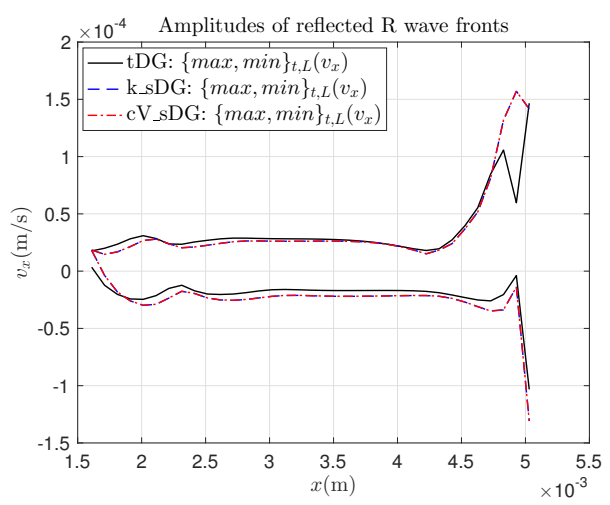

(c)

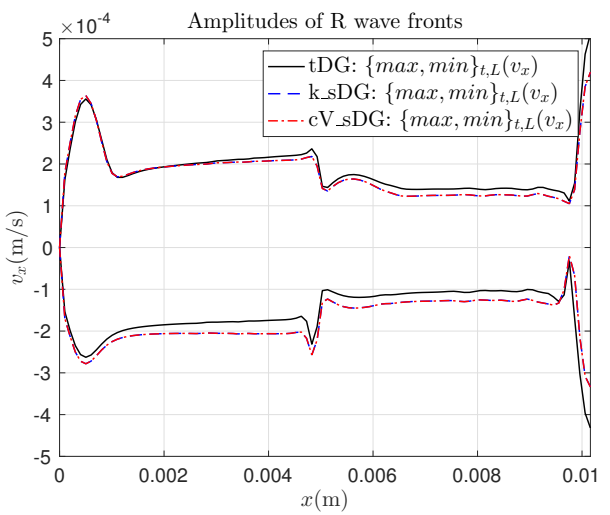

(b)

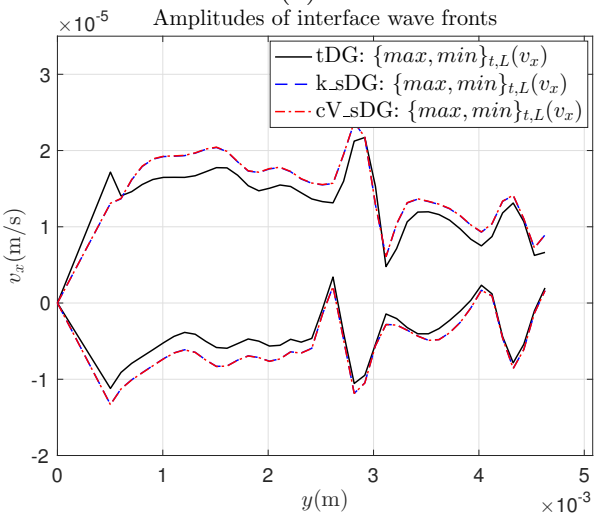

(d)

Figure 13: 2D bimaterial problem, comparison between the solvers "k_sDG", "cV_sDG" and "tDG". Evolution of maximum and minimum amplitudes of the (a) incident and transmitted $\mathrm{L}$, (b) incident and transmitted $\mathrm{R}$, (c) reflected $\mathrm{R}$ and (d) interface wave fronts during their propagation 


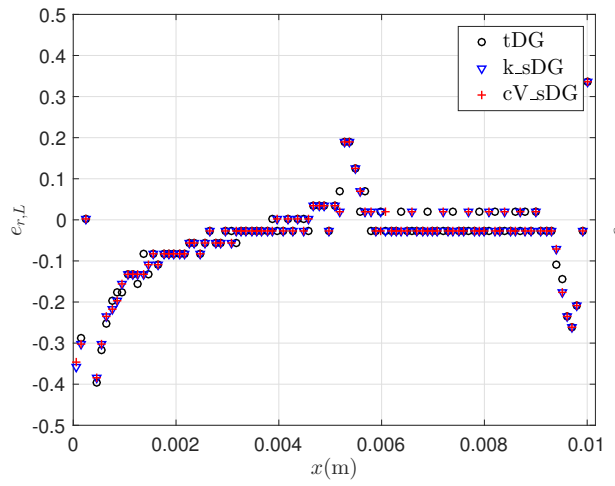

(a)

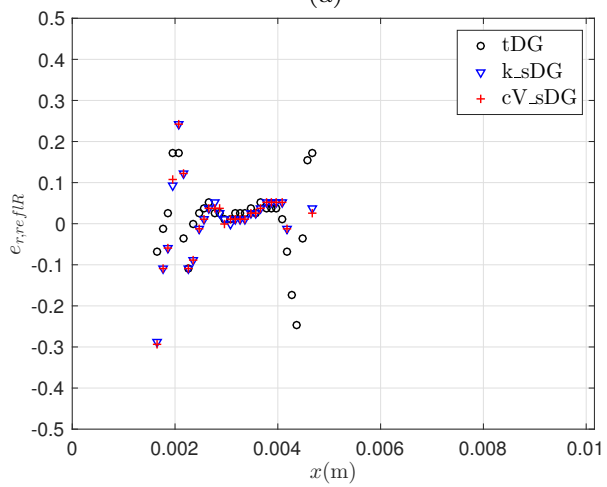

(c)

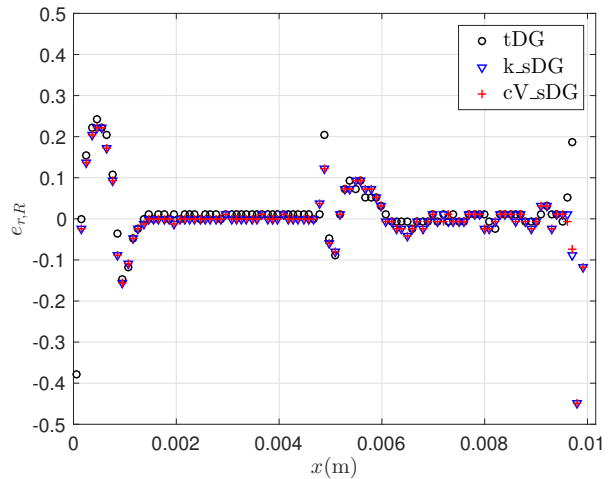

(b)

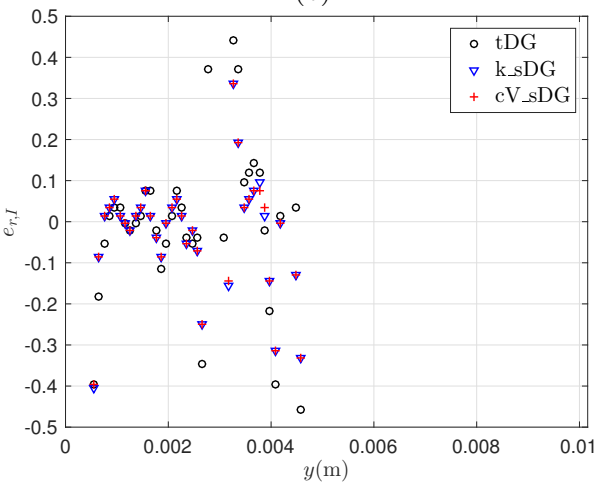

(d)

Figure 14: 2D bimaterial problem, comparison between the solvers "k_sDG", "cV_sDG" and "tDG". Relative errors in phase velocities obtained by the different solvers for the (a) incident and transmitted L, (b) incident and transmitted R, (c) reflected R and (d) interface waves 


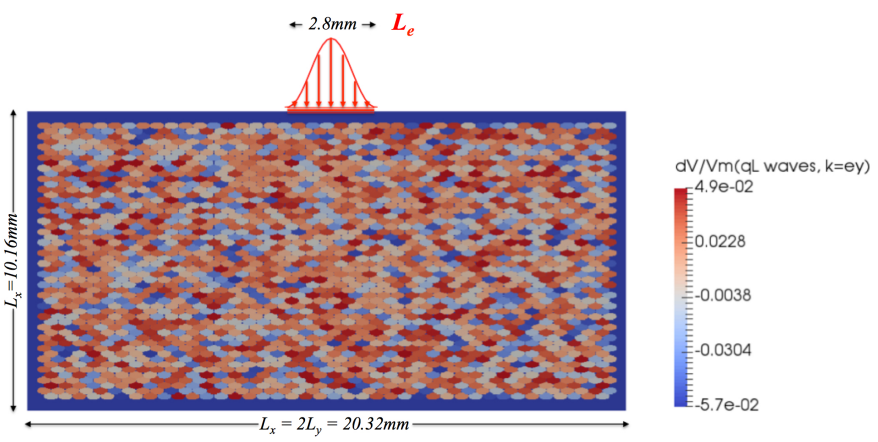

(a)
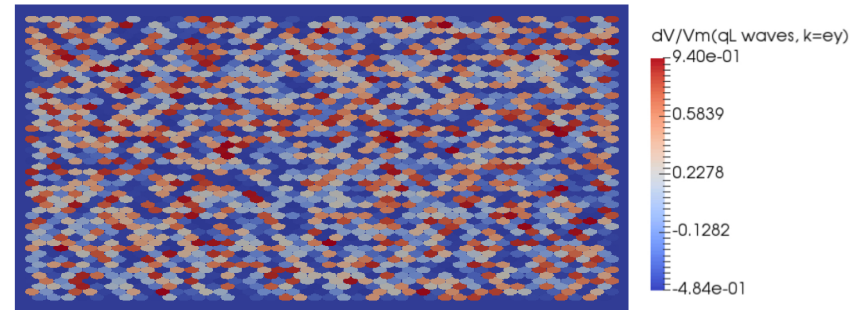

(b)

Figure 15: (a) FE model of a single-phase polycrystal composed of elliptic grains with pressure loading applied on the emitter segment $L_{e}$ with a Gaussian distribution. Dispersions $d V / V_{m}$ in the phase wave velocity $c_{q L}(\boldsymbol{k})$ with $\boldsymbol{k}=\boldsymbol{e}_{y}$ for the polycrystals (a) "ref" and (b) "strong", $V_{m}$ being the averaged wave velocity $\left\langle c_{q L}(\boldsymbol{k})>_{\text {grains }}\right.$ over all grains and $d V=c_{q L}(\boldsymbol{k})-V_{m}$ for each grain

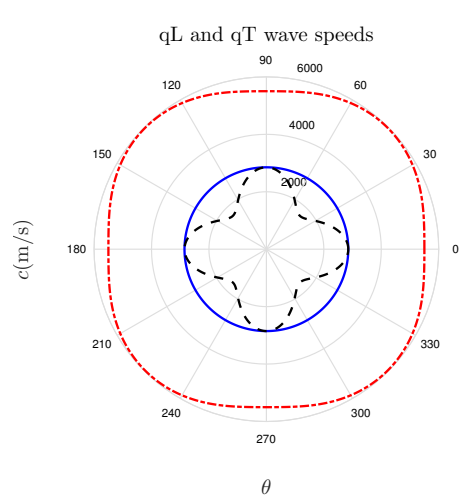

(a)

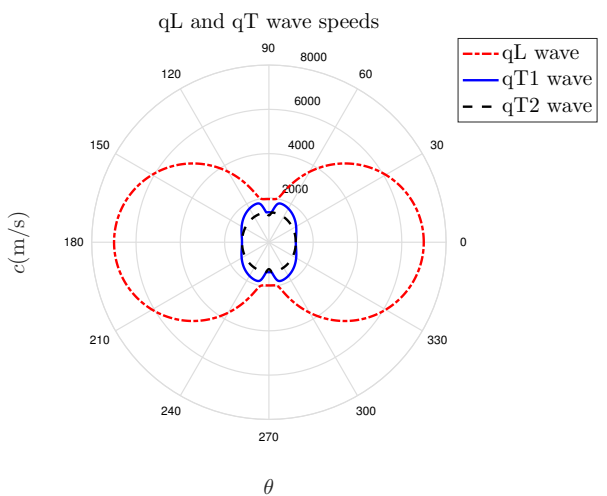

(b)

Figure 16: Wave velocities of the anisotropic materials (a) "ref" and (b) "strong" in the local material anisotropic plane $\left(\boldsymbol{a}_{1}, \boldsymbol{a}_{2}\right)$ defined by the two first principle axes of anisotropy 


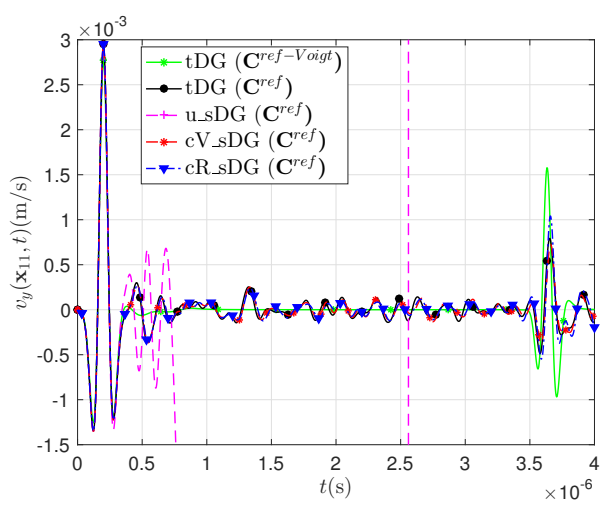

(a)

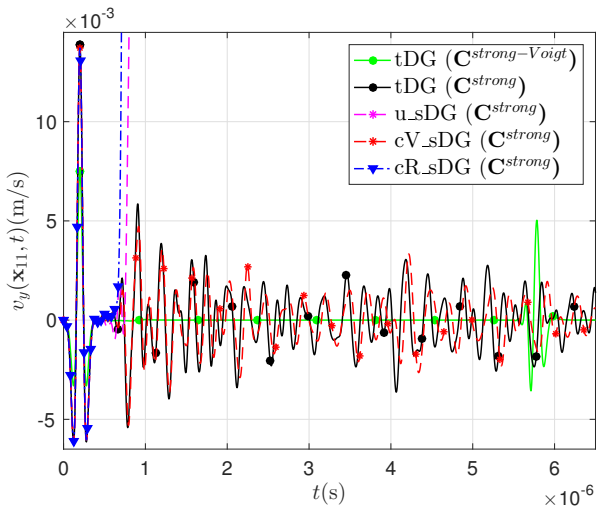

(b)

Figure 17: Velocity component $v_{y}$ at the middle of the emitter segment $L_{e}$ calculated by different solvers. (a) Instability phenomena encountered by the solver "u_sDG" in the case of the polycrystal "ref"; (b) Instability phenomena encountered by the solvers "u_sDG" and "cR_sDG" in the case of the polycrystal "strong"

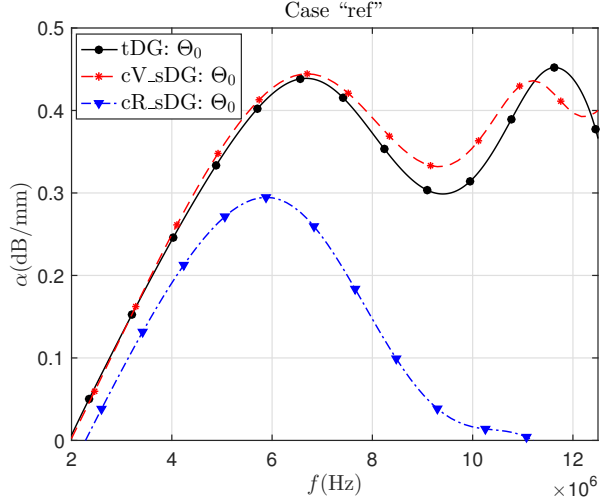

(a)

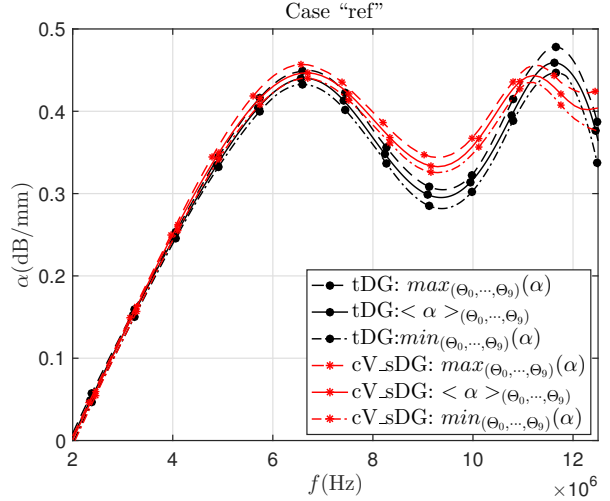

(b)

Figure 18: Comparison between different solvers of numerical measure of the attenuation coefficient $\alpha$ in the polycrystal "ref". (a) $\alpha$ measured in the sample $\Theta_{0}$; (b) Discrepancies of numerical measure with $\operatorname{mean}_{\Theta}(\alpha), \min _{\Theta}(\alpha)$ and $\max _{\Theta}(\alpha)$ denoting respectively the averaged numerical measures, the minimum and maximum bounds of numerical measures over the ten samples $\Theta_{0}-\Theta_{9}$ 


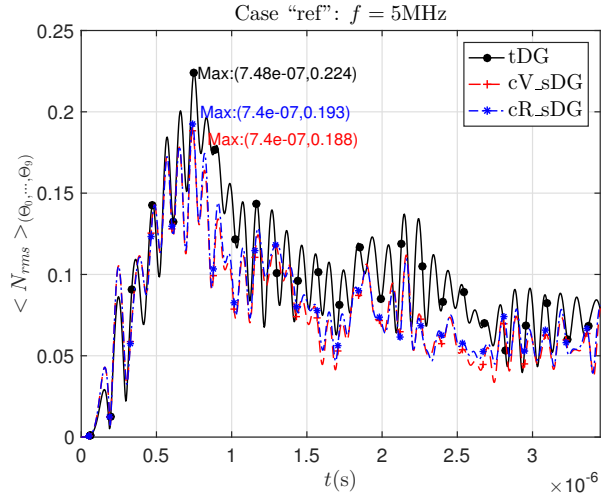

(a)

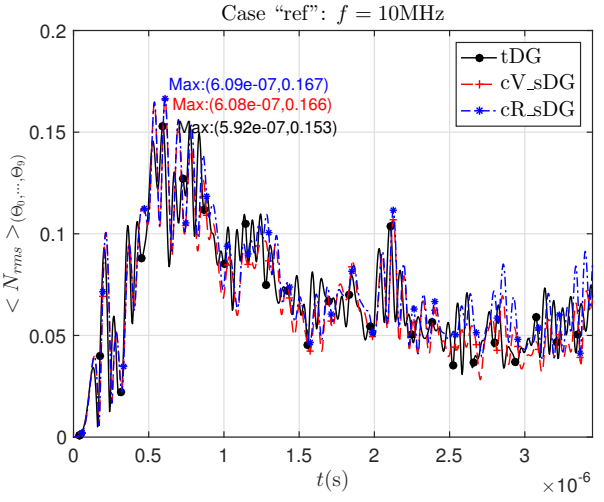

(b)

Figure 19: Comparison between different solvers of the normalized rms noise levels $N_{r m s}\left(f_{0} ; t\right)$ for the ten samples of the polycrystal "ref". (a) $f_{0}=5 \mathrm{MHz}$; (b) $f_{0}=10 \mathrm{MHz}$

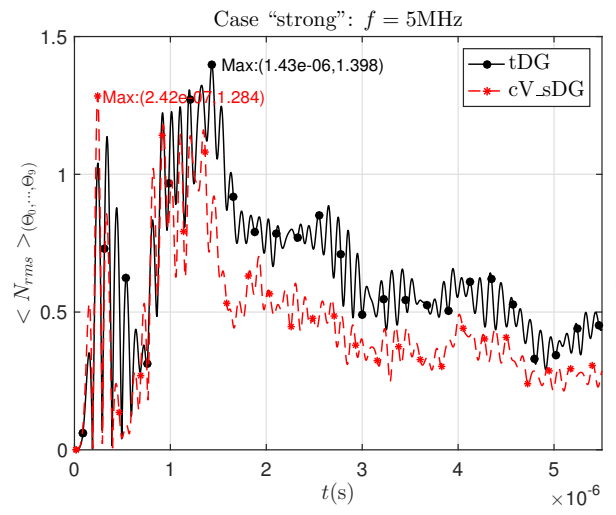

(a)

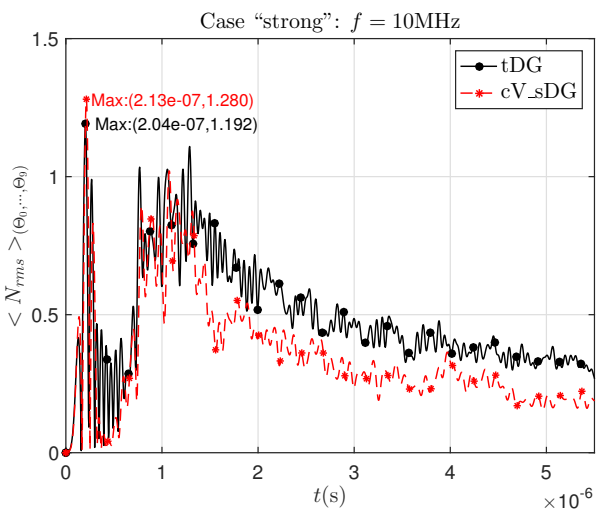

(b)

Figure 20: Comparison between different solvers of the normalized rms noise levels $N_{r m s}\left(f_{0} ; t\right)$ for the ten samples of the polycrystal "strong". (a) $f_{0}=5 \mathrm{MHz}$; (b) $f_{0}=10 \mathrm{MHz}$ 